\title{
The Ecological Role of a \\ Common Seastar (Patiriella spp.) Within Intertidal Cobble Fields
}

\author{
by \\ Shiree Palmer
}

\author{
A thesis submitted to the \\ Victoria University of Wellington \\ in partial fulfillment of the requirements for the degree of \\ Masters of Science in Marine Biology
}

School of Biological Sciences

Victoria University of Wellington

2010 


\section{Abstract}

Intertidal cobble habitats are complex three-dimensional marine environments that are understudied despite having unique species assemblages and ecological patterns. New Zealand's common cushion star, Patiriella spp., is found in a wide range of coastal habitats, including intertidal cobble fields. This seastar is an omnivore that feeds predominantly on crustose coralline algae and micro-organisms, but also supplements its diet by scavenging on carrion. Study on the adult ecology of Patiriella spp. is limited and this thesis aims to expand on the knowledge of this species and its role in intertidal cobble communities. First, field surveys were conducted within intertidal cobble fields in Wellington Harbour and on the Wellington South Coast to determine density, size and feeding habits of Patiriella spp. and the density of associated organisms. Patiriella spp. were abundant at all sites, with no significant difference in density between Wellington Harbour and Wellington South Coast; however, feeding rates and sizes were significantly higher on the South Coast. Distribution of Patiriella spp. on cobbles was negatively correlated with the distribution of chitons, suggesting possible competition between these animals. Second, scavenging behaviour was examined in field and laboratory experiments. Patiriella spp. were readily attracted to mussel carrion bait in the field; however, whelks were the numerically dominant taxon attracted to bait and may therefore compete with Patiriella spp. for this resource. Laboratory results showed that movement towards carrion may be indicative of hunger and Patiriella spp. from Wellington Harbour and the Wellington South Coast reacted similarly to carrion, suggesting similar, limited levels, of carrion supply within these regions. The occurrence of interspecific feeding competition was tested in the laboratory by examining growth and mortality in response to varying densities of Patiriella spp. and a locally abundant chiton, and possible competitor, Chiton glaucus. Intraspecific competition was also tested in response to varying densities of Patiriella spp., with and without carrion supplementation 
and during spring and winter. No inter- or intraspecific competition was found for crustose coralline algae and micro-organisms and this food resource appears not to be limited. However, Patiriella spp. supplemented on mussel carrion had significantly higher growth rates than non-supplemented treatments and this was greater at lower densities, suggesting intraspecific competition for carrion. Increases in Patiriella spp. size and pyloric caeca weight were only observed for treatments supplemented with carrion. Therefore, carrion appears important for growth and reproduction and intraspecific competition for this resource may impact population sizes. Consequently, fluctuations in carrion supply have the potential to change the distribution and abundance of Patiriella spp., leading to changes in community dynamics. This study has provided baseline information on Patiriella spp. populations within intertidal cobble fields in Wellington Harbour and on the Wellington South Coast and also improved knowledge of the feeding behaviours and competitive interactions of this seastar; therefore, contributing to understanding of the ecological role of Patiriella spp. within intertidal cobble communities. 


\section{Acknowledgements}

I would like to thank my supervisor Dr Nicole Phillips for her continual advice and encouragement from the moment I first considered doing my master's right though to its completion. She has given me invaluable advice on everything from thesis topic, through to methodology, statistics and write-up, while still allowing me scope to go in my own direction. Without her encouragement I would not have gotten this far.

Thanks to everyone in the Phillips-Shima lab group for their enthusiasm and advice and especially to Shane Geange and Anna Smith, for reviewing chapters and providing me with useful feedback. Also, thanks to our research assistant Jennifer Howe for assistance with lab experiments.

Thank you to the technical and administrative staff at VUCEL and SBS. Thanks to John van der Sman for being easy to approach, answering all my "silly" questions and providing the baskets for my lab experiments. Also, thanks to Patricia Stein, Mary Murray and Sandra Taylor.

Thanks to Kate Neill at NIWA for her advice on Patiriella spp. identification and to the Izard Scholarship for providing much appreciated funding.

I also want to thank my friends and family who provided me with endless support and encouragement. Special thanks goes to Helen Cranshaw for helping with animal collections and to my fiancé Darren Foster for his awesome emotional and financial support. Thanks to my mum Kathy Palmer for the phone chats which kept me going and also to my other mum Josie Palmer for looking after all my worldly possessions while I returned to study.

This work is dedicated to my dad Peter Palmer and my brother Adam Palmer. 


\section{Contents}

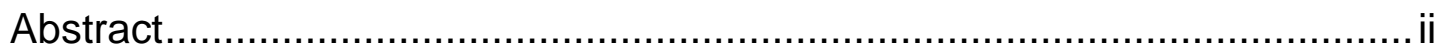

Acknowledgements ........................................................................... iv

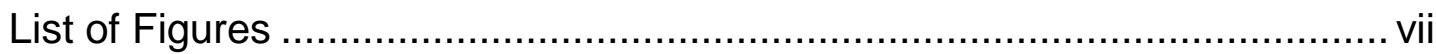

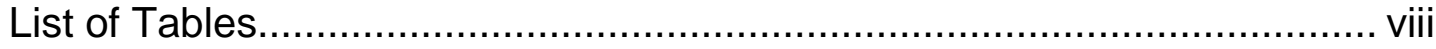

\section{Chapter 1: Introduction}

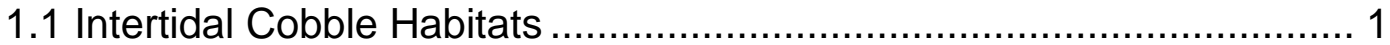

1.2 Biological Processes ................................................................. 3

1.3. Omnivores and Scavengers ……………….......................... 5

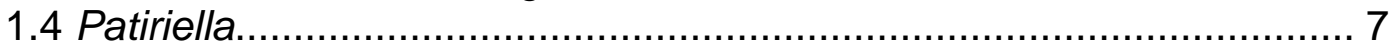

1.5 Wellington Harbour and South Coast .............................................. 9

1.6 Aims and Objectives............................................................... 11

Chapter 2: Density, Feeding Habits and Size of Patiriella spp. within Intertidal Cobble Fields

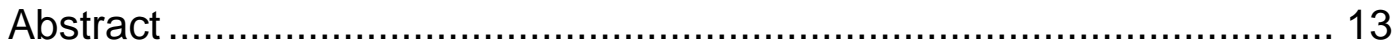

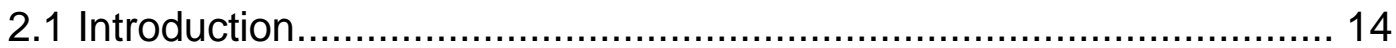

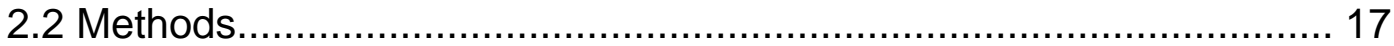

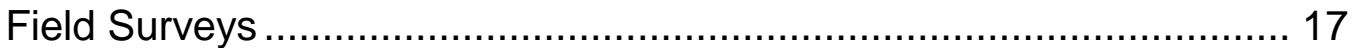

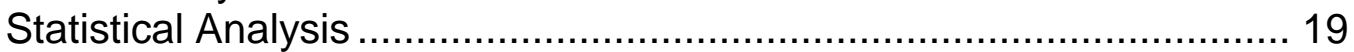

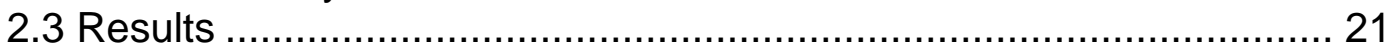

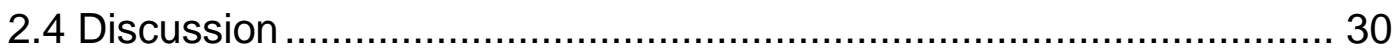

Chapter 3: Scavenging Behaviour of Patiriella spp.

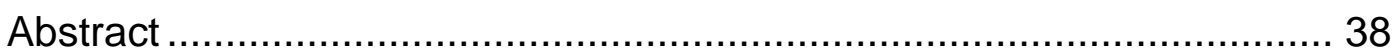

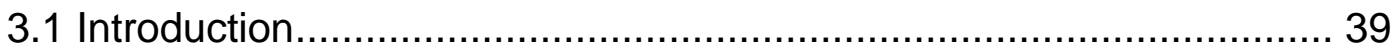

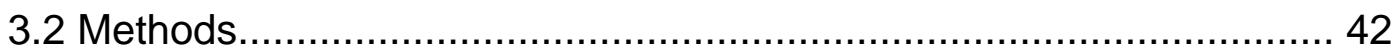

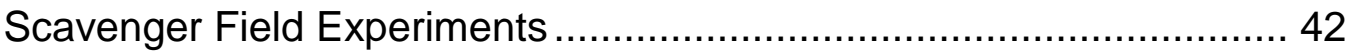

Scavenger Laboratory Experiments.............................................. 44

Statistical Analysis ............................................................... 46

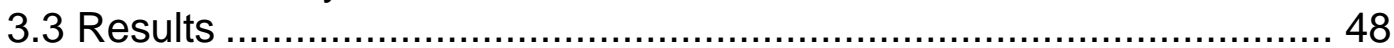

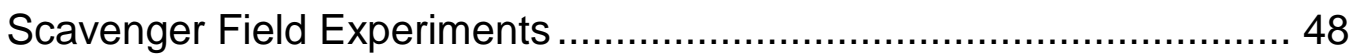

Scavenger Laboratory Experiments................................................ 49

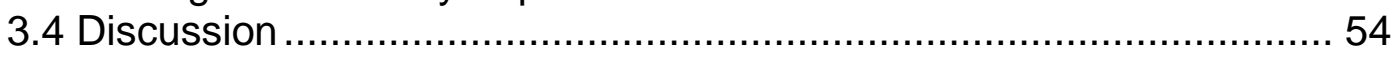


Chapter 4: Inter- and Intraspecific Feeding Competition in Patiriella spp.

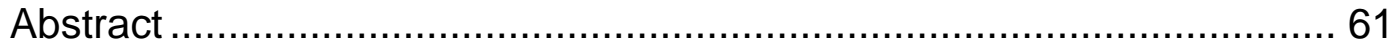

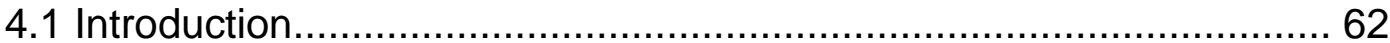

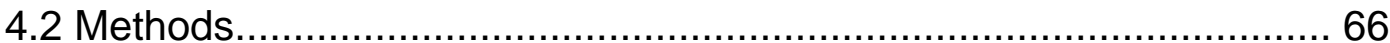

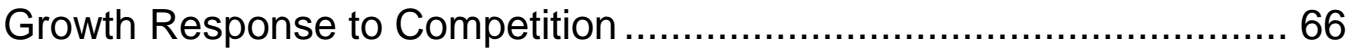

Reproductive Response to Competition ...........................................69

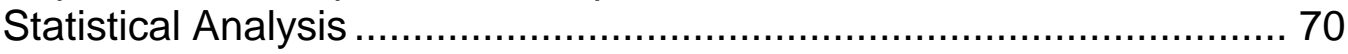

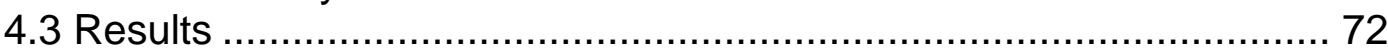

Growth Response to Competition ..................................................... 72

Reproductive Response to Competition .......................................... 76

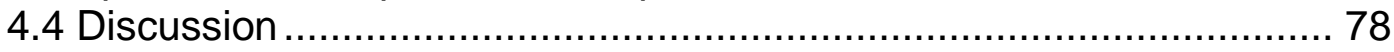

\section{Chapter 5: General Discussion}

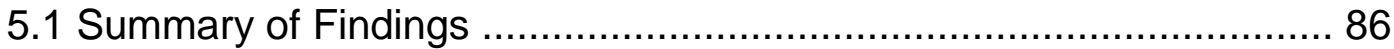

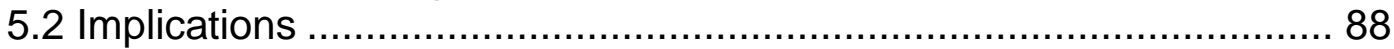

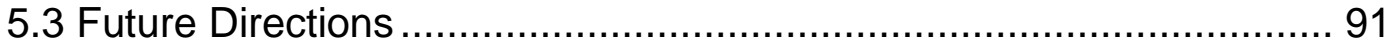

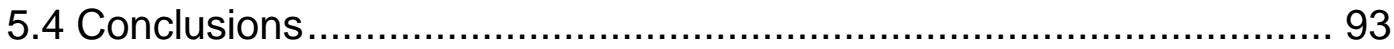

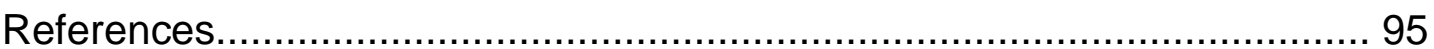




\section{List of Figures}

Figure 2.1: Map of Wellington showing study locations within the Harbour and on the South Coast ............................................................... 17

Figure 2.2: Line transect at the Quarry ………………...................... 18

Figure 2.3: Quadrat on line trasect at the Quarry .................................. 18

Figure 2.4: Measurements of Patiriella spp. ....................................... 19

Figure 2.5: Mean density of Patiriella spp. per $\mathrm{m}^{2}$....................................... 21

Figure 2.6: Mean density of Patiriella spp. per cobble ............................ 22

Figure 2.7: Proportion of cobbles that were occupied by Patiriella spp. ..... 22

Figure 2.8: Mean density of Patiriella spp., on cobbles that were occupied by at least one Patiriella spp. .......................................................... 23

Figure 2.9: Proportion of occupied cobbles that contain each number of Patiriella spp. ........................................................................ 23

Figure 2.10: Chiton species as a proportion of the total number of chitons surveyed

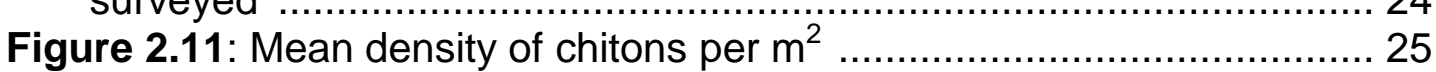

Figure 2.12: Mean density of chitons per cobble..................................... 25

Figure 2.13: Proportion of cobbles that were occupied by chitons ............. 26

Figure 2.14: Mean density of chitons, on cobbles that were occupied by at least one chiton ........................................................................... 26

Figure 2.15: Proportion of occupied cobbles that contain each number of chitons

Figure 2.16: Correlation between the number of Patiriella spp. and chitons per cobble ............................................................................ 27

Figure 2.17: Proportion of Patiriella spp. feeding ................................... 28

Figure 2.18: Proportion of Patiriella spp. on each substrate that were feeding and not feeding ...................................................................... 29

Figure 2.19: Mean size of Patiriella spp. ........................................... 29

Figure 2.20: Distribution of Patiriella spp. sizes ................................... 30

Figure 3.1: Bait station placed below waterline at Moa Point .................... 43

Figure 3.2: Aquaria holding supplemented, non-supplemented and unfed treatments prior to testing for supplemental food attraction ................. 45

Figure 3.3: Mean number of Patiriella spp. arriving at bait stations ............ 48

Figure 3.4: Relationship between the number of Patiriella spp. and the number of whelks on each bait station ............................................. 49

Figure 3.5: Mean weight gain of Patiriella spp. after one week in unfed, nonsupplemented and supplemented treatments ................................... 50

Figure 3.6: Proportion of Patiriella spp., from different week-long feeding regimes, which were feeding after being offered carrion .................... 51

Figure 3.7: Proportion of Patiriella spp., from different week-long feeding regimes, which came into contact with the mussel ............................ 51

Figure 3.8: Mean time that Patiriella spp. stayed in contact with the mussel after first contact for varying week-long feeding regimes 
Figure 3.9: Proportion of Patiriella spp., from the Wellington Harbour and the Wellington South Coast, which were feeding at the end of the experiments

Figure 3.10: Proportion of Patiriella spp., from the Wellington Harbour and the South Coast, which came into contact with the mussel

Figure 3.11: Mean time that Patiriella spp. from Wellington Harbour and Wellington South Coast stayed in contact with the mussel after first contact

Figure 4.1: Experimental setup for growth and mortality response to competition

Figure 4.2: Mean weight change of Patiriella spp. subjected to intra- and interspecific competition during six-week non-supplemented feeding experiments in winter

Figure 4.3: Seasonal comparison of mean weight change of Patiriella spp. during six-week intraspecific feeding experiments where Patiriella spp. were not fed supplemental food

Figure 4.4: Mean weight change of Patiriella spp. during six-week intraspecific feeding trials for supplemented and non-supplemented individuals in spring

Figure 4.5: Mean cumulative weight change of Patiriella spp. during tenweek feeding experiments in spring

Figure 4.6: Mean weight change of Chiton glaucus subjected to intra- and interspecific competition during six-week feeding experiments

Figure 4.7: Mean gonad indices of Patiriella spp. after ten weeks at varying densities with and without supplementation

Figure 4.8: Mean pyloric caeca indices of Patiriella spp. after ten weeks at varying densities with and without supplementation

\section{List of Tables}

Table 3.1: Average number of the most abundant species per bait station in Wellington Harbour and on the Wellington South Coast

Table 4.1: Summary of treatments for competition experiments .................68

Table 4.2: Summary of densities for competition experiments ....................69

Table 4.3: Results for ANOVAs investigating the change in weight of

Patiriella spp. 


\section{1}

\section{Introduction}

\subsection{Intertidal Cobble Habitats}

\section{Intertidal Systems}

The coastal marine ecosystem is an important resource to New Zealand, both economically and ecologically and it is a significant part of New Zealand's local culture and international image. The intertidal patterns and community dynamics on New Zealand's coast are similar to those in other temperate intertidal regions of the world (Menge, 1999).

The marine intertidal environment changes rapidly from low to high on the shore, which creates varying physical gradients and species assemblages (Bertness \& Leonard, 1997). The upper limits of a species' zone are often controlled by physical factors such as desiccation, higher temperature and strong solar radiation, while the lower limits are more often set by biotic factors, such as competition and predation (Connell, 1961; 1972). Therefore the harsher physical conditions higher on the shore tend to decrease the diversity and abundance of species at higher tidal heights (Davidson et al., 2004). This large variation in species assemblages within relatively small areas makes the intertidal ideal for studying general ecological community theories that would otherwise have to be tested on a large spatial scale (Underwood et al., 2000). Species are also relatively easy to manipulate as most are either sessile or slow moving. The intertidal rocky bench habitat has received the most attention from ecologists as it is easily accessed and 
therefore many of its species and interactions are well studied (Connell, 1972; Underwood, 2000).

\section{Cobble Habitats}

By contrast with intertidal rocky benches, intertidal cobble fields are understudied, yet contain their own unique species assemblages and ecological patterns and underlying processes. Early testing within boulder fields led to the intermediate disturbance hypothesis, which states that species diversity is greatest where there is an intermediate level of disturbance (Sousa, 1979). This was discovered by studying the species assemblages on varying sized cobbles that were subjected to different levels of disturbance. However, the intermediate disturbance hypothesis does not appear to hold for sheltered cobble fields. Factors such as aggregation, vulnerability to predators and desiccation appear to be more important in defining species assemblages in less wave exposed areas (McClintock et al., 2007).

The most abundant animals in cobble fields are molluscs and echinoderms (Chapman, 2002b). However, species assemblages and abundances vary between shore level, sites and times (Chapman, 2002b; Cruz-Motta, 2005). Distribution of many species is patchy (Chapman, 2005), but no suitable explanation has been found for this pattern. The most variation in assemblages appears to be among individual cobbles (Chapman, 2002a). Cobble size, substrate under the cobble and presence of algae or other animals appears to have no effect on the diversity of species (Grayson \& Chapman, 2004; Chapman, 2005). The physical composition of cobbles may affect the assemblages that inhabit them (Le Hir \& Hily, 2005; Smoothey \& Chapman, 2007), but surface complexity has been found to have little effect on colonisation (McGuiness \& Underwood, 1986).

The limited study to date on intertidal cobble fields is likely related to the difficulties imposed by this unique habitat. Cobbles and the surface beneath cobbles, provide a three-dimensional area, which creates complexity and 
added complications when studying this environment. Cobbles are discrete patches of habitat (Chapman, 2002b) adding the variation between the top and bottom of cobbles to the variation found between shore heights (CruzMotta, 2005). Many of the mobile species in cobble fields do not have a permanent home (Cruz-Motta, 2005). Diel movement of some species includes sheltering underneath cobbles during the day and moving in search of food during the night. This adds to the difficulties in understanding the patterns and processes involved in shaping intertidal cobble communities.

Intertidal cobble fields are often sparsely scattered along a coastline (Chapman, 2002a), however they are common within Wellington Harbour and on the Wellington South Coast of New Zealand. The accessibility of these habitats makes them vulnerable to anthropogenic disturbance. It is therefore important that we study the patterns and processes within cobble fields to obtain a clearer understanding of the impacts of human activities on coastal marine ecosystems.

\subsection{Biological Processes}

To fully understand anthropogenic impacts and to conserve and manage ecosystems, such as coastal intertidal cobble fields, the patterns and processes that shape communities must be understood (Underwood, 2000). Hairston et al. (1960) theorised that, as there is an abundance of plants, herbivore numbers must be controlled by predators, therefore allowing plants to thrive. This became known as the "world is green" hypothesis and initiated debate about whether communities are regulated from the top-down (predation) or bottom-up (resource limitation). Community regulation is more complex than first suggested by the "world is green" hypothesis and it appears that both top-down and bottom-up controls are important and that their relative importance varies in space and time (Turkington, 2009).

Predation can play an important role in shaping communities and increasing species diversity. For example, removal of the predatory seastar Pisaster ochraceus led to competitive exclusion of other species by the mussel 
Mytilus californianus, in a study on the Pacific Coast of North America (Paine, 1966). A similar study in New Zealand found that the mussel Perna canaliculus outcompeted other species for space when its predator, the seastar Stichaster australis, was removed (Paine, 1971). Paine (1980) suggested that seastars are strong interactors, but that their influence is sporadic and unpredictable. Grazing by herbivores is also important in structuring communities as shown by the removal of grazing urchins, which increased algal abundance and species diversity (Paine \& Vadas, 1969). Predation can reduce the intensity of competition, but in harsher conditions predators may be absent, causing competition to be more important (Connell, 1972).

Underwood (2000) suggested that there were three types of competition on rocky shores: pre-emptive, interference and exploitative. Pre-emptive competition occurs where settlement of one species prevents another from settling. An example of this is where algal fronds sweep rock surfaces and prevent the settlement of barnacles (Dayton, 1971). Interference competition is where one species directly harms or kills another, for example, where the barnacle Balanus balanoides overgrows the barnacle Chthamalus stellatus (Connell, 1961). Exploitative competition occurs when there is insufficient food to support all animals needing it. For example, the seastar Pisaster ochraceus limits the growth of the seastar Letasterias hexactis, because $P$. ochraceus is a more efficient predator (Menge, 1972). Space is the most limiting resource for most sessile organisms in the intertidal (Dayton, 1971) and perhaps food resources are most important for mobile species, such as seastars.

The strength of predation and competition is also affected by larval supply and recruitment. Recruitment is spatially and temporally variable and this can affect community dynamics (Connolly \& Roughgarden, 1999). Limited larval supply can cause predators to become temporarily locally absent, enabling prey to settle and survive to a size at which they are no longer vulnerable to predation, which can result in pulses in prey abundance (Underwood, 2000). Larval populations are determined by adult stock sizes, which are affected by 
interactions such as competition and predation (Connolly \& Roughgarden, 1999). However, larval transport processes are important too. For example, upwelling and downwelling can affect the arrival of larvae (Connolly \& Roughgarden, 1998).

Biological interactions can also be positive, with one species facilitating the growth and survival of another species. Large animals can provide shelter for smaller animals. For example, mussel beds provide suitable habitat for small animals by reducing water movements and trapping detritus (Connell, 1972). Algal cover can also benefit other species by reducing physical stressors, such as high temperature and desiccation, leading to higher recruitment, growth and survival under algae canopies (Bertness et al., 1999). Biological interactions are mediated by physical conditions, with greater physical pressures causing decreased biological interactions (Connell, 1972; Bertness et al., 1999). At high tidal heights physical stress is greater, with higher temperatures and greater desiccation; therefore refuges, such as algal canopies and mussel beds are more important than at low tidal heights where consumer pressure is greater (Bertness et al., 1999). Both biological and physical processes interact to affect community structure. For example, disturbance, disease or predation can lead to reductions in competitors, which can have indirect effects on other species (Underwood, 2000).

\subsection{Omnivores and Scavengers}

\section{Omnivores}

Omnivores interact at multiple trophic levels and therefore have potential to influence the processes and patterns within a community. There has been some debate, mostly theoretical, about how omnivory may affect the stability of food webs (HilleRisLambers et al., 2006). Due to links to more than one trophic level, omnivores were thought to be rare and to destabilize complex food webs (Pimm \& Lawton, 1978). However, omnivory is now shown to be common and to add to web complexity, causing a dampening of fluctuations and an increase in stability (McCann \& Hastings, 1997; Coll \& Guershon, 2002). The complexity of a food web has been suggested as the most 
important aspect driving community and web dynamics (Polis \& Strong, 1996).

Many intertidal animals that are considered herbivores actually consume a high number of animals and may therefore be considered omnivores (Camus et al., 2008). In a study of "herbivorous" Mollusca, Echinodermata and Crustacea, Camus et al. (2008) found that more than $40 \%$ of the items consumed were animal prey. This consumption may be incidental or opportunistic, but still has the potential to affect community dynamics. The seastar Patiriella regularis is often considered herbivorous, but may consume micro-organimsms, as well as opportunistically scavenging on carrion (Crump, 1971) and is therefore considered an omnivore within this study. Previous studies within the intertidal have focused on the predatory role of carnivorous seastars (Gaymer et al., 2002; McClintock et al., 2008), but further study is required on the ecological role of herbivores and omnivores in this group.

\section{Scavengers}

Scavengers feed on dead or decaying animals that they have not killed themselves and they therefore play an important role in the community by recycling nutrients (Selva \& Fortuna, 2007). Britton and Morton (1994) suggest that true scavengers must be able to detect and deliberately move towards carrion and eventually consume either part or all of it. Nearly all phyla of marine and terrestrial animals contain scavenger species (Britton \& Morton, 1994). Some of the more common and well studies terrestrial scavengers include jackals, crows, ravens and vultures (Heinrich, 1988; Deygout et al., 2009; San et al., 2009). Marine scavengers include fish, seabirds, crabs, whelks and seastars (Britton \& Morton, 1994; Moore \& Howarth, 1996; Furness et al., 2007), including those in the genus Patiriella.

Carrion is generally considered an unpredictable and scarce resource (Britton \& Morton, 1994) and, therefore, nearly all scavengers are facultative (Britton \& Morton, 1994; Selva \& Fortuna, 2007). Vultures appear to be the 
only obligate vertebrate scavenger (Deygout et al., 2009). Carrion supply is affected by anthropogenic influences that may increase the amount and frequency of carrion availability (McKillup \& McKillup, 1997). Discarded bycatch and animals killed by beam trawling can dramatically increase the supply of carrion in the deeper marine environment (Britton \& Morton, 1994; Evans et al., 1996). Changes in the availability of carrion can affect scavenger population sizes and negatively impact community dynamics. For example, increases in the burrowing gastropod scavenger, Nassarius dorsatus, may affect the structure of soft sediment communities because of physical disturbance of the substratum (McKillup \& McKillup, 1997). Increases in scavenger numbers may also have an impact on a scavenger's predators and competitors (Evans et al., 1996) and play a role in controlling the dominant species in the community. Therefore, studying the role of scavenger species, such as Patiriella regularis is important in understanding the impacts of changes to carrion supply.

\subsection{Patiriella}

\section{The Patiriella Genus}

Patiriella are a diverse group of asterinid seastars, which are mainly found in Southern Australia and New Zealand. Unlike most other seastar species, which are predatory, Patiriella spp. are generally considered to be herbivorous or opportunistic omnivores. Most species appear to feed only on micro-algae (Branch \& Branch, 1980; Arrontes \& Underwood, 1991; Chen \& Chen, 1993; Jackson et al., 2009), while other species have been recorded opportunistically feeding on carrion (Keough \& Butler, 1979; Stevenson, 1992). Some Patiriella species may also infrequently feed on live prey, such as ascidians, sponges (Keough \& Butler, 1979) and possibly other Patiriella species (Stevenson, 1992).

Patiriella regularis and Patiriella mortenseni are the only endemic New Zealand species (O'Loughlin et al., 2002), while there are nine species in Australia (Byrne \& Anderson, 1994). Size varies considerably between Patiriella species. Patiriella parvivipara is the smallest known seastar with an 
average adult radius of $5 \mathrm{~mm}$ (Byrne, 1996) and $P$. regularis is the largest Patiriella species with an average adult radius of $80 \mathrm{~mm}$ (Byrne \& Barker, 1991; Chen \& Chen, 1992). Reproductive strategies are also very diverse in the Patiriella genus (Palumbi, 1997). The small $P$. parvivipara and $P$. vivipara are simultaneous hermaphrodites, which incubate their young to produce live crawl-away juveniles (Byrne, 1996). By comparison P. calcar, P. brevisina, $P$. gunii, $P$. pseudoexigua and $P$. exigua are free spawners that produce lecithotrophic larvae (Chen \& Chen, 1992; Byrne, 1996; Byrne et al., 2001). The New Zealand species, $P$. regularis and $P$. mortenseni, are the only Patiriella species that produce planktotrophic larvae (Byrne \& Barker, 1991; Byrne, 1996). Lecithotrophic larvae gain nutrition from yolk supplied by the egg and planktotrophic larvae receive their nourishment from actively feeding within the plankton.

\section{Patiriella in New Zealand}

Patiriella regularis is New Zealand's most common rocky shore seastar and is found in a wide range of coastal habitats (O'Loughlin et al., 2002), including low intertidal cobble fields. It is abundant in shallow waters of both the North and South Islands and has been reported to a depth of $100 \mathrm{~m}$ (Crump, 1971). Patiriella regularis also occurs on Stewart Island, the Chatham Islands and in Tasmania, where it was introduced from New Zealand (Waters \& Roy, 2004).

New Zealand Patiriella exhibit considerable variation in colour. Patiriella regularis are predominantly olive-green with yellow, orange, mauve and brown hues and $P$. mortenseni can be dark red-brown to dark grey to blue (O'Loughlin et al., 2002). Both species generally have five rays, but some individuals may have up to seven. Patiriella mortenseni is less tolerant of low salinity and is usually a deeper-dwelling species than $P$. regularis (Barker \& Russell, 2008). There is a single study published on the adult ecology of $P$. regularis, which provides information on its feeding and reproductive ecology (Crump, 1971), but further research on $P$. regularis and $P$. mortenseni appears to be limited. 
To feed, $P$. regularis everts its cardiac stomach through its mouth to cover most of its oral surface. Organic material trapped beneath its stomach is digested with powerful digestive enzymes (Crump, 1971). These seastars are opportunistic omnivores and appear to feed on a variety of organisms. They are most often observed feeding on crustose coralline algae, but may also consume microscopic food items, such as diatoms and newly settled invertebrates (Crump, 1971; Naylor \& McShane, 2001). Patiriella regularis are also scavengers and gain much of their energy from occasional carrion food (Crump, 1971).

Previously considered as one species, Patiriella within New Zealand have recently been recognised as two separate species, $P$. regularis and $P$. mortenseni (O'Loughlin et al., 2002). Even more recently, phylogenetic analysis has separated these species further into different genera, with $P$. mortenseni being moved to the genus Meridiastra and $P$. regularis remaining within the Patiriella genus (O'Loughlin \& Waters, 2004). However, for the purposes of this thesis, these two species will continue to be considered within the Patiriella genus, as this still appears to be the common usage. Patiriella regularis and $P$. mortenseni are not easily distinguishable from each other (Kate Neill, pers. comm., 2009) and therefore both New Zealand species of Patiriella will be referred to collectively as Patiriella spp. throughout this thesis.

\subsection{Wellington Harbour and South Coast}

Wellington is at the southern end of New Zealand's North Island $\left(41^{\circ} 16^{\prime} \mathrm{S}\right.$, $174^{\circ} 51^{\prime} \mathrm{E}$ ) and contains a relatively large sheltered harbour. Its coastline is exposed to the Cook Strait, which separates the North and South Islands of New Zealand. The intertidal areas of Wellington Harbour and the Wellington South Coast include many rocky bench and cobble field habitats, which are inhabited by Patiriella spp. 
Wellington Harbour has a surface area of $85 \mathrm{~km}^{2}$ and is relatively land-locked, with the southern end open to the Cook Strait through a shallow channel with a width of $1.8 \mathrm{~km}$ (Maxwell, 1956; Heath, 1977). Maxwell (1956) suggests that the Harbour channel is large enough to ensure good mixing of Harbour water with that from outside the Harbour. However, Booth (1975) found that the Wellington Harbour is clearly distinct from the Cook Strait, providing different hydrological conditions within the Harbour compared to outside it. Tides are semi-diurnal and flood the Harbour in a clockwise direction and ebb anticlockwise (Booth, 1975). Other inputs include freshwater from the Hutt River and Ngauranga and Korokoro streams, which can affect surface salinities and turbidity (Booth, 1975). However, the impact of these freshwater inputs appears to be significant only during flooding (Maxwell, 1956). Water in the Wellington Harbour appears to be well mixed (Booth, 1975).

Outside the Harbour is Wellington's South Coast, which is an exposed, predominantly rocky, shoreline open to the Cook Strait. Water from three sources mix to fill the Cook Strait. The subantarctic Southland current contains cool, low salinity water from the eastern coast of the South Island (Heath, 1971). This Southland Current mixes with the warmer, subtropical waters of the East Cape Current, from the east coast of the North Island, and the D'Urville Current, originating from the west coast of the South Island (Heath, 1971). Depending on meteorological conditions, tidal currents within the Cook Strait can be very strong (Bowman et al., 1983; Heath, 1985) and these currents flow along Wellington's South Coast (Gardner, 2000).

Biotic differences have also been observed between Wellington Harbour and the Wellington South Coast. Primary productivity is low along the Wellington South Coast, with lower quality and quantity of particulate organic matter than within Wellington Harbour (Gardner, 2000; Helson et al., 2007). Species abundance also varies between regions (Maxwell, 1956), with the most obvious example being the near absence of mussels on the Wellington South Coast. Within Wellington Harbour the rocky intertidal zone is generally dominated by three species of mussel: the ribbed mussel Aulacomya 
maoriana, the blue mussel Mytilus galloprovincialis and the greenshell mussel Perna canaliculus (Helson \& Gardner, 2004). There are also large differences in the distribution of barnacles between the regions. The overall abundance is sparse and patchy on the South Coast and dominated by Chamaesipho brunnea, whereas barnacles are dense in Wellington Harbour and dominated by a different species, C. columna (Demello, 2007). Intertidal community differences may be due to variation in larval supply or recruitment dynamics between the regions (Phillips \& Hutchison, 2008) or high mortality on the South Coast due to low planktonic food supply (Helson et al., 2007), or a combination of factors. Due to these large differences in community structure over a relatively small spatial scale, the Wellington Harbour/South Coast has been a model system for several ecological studies (e.g. (Gardner, 2000; Helson \& Gardner, 2004; Helson et al., 2007; Phillips \& Hutchison, 2008; Shima \& Swearer, 2009a; b); however no studies to date have examined communities in intertidal cobble fields between the two locations.

\subsection{Aims and Objectives}

The aim of this study is to better understand the ecological role of Patiriella spp. within intertidal cobble fields in the Wellington region. Interactions and community dynamics within intertidal cobble fields, as with any ecosystem, are complex and require understanding of many of its inhabitants. This study attempts to provide further knowledge of the interactions and roles of Patiriella spp., which is one of the common and abundant inhabitants of intertidal cobble fields. This aim was achieved by:

1) Observing the density, size distributions and feeding habits of Patiriella spp. in intertidal cobble fields within Wellington Harbour and on the Wellington South Coast.

2) Examining the scavenging behaviour of Patiriella spp. and comparing this behaviour between individuals from Wellington Harbour and Wellington South Coast.

3) Conducting experiments to test the effect of intraspecific and interspecific competition for food on the mortality, growth and 
reproductive potential of Patiriella spp and its potential competitor, Chiton glaucus. 


\section{2}

\section{Density, Feeding Habits and Size of Patiriella spp. within Intertidal Cobble Fields}

\section{Abstract}

Intertidal cobble fields contain a diversity and abundance of both sessile and mobile species, yet these systems remain understudied around the world and especially in New Zealand. The New Zealand cushion stars Patiriella regularis and Patiriella mortenseni are commonly found within intertidal cobble fields. These Patiriella species are opportunistic omnivores that appear to feed on crustose coralline algae and micro-organisms, while supplementing their diet by scavenging on carrion. Surveys were conducted within intertidal cobble fields at three sites within Wellington Harbour and on the Wellington South Coast, which were then repeated after three months. These surveys examined the density, size and feeding substrate of Patiriella spp. and the density and species of chitons, as their potential competitors. There was little difference in density of Patiriella spp. or chitons in Wellington Harbour compared with the Wellington South Coast. Distribution of both Patiriella spp. and chitons was patchy and a negative correlation was found between Patiriella spp. and chiton density per cobble, suggesting competition between these taxa. A greater proportion of Patiriella spp. were feeding on the South Coast compared with the Harbour and of those feeding, crustose coralline algae appeared to be the preferred substrate. Sizes of Patiriella spp. were marginally different between regions, with smaller seastars within the Harbour compared with the South Coast. The cause of feeding and size 
differences between the regions remains unclear, but food availability is likely to play an important role. Patiriella spp. have the potential to influence community dynamics by decreasing crustose coralline algal cover, while their distribution may be affected by the presence of carrion.

\subsection{Introduction}

Intertidal cobble fields are common habitats for a variety of sessile and mobile species in temperate low-shore intertidal systems. The macrofauna of cobble fields is diverse and includes crustaceans, molluscs, polychaetes and echinoderms (Chapman, 2005; Cruz-Motta, 2005), of which molluscs and echinoderms are often the dominant groups (Chapman, 2002b). Despite the abundance and diversity of fauna associated with cobble field habitats, cobble fields remain understudied, especially in New Zealand. Observational studies that provide baseline information on the density, feeding habits and size of dominant taxa within intertidal cobble fields are an important first step in increasing our understanding of the processes operating in cobble fields and providing a context for studying mechanisms and processes driving community dynamics in this habitat.

A cobble is a rock with dimensions between 64 and 256mm (McClintock et al., 2007) and anything larger is classified as a boulder (Wentworth, 1922). Within cobble fields, assemblages of organisms are variable at multiple spatial scales. The greatest variation is often among individual cobbles, but there is also variation in assemblages among sites and shore levels (Chapman, 2002b; Cruz-Motta, 2005). Many species move to or away from cobbles as the tidal height changes, with few species having a permanent habitat below cobbles (Cruz-Motta, 2005). Beneath cobbles many species tend to be over-dispersed and patchy, with multiple individuals on a single cobble and many cobbles with no individuals (Chapman, 2002b; 2005). Although multiple studies have attempted to determine why the distribution of mobile animals in cobble fields is patchy, none have yet provided a satisfactory explanation (McGuiness \& Underwood, 1986; Chapman, 2002a; Grayson \& Chapman, 2004; Chapman, 2005). 
Within New Zealand's low intertidal cobble fields common species are the cushion stars Patiriella regularis and Patiriella mortenseni (referred to collectively within this study as Patiriella spp). Currently, very little is known about the patterns of distribution or ecological role of this taxon in the field (Crump, 1971). Patiriella spp. occur around both the North and South Islands of New Zealand, as well as Stewart Island and the Chatham Islands and have been introduced to Tasmania (Waters \& Roy, 2004).

The majority of other Patiriella species are confined to Southern Australia (Byrne \& Anderson, 1994), with one sole species found in the Indo-Pacific Ocean (Chen \& Chen, 1992). Patiriella species vary considerably in size, number of arms and feeding strategies (O'Loughlin \& Waters, 2004), but the most studied difference among species is their reproductive and developmental strategies (Palumbi, 1997). Some species are hermaphrodites with large eggs that develop into crawl-away seastars with no larval stage, while others brood their embryos in the gonad or free spawn producing lecithotrophic larvae (Chen \& Chen, 1992; Byrne, 1996; Byrne et al., 2001). By contrast, $P$. regularis and $P$. mortenseni produce planktotrophic larvae that feed for up to nine weeks before settlement (Byrne \& Barker, 1991; Byrne, 1996).

The only published ecological study examining $P$. regularis in New Zealand found that they are opportunistic omnivores that feed on both microscopic and macroscopic food items (Crump, 1971). Macroscopic food appears to consist primarily of algae and carrion (Crump, 1971), although P. regularis have also been observed feeding on live mussels in field studies (Witman \& Grange, 1998). Microscopic food may consist of diatom films or newly settled invertebrates (Naylor \& McShane, 2001). Crump (1971) found that the size of $P$. regularis was negatively correlated with abundance at sites in Otago. Smaller individuals occurred at one of three sites, which also contained the highest density of animals. Food availability appears to play a role in reproductive potential (Crump, 1971). 
In the Wellington region of New Zealand there are dramatic differences in benthic community structure of intertidal rocky shores between Wellington Harbour and the South Coast (which faces the Cook Strait (Morton \& Miller, 1968)). Most notably, these differences include striking variation in the abundance of sessile invertebrates, with the abundance of mussels and barnacles much reduced on the South Coast relative to Wellington Harbour (Helson \& Gardner, 2004; Phillips \& Hutchison, 2008). Differences in physical conditions between Wellington Harbour and South Coast may also result in differences in the distribution of other organisms, including those inhabiting cobble fields, such as Patiriella spp. However, currently little is known about variation in the abundance of mobile species inhabiting cobble fields across this region.

This chapter examines variation in the abundance and behaviour of mobile species in cobble fields between Wellington Harbour and Wellington's South Coast. Quantitative observations provide an important context for studying mechanisms and processes (Underwood et al., 2000); therefore the aim of this chapter is two-fold: 1) to provide baseline information on the density, feeding habits and size of Patiriella spp. and any other dominant taxa within intertidal cobble fields; and 2) to compare the abundance of Patiriella spp. in cobble fields between Wellington Harbour and Wellington's South Coast. I hypothesised that there would be differences in size, density and feeding between Wellington Harbour and Wellington South Coast populations. Density of Wellington Harbour populations was expected to be larger, due to the greater availability of organic matter (Helson et al., 2007) and therefore more food resources. I expected that a greater proportion of seastars would be feeding within the Harbour, due to the abundance of food. Patiriella spp. were expected to be smaller in the Harbour due to the higher densities (Crump, 1971). Due to observed abundances of chitons, I also hypothesised that there would be a negative correlation between the distribution of Patiriella spp. and chitons, caused by possible competition for algae and microscopic food items. 


\subsection{Methods}

\section{Field Surveys}

Surveys of Patiriella spp. and associated organisms were carried out at three sites in Wellington Harbour (Point Howard, Shelly Bay and Point Halswell) and three sites on the South Coast (Moa Point, Island Bay and the Quarry; Figure 2.1). At each site rocky outcrops were interspersed with patches of cobble. Surveys were conducted within the cobble areas only.

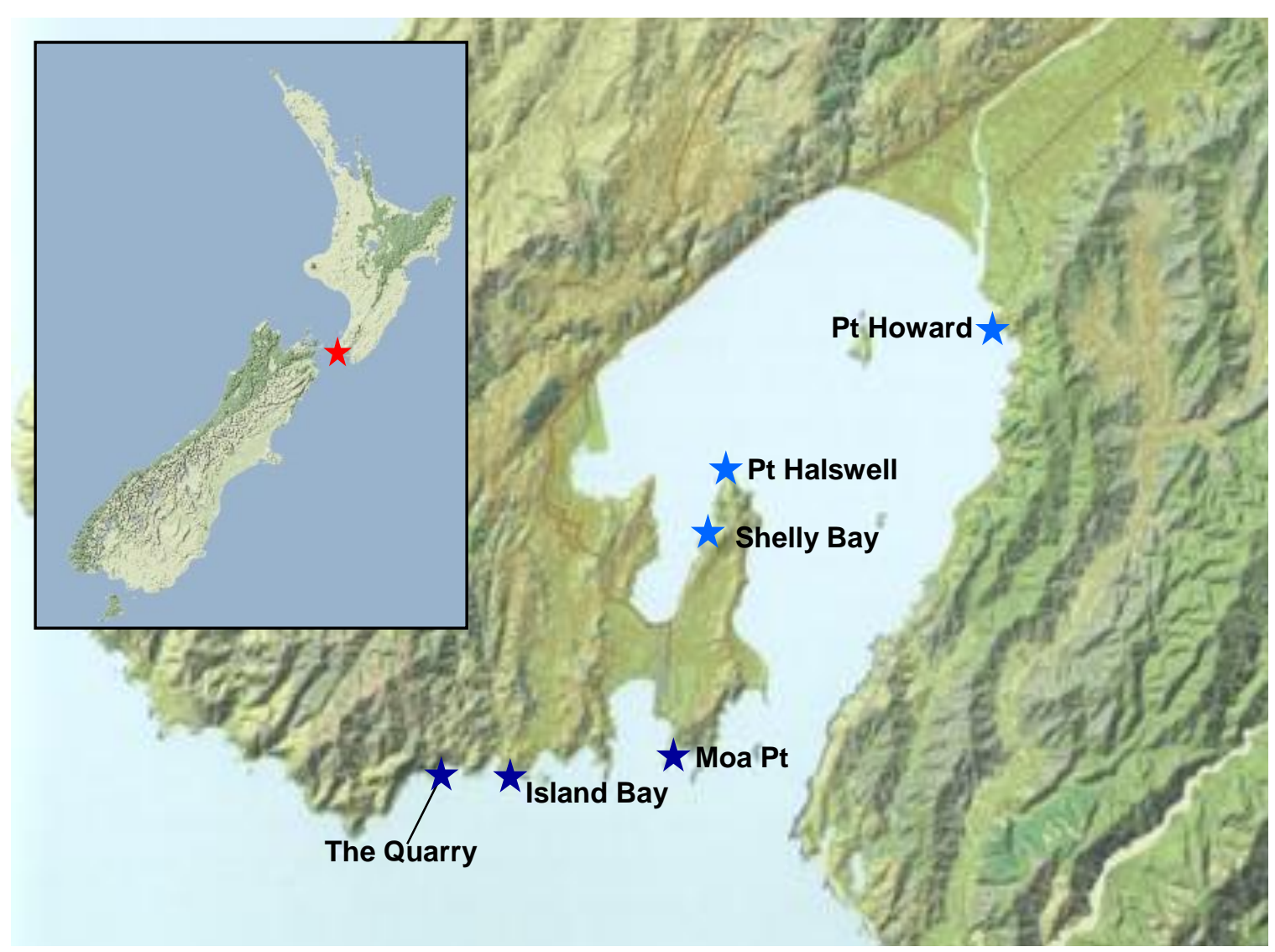

Figure 2.1: Map of Wellington showing study locations within the Harbour (light stars) and on the South Coast (dark stars).

Surveys were conducted within one hour either side of low tide at all six field sites, once in August/September and once in November/December of 2008. Three transect lines $(14-20 \mathrm{~m}$ long) were placed along the waterline (Figure 2.2). Each transect line was separated by at least $5 \mathrm{~m}$. Five $50 \mathrm{~cm} \times 50 \mathrm{~cm}$ quadrats were placed randomly along each transect line (Figure 2.3). Therefore, a total of 15 quadrats, each with an area of $0.25 \mathrm{~m}^{2}$, were 
surveyed at each site. Every cobble within each quadrat, that had a diameter between approximately $60-120 \mathrm{~mm}$, was examined and details of organisms on each cobble were recorded. Organisms generally only inhabited the undersurface of cobbles and so cobbles were overturned to accurately record all organisms.

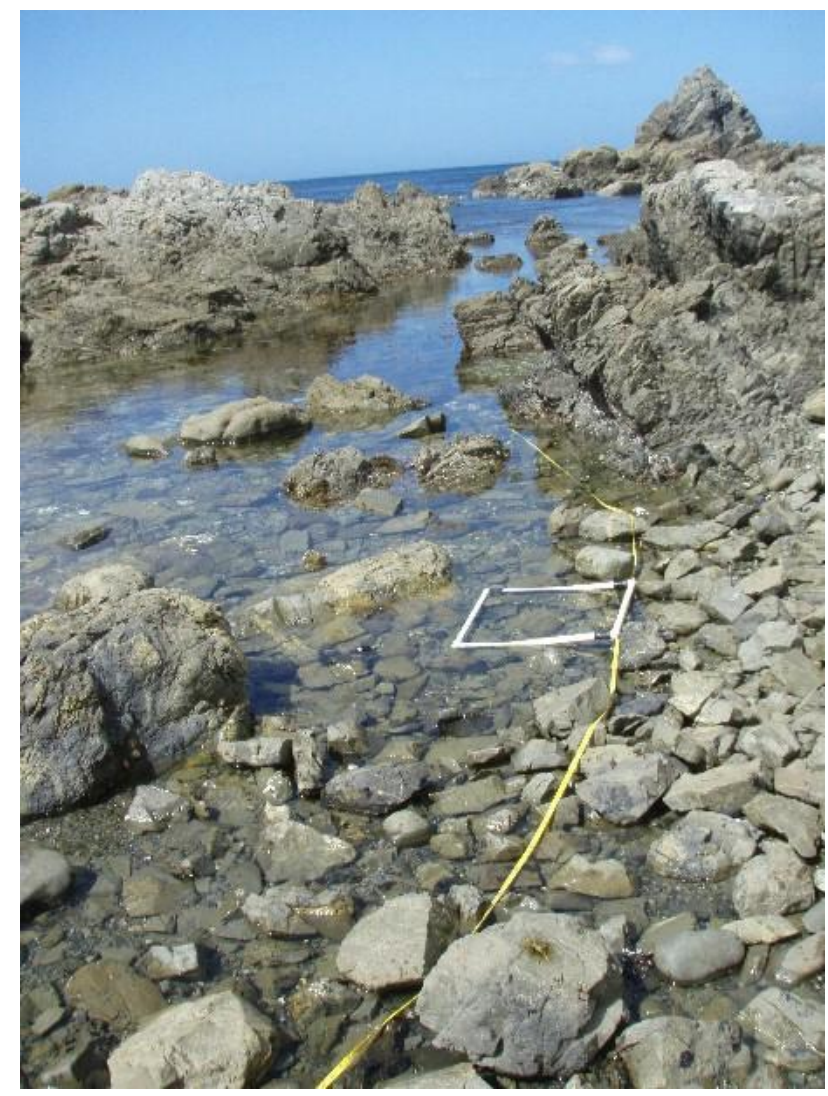

Figure 2.2: Three 14-20 metre line transects were placed along the waterline to survey each site. Line transect shown here at the Quarry on the Wellington South Coast, with a randomly placed quadrat.

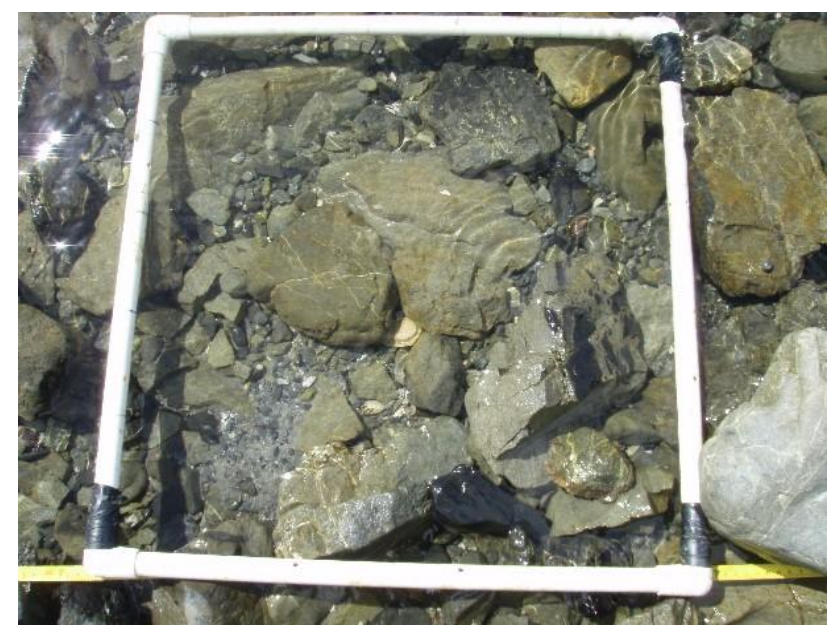

Figure 2.3: Five $0.25 \mathrm{~m}^{2}$ quadrats were randomly placed on each line transect. The quadrat shown here was placed on a line transect at the Quarry site on the Wellington South Coast. 
All macro-organisms were counted and identified to species level where possible and additionally the size and feeding behaviour of Patiriella spp. were recorded. Patiriella spp. size was measured from the tip of the longest arm to the opposite armpit (Figure 2.4). This method was preferred over measuring the radius (Crump, 1971; O'Loughlin et al., 2002) as the armpit was more easily identified than the exact centre of the cushion star. Also recorded were the substrate directly beneath each individual and whether or not the seastar was feeding. A Patiriella spp. was classified as feeding if its second stomach was extruded (Crump, 1971).

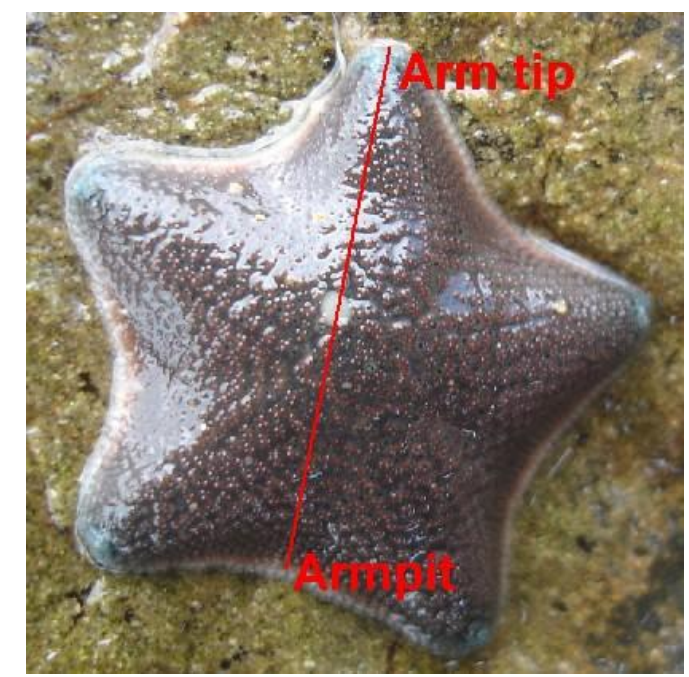

Figure 2.4: Measurements of Patiriella spp. were taken from the tip of the longest arm to the opposite armpit.

\section{Statistical Analysis}

All animals smaller than $5 \mathrm{~mm}$ were excluded from the analysis as they were considered to be too small to have a competitive interaction with Patiriella spp. Data were analysed using JMP 4.0.4.

Six mixed model ANOVAs were used to test: 1) differences in the density of Patiriella spp. per area between the Harbour and South Coast; 2) differences in the density of Patiriella spp. per cobble between the Harbour and South Coast; 3) differences in the density of Patiriella spp. per occupied cobble between the Harbour and South Coast; 4) differences in the density of chitons per area between the Harbour and South Coast; 5) differences in the 
density of chitons per cobble between the Harbour and South Coast; and 6) differences in the density of chitons per occupied cobble between the Harbour and South Coast. Mixed model ANOVAs were run with the following full model: Region (fixed factor, 2 levels), month (random factor, 2 levels), site nested within region (random factor, 3 levels per region) and the month by region interaction. Random factors that were not significant were removed from analysis and the simplest model was used for final interpretations in all cases. ANOVAs were run using the EMS (expected mean squares), but these results were compared to REML (residual maximum likelihood estimation) procedure and in all cases results were qualitatively the same. All figures show untransformed data for ease of interpretation.

Before ANOVAs were run, Levene's test was used to test the assumption of homogeneity of variance and Shapiro-Wilk's was used to test for normality. Density of Patiriella spp. per $\mathrm{m}^{2}$ was log transformed to correct for unequal variances. Data were not normally distributed either before or after transformation, but analysis continued as ANOVA is robust to deviations from normality. Density of Patiriella spp. per cobble, as well as per occupied cobble was non-normal with unequal variances. Log transforming the data did not improve normality or equality of variances; therefore the original untransformed data was used. Size data were log transformed to correct for unequal variances and again data was non-normal. Untransformed data was used to compare the density of cobbles per region as variances were close to equal for this data. Non-parametric tests were not used, as they are not available for mixed models.

Chi-square analysis was used to compare: 1) the number of occupied and unoccupied cobbles between the Wellington Harbour and Wellington South Coast; 2) the numbers of Patiriella spp. feeding between the Wellington Harbour and Wellington South Coast and 3) the numbers of Patiriella spp. feeding on different substrates.

Kendall's tau_b nonparametric correlation was used to test for a correlation between Patiriella spp. and chiton densities per cobble. 


\subsection{Results}

\section{Patiriella spp. Density}

There was no significant difference between the mean densities of Patiriella spp. per unit area in Wellington Harbour compared with the densities on the Wellington South Coast $\left(F_{1,172}=0.06, p=0.84\right.$; Figure 2.5).

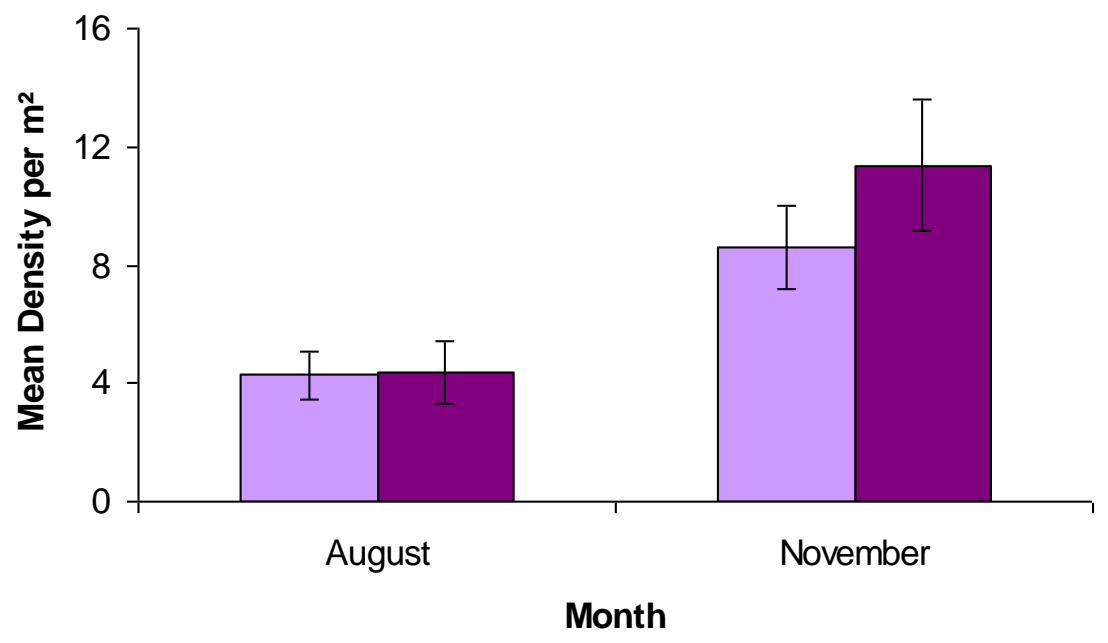

Figure 2.5: Mean density of Patiriella spp. ( $\pm \mathrm{SE}$ ), in the Harbour (light bars) and on the South Coast (dark bars).

The average number of Patiriella spp. on each cobble also did not differ significantly between Wellington Harbour and Wellington South Coast $\left(F_{1,1721}=1.55, \quad p=0.38\right)$. However, the month by region interaction $\left(F_{1,1721}=30.05, \quad p<0.001\right)$ was significant. Post-hoc Tukey tests on the interaction showed that the density per cobble was significantly higher in the Harbour in August, compared to the South Coast in August or either site in November $(p<0.05$; Figure 2.6).

A greater proportion of cobbles were occupied in the Harbour than on the South Coast in August $\left(\chi^{2}=44.10, n=812, p<0.001\right)$, with a similar, but nonsignificant trend, in November $\left(\chi^{2}=3.00, n=917, p=0.08\right.$; Figure 2.7). 


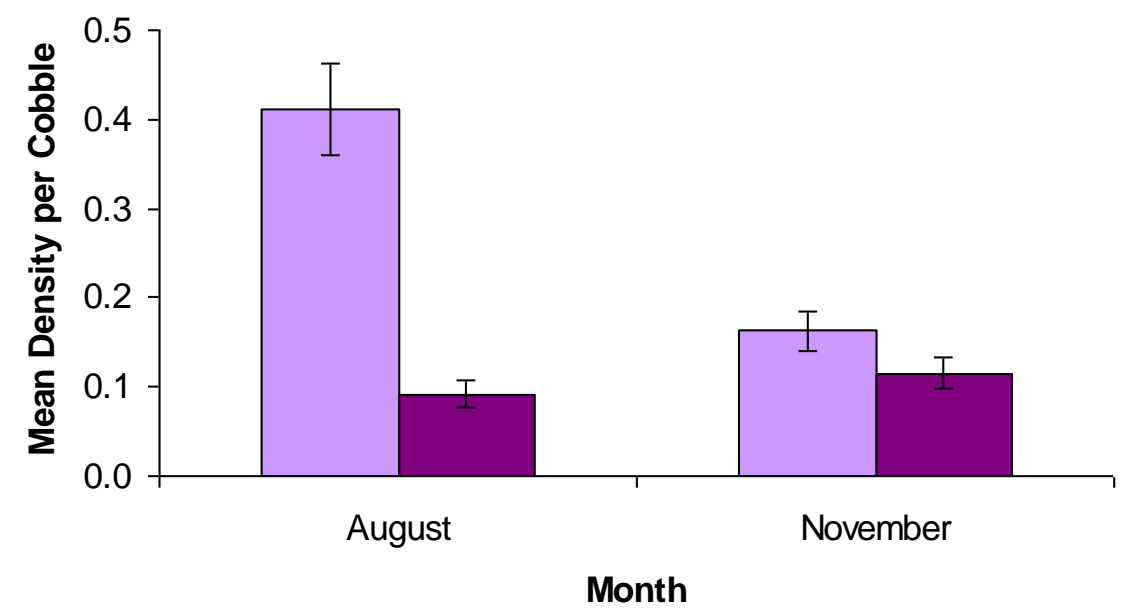

Figure 2.6: Mean density of Patiriella spp. per cobble ( \pm SE) in the Harbour (light bars) and on the South Coast (dark bars).

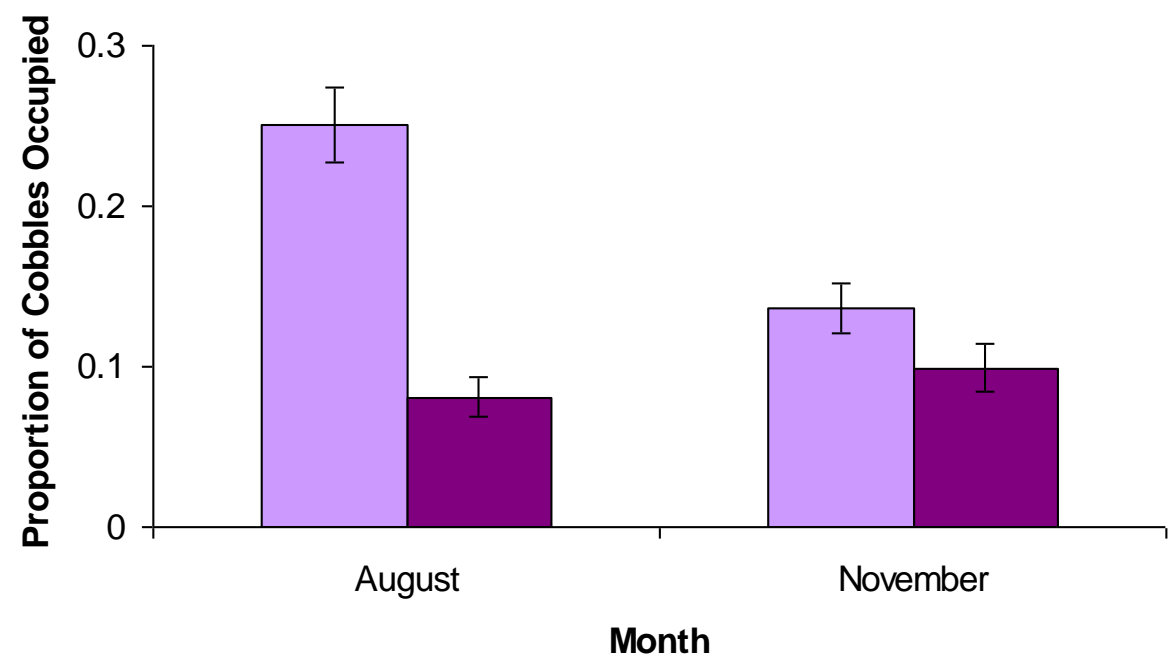

Figure 2.7: Proportion of cobbles that were occupied by Patiriella spp. ( $\pm \mathrm{SE})$ in the Harbour (light bars) and on the South Coast (dark bars).

The average number of Patiriella spp. on occupied cobbles was not significantly different between Wellington Harbour and the Wellington South Coast $\left(F_{1,225}=0.82, p=0.51\right)$. Month by region interaction was significant $\left(F_{1,225}=3.79, p=0.05\right)$ and post-hoc Tukey tests showed that Patiriella spp. density per occupied cobble was significantly higher in the Harbour in August $(\mathrm{p}<0.05$; Figure 2.8).

The majority of occupied cobbles contained only one Patiriella spp., both within the Harbour $(77 \%, S E=3)$ and on the South Coast $(89 \%, S E=3)$, with a 
diminishing frequency of cobbles occupied by increased densities (Figure 2.9). The maximum number of Patiriella spp. per cobble was three at South Coast sites and eight within the Harbour.

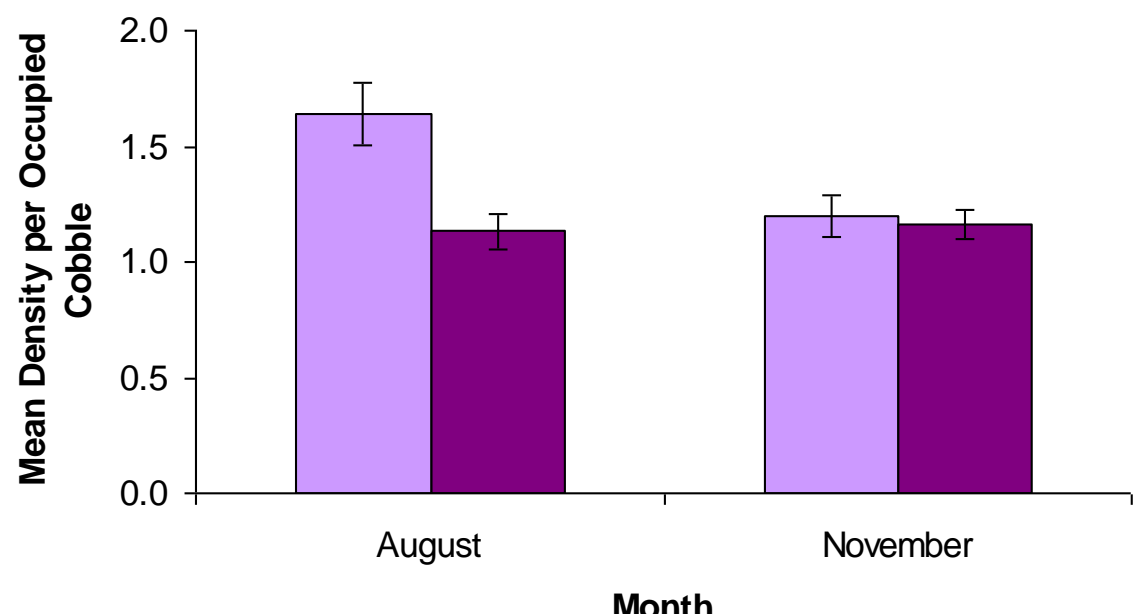

Figure 2.8: Mean density of Patiriella spp. ( $\pm \mathrm{SE}$ ), on cobbles that were occupied by at least one Patiriella spp., in the Harbour (light bars) and on the South Coast (dark bars).

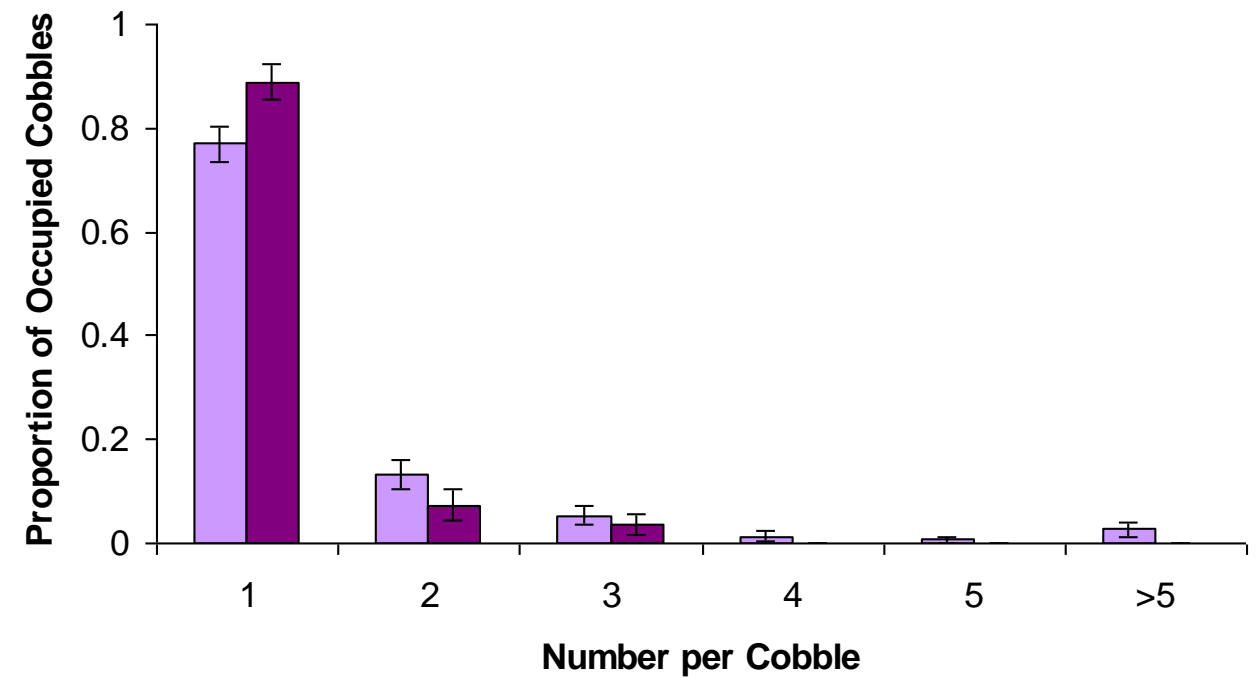

Figure 2.9: Proportion of occupied cobbles that contain each number of Patiriella spp. $( \pm S E)$ in the Harbour (light bars) and on the South Coast (dark bars).

\section{Chiton Density}

Chitons were the most abundant macro-organism within intertidal cobble fields in Wellington Harbour and on the Wellington South Coast and snails and limpets were also relatively common. However, further analysis concentrated only on chitons as these were considered to be the most likely 
competitors for Patiriella spp. due to their algal diets (Creese, 1988), large abundance and similar sizes to Patiriella spp.

In Wellington Harbour the most abundant chiton species was Chiton glaucus (69\%), followed by Sypharochiton pelliserpentis (24\%). These two chiton species were also the most abundant on Wellington South Coast, but there were more S. pelliserpentis (58\%) than C. glaucus (26\%). The numbers of all other species was minimal (Figure 2.10).

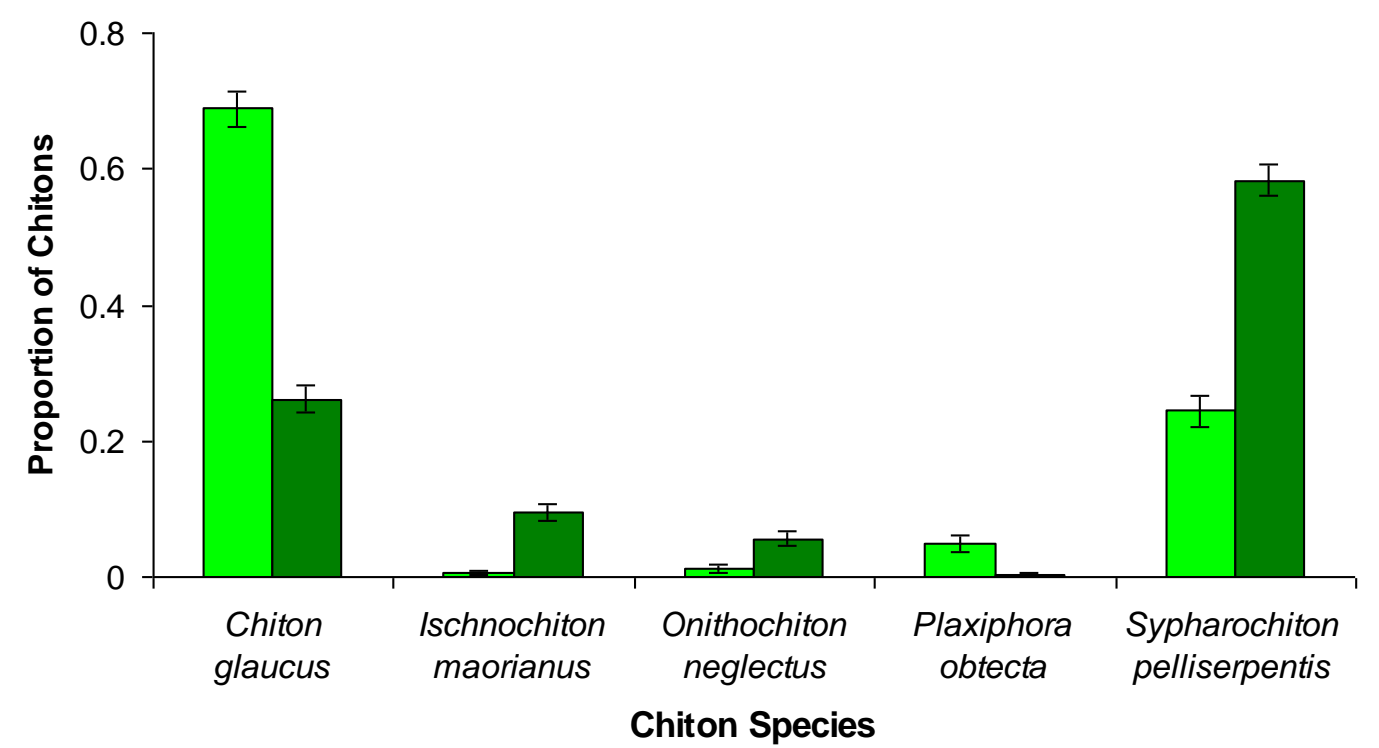

Figure 2.10: Chiton species as a proportion of the total number of chitons surveyed $( \pm S E)$ in the Harbour (light bars) and on the South Coast (dark bars).

There was no significant difference between chiton density per $\mathrm{m}^{2}$ within Wellington Harbour and South Coast $\left(\mathrm{F}_{1,172}=0.01, \mathrm{p}=0.92\right)$. The month by region interaction $\left(F_{1,172}=8.03, p=0.01\right)$ was significant and a post-hoc Tukey test showed that, on the South Coast, there was a significantly higher density of chitons in August compared with November ( $p<0.05$; Figure 2.11).

There was also no difference in density of chitons per cobble between Wellington Harbour and Wellington South Coast $\left(F_{1,1721}=0.001, p=0.98\right)$. The month by region interaction $\left(F_{1,1721}=14.34, p<0.001\right)$ was significant and posthoc Tukey tests showed that this was due to lower chiton densities in the Harbour during November $(p<0.05$; Figure 2.12). 


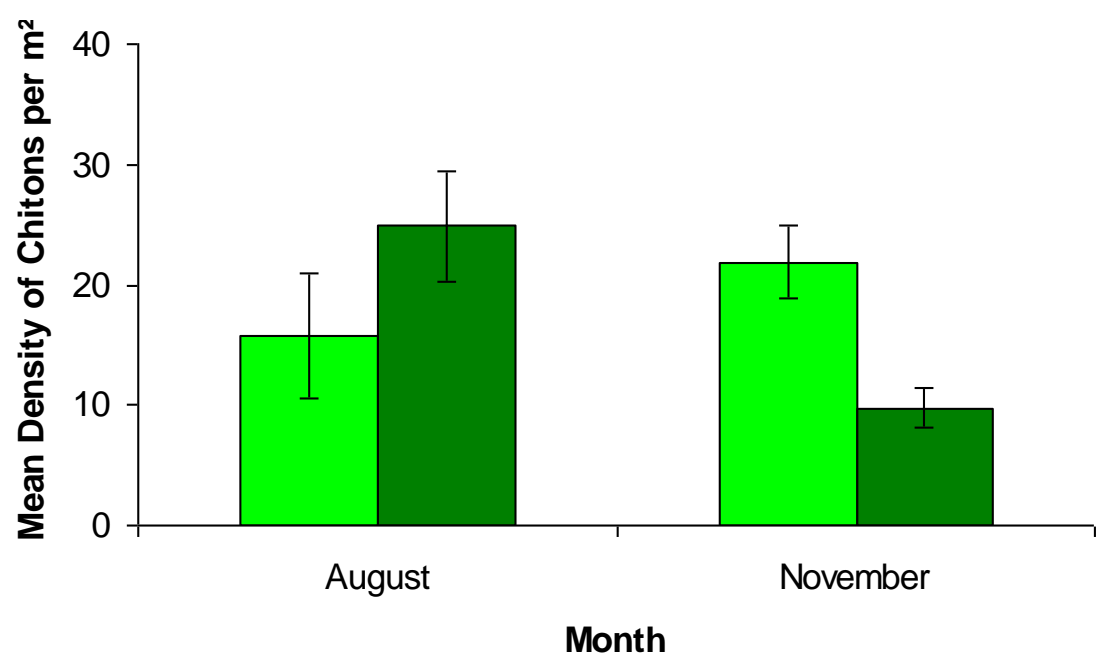

Figure 2.11: Mean density of chitons ( \pm SE), in the Harbour (light bars) and on the South Coast (dark bars).

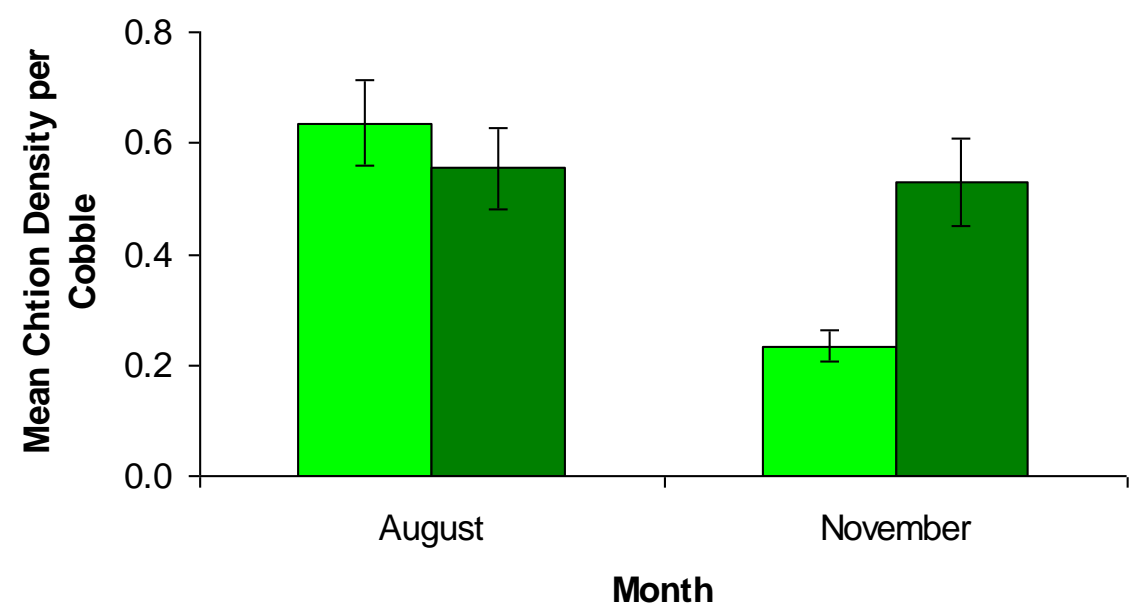

Figure 2.12: Mean density of chitons per cobble ( \pm SE) in the Harbour (light bars) and on the South Coast (dark bars).

Although a greater proportion of cobbles were occupied by chitons in the Harbour than on the South Coast in August $\left(\chi^{2}=16.94, n=812, p<0.001\right)$, no difference in occupied cobbles was found between regions in November $\left(\chi^{2}=1.95, n=917, p=0.16\right.$; Figure 2.13).

The density of chitons on occupied cobbles did not significantly differ between Wellington Harbour and Wellington South $\left(F_{1,371}=2.46, p=0.20\right.$; Figure 2.14). 


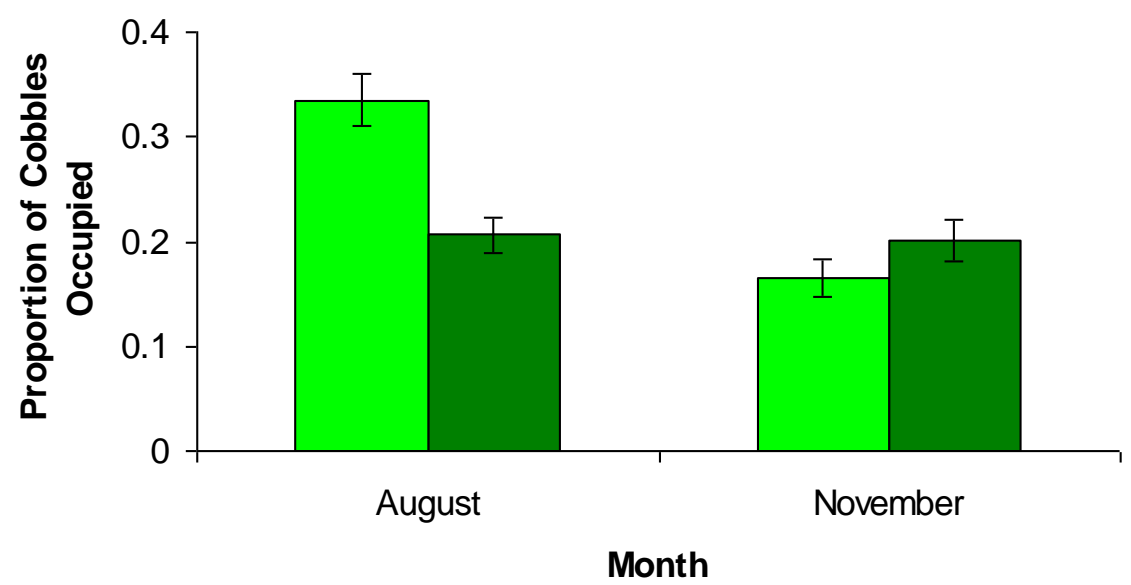

Figure 2.13: Proportion of cobbles that were occupied by chitons $( \pm S E)$ in the Harbour (light bars) and on the South Coast (dark bars).

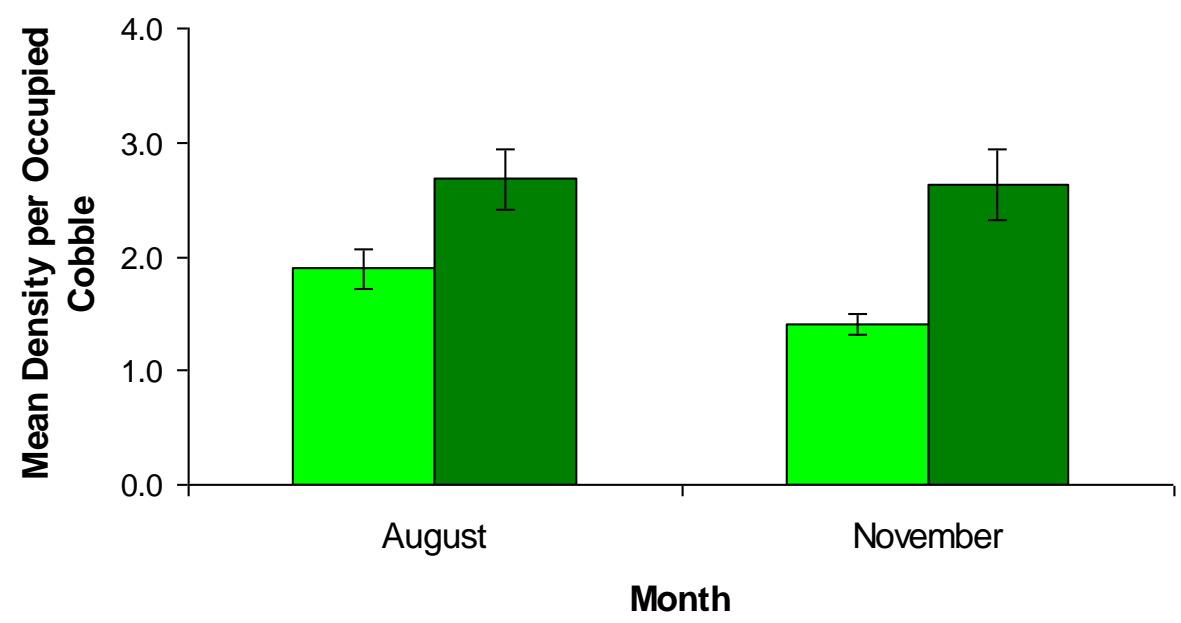

Figure 2.14: Mean density of chitons ( $\pm \mathrm{SE}$ ), on cobbles that were occupied by at least one chiton, in the Harbour (light bars) and on the South Coast (dark bars).

The majority of occupied cobbles contained only one chiton both within Wellington Harbour (63\%) and on Wellington South Coast $(53 \%)$, with a diminishing frequency of cobbles occupied by increased densities (Figure 2.15). 


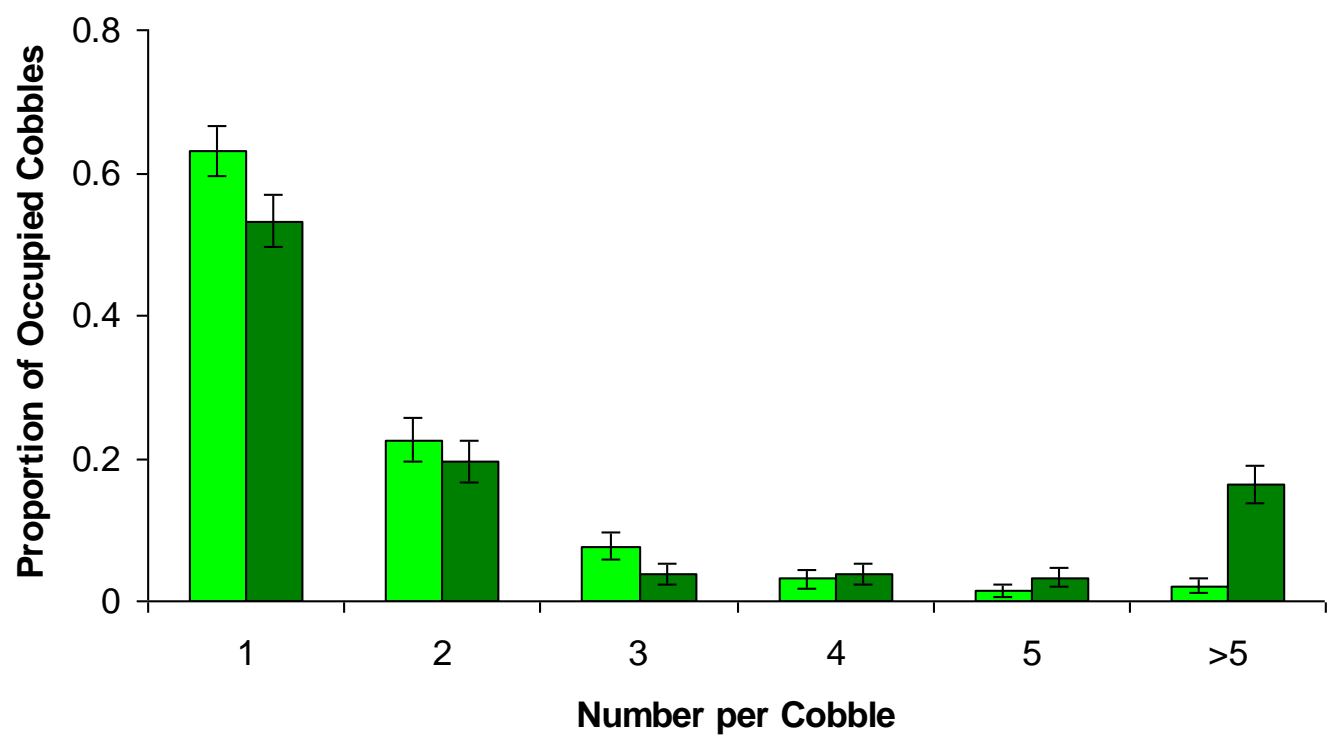

Figure 2.15: Proportion of occupied cobbles that contain each number of chitons $( \pm S E)$ in the Harbour (light bars) and on the South Coast (dark bars).

\section{Associated Organisms}

There was a negative correlation between Patiriella spp. and chiton density per cobble (tau_b=-0.51, $p<0.001$ ). Those cobbles that contained a large number of chitons had no Patiriella spp. and those cobbles with many Patiriella spp. had little or no chitons (Figure 2.16).

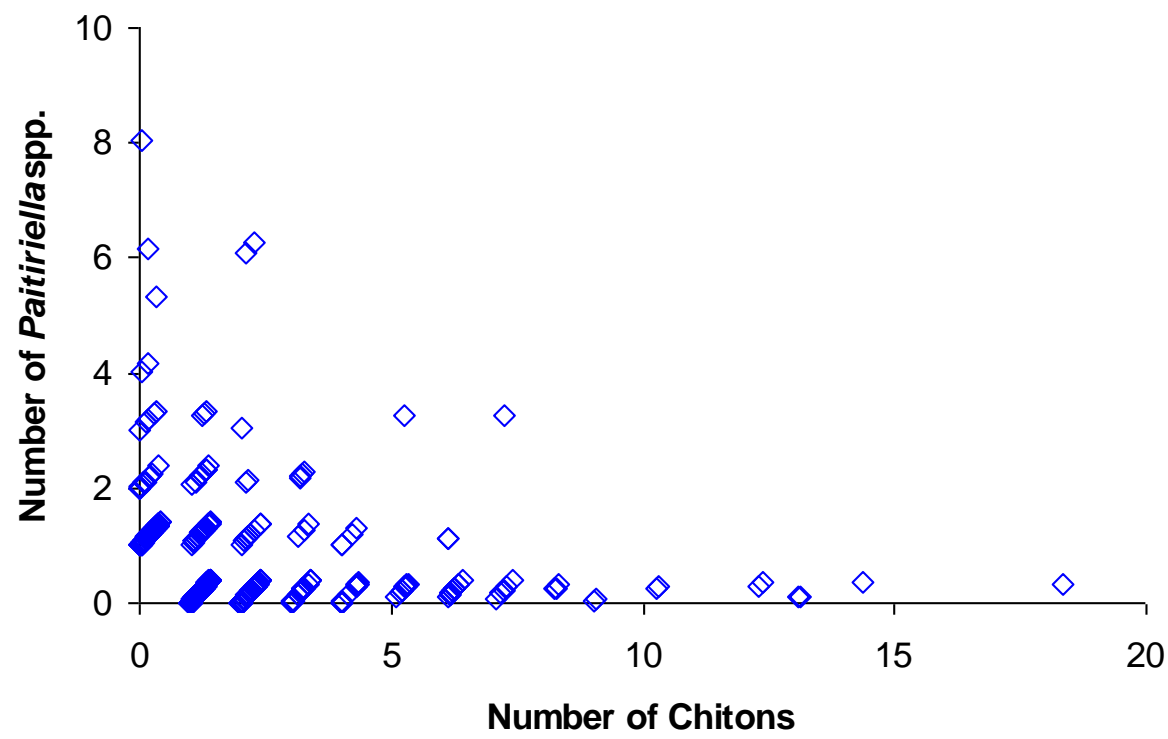

Figure 2.16: Correlation between the number of Patiriella spp. and chitons per cobble (points randomly staggered between -0.2 and 0.2 around their values so that all values at a given point are visible). 


\section{Patiriella spp. Feeding}

The proportion of Patiriella spp. that were feeding varied significantly between the Wellington Harbour and the Wellington South Coast $\left(\chi^{2}=20.94\right.$, $\mathrm{n}=331, \mathrm{p}<0.001$ ), with twice as many Patiriella spp. feeding on the South Coast compared with the Harbour (Figure 2.17).

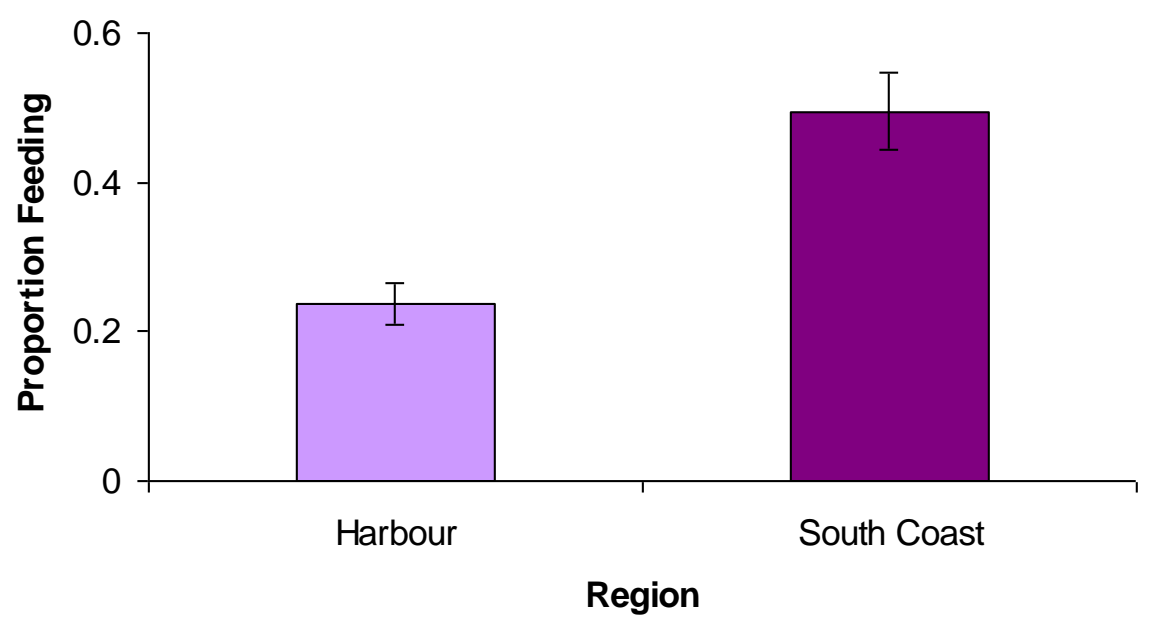

Figure 2.17: Proportion of Patiriella spp. feeding ( \pm SE) in the Harbour and on the South Coast.

The substrate upon which a Patiriella spp. was attached had a highly significant effect on whether or not it was feeding $\left(\chi^{2}=68.79, n=271\right.$, $p<0.001)$. A significantly lower proportion of individuals were feeding $(15 \%)$ compared with those not feeding (85\%) on bare rock and significantly more of the individuals on other substrates were feeding $(69 \%)$ as opposed to not feeding. Other substrates included non-crustose coralline algal groups, tubeworms and bryozoans. Of those Patiriella spp. found on crustose coralline algae, $59 \%$ were feeding, compared with those not feeding on this substrate (Figure 2.18). 


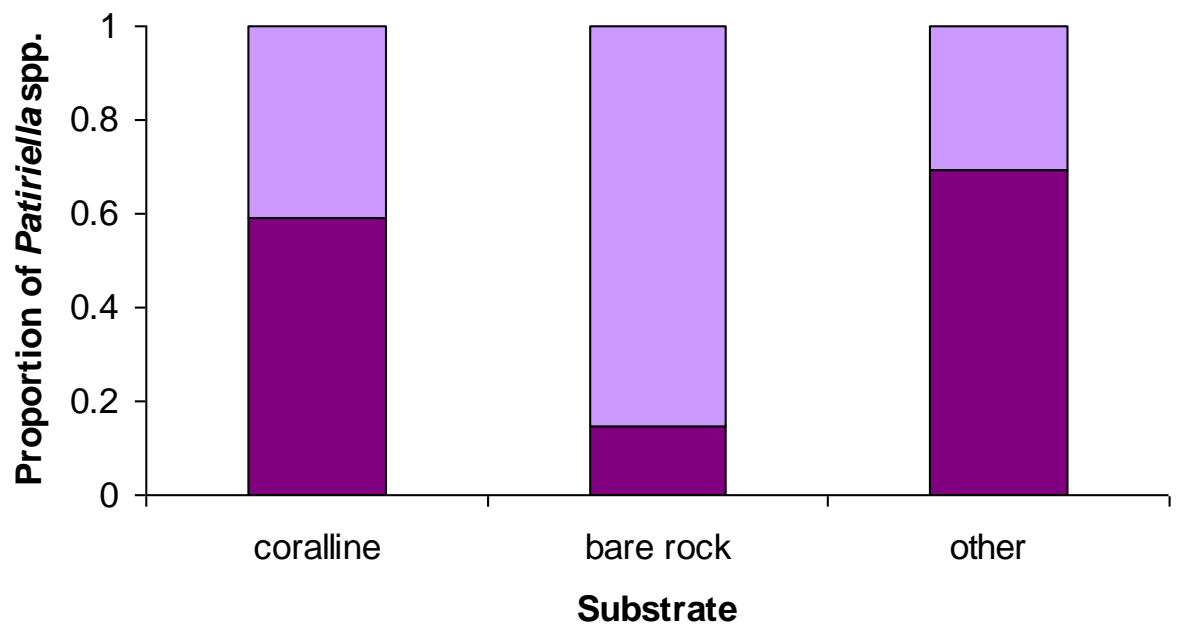

Figure 2.18: Proportion of Patiriella spp. on each substrate that were feeding (dark bars) and not feeding (light bars). Individuals on more than one substrate were excluded.

\section{Patiriella spp. Size}

Region had a marginally significant effect on the size of Patiriella spp. $\left(F_{1,344}=6.45, p=0.07\right)$, with a trend towards larger Patiriella spp. on the Wellington South Coast compared with those within Wellington Harbour (Figure 2.19).

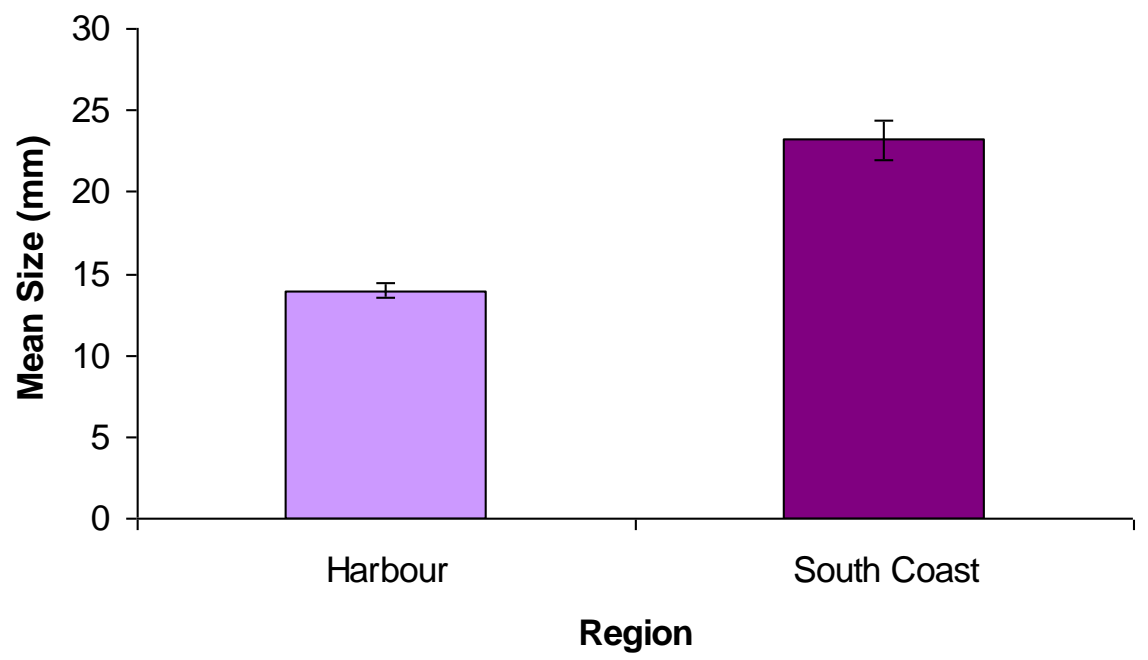

Figure 2.19: Mean size of Patiriella spp. $( \pm S E)$ in the Harbour and on the South Coast.

There appeared to be a larger spread of sizes on the South Coast, with an overall larger maximum size $(\max =53 \mathrm{~mm})$ than those in the Harbour $(\max =38 \mathrm{~mm})$ and a larger proportion of small individuals in the Harbour (Figure 2.20). 


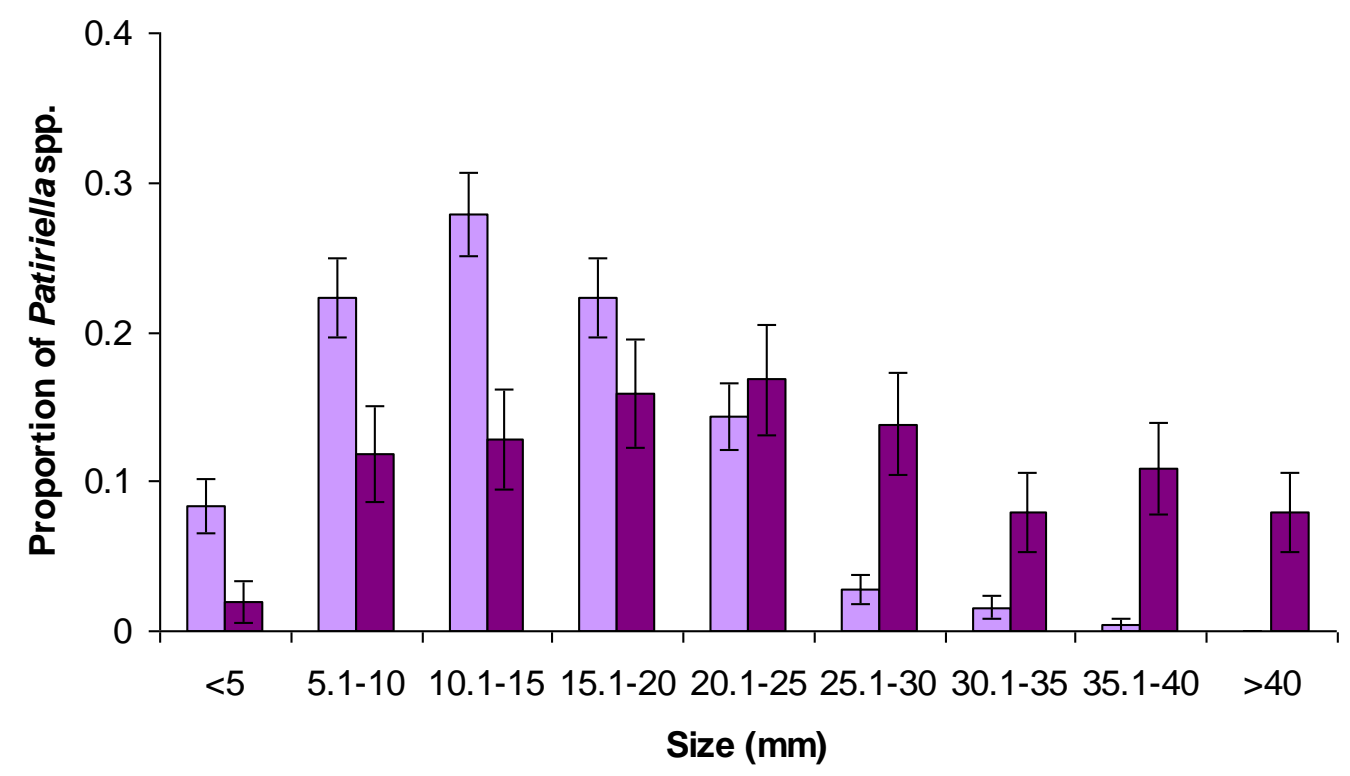

Figure 2.20: Distribution of Patiriella spp. sizes $( \pm S E)$ in the Harbour (light bars) and on the South Coast (dark bars).

\subsection{Discussion}

\section{Patiriella spp. Density and Distribution}

Although a variety of benthic invertebrates vary dramatically between Wellington Harbour and the Wellington South Coast (Phillips \& Hutchison, 2008), this does not appear to be true for Patiriella spp. There were no differences in the overall density of Patiriella spp. between Wellington Harbour and Wellington South Coast. However, there appears to be a greater density of Patiriella spp. on each cobble in the Harbour in August, compared with the South Coast and in other months. Patiriella spp. in the Harbour also occupied more cobbles, especially in August. Perhaps this difference between area and cobble density is due to the difference in maximum numbers per cobble between regions. Several cobbles with a large density of Patiriella spp. were found within the Harbour, but the same density per cobble was not found on the South Coast. This suggests there may be greater variation in Patiriella spp. numbers among cobbles than between regions or months. Chapman (2002b) found that species assemblages varied more at the scale of cobbles than among either shore level or site. 
Many intertidal species are over-dispersed within cobble fields, with many individuals on one cobble and many other cobbles with no individuals (Chapman, 2002b; 2005). The same trend was also found in this study, with only $18 \%$ of cobbles in the Harbour occupied by Patiriella spp. and $9 \%$ occupied on the South Coast. However, there were not a consistently large number of individuals per cobble, as the majority of occupied cobbles only contained one individual Patiriella spp. This could be due to the cobble size, which was limited to approximately $120 \mathrm{~mm}$ in this study. However, it has been shown that cobble size is not a significant predictor of species assemblages (Chapman, 2005). The reason for patchy distributions in cobble fields remains unclear. Chapman (2005) found that presence of other organisms or substrate under cobbles had little effect on species distributions. Previous studies have shown that the distribution of chitons was not correlated to cobble size or presence of other organisms, nor was it affected by algal cover (Grayson \& Chapman, 2004). However, the physical composition of cobbles may affect the assemblages that inhabit them. The urchin species Heliocidaris erythrogramma have been shown to prefer cobbles that were previously inhabited, suggesting a preference for substrate type (Smoothey \& Chapman, 2007). Semi-sheltered microhabitats, such as cavities within cobbles, provide the most diversity (Le Hir \& Hily, 2005) and perhaps explain some of the variation among cobbles. The surface complexity of cobbles has been found to affect the settlement of algal and sessile species on the top of cobbles, but it appears to have little effect on colonization by mobile species, or by sessile species on the underside of cobbles (McGuiness \& Underwood, 1986). Patiriella spp. larvae preferentially settle on the undersurface of substrata, with shade an important cue for settlement and metamorphosis (Byrne \& Barker, 1991). This suggests that shelter is an important habitat feature for Patiriella spp. settlement, but does not explain the distribution of mobile adults.

The presence of food resources may also affect the distribution of Patiriella spp. among cobbles. Patiriella exigua, an Australian species, feeds on microalgae (Jackson et al., 2009) and $P$. regularis appears to have a similar diet (Crump, 1971; Burgett, 1982). The distribution of the seastar Asterina 
pectinifera is affected by the availability of food, with translocated individuals moving back to areas with more abundant food resources (Kurihara, 1999). Many mobile grazers in intertidal cobble fields, including $P$. exigua, move away from cobbles at high tide and return during low tide (Cruz-Motta, 2005). This further supports the idea that Patiriella spp. can move to their preferred habitat. However, it is possible that they use cobbles as shelter during the low tide and leave to forage during high tide. Other seastar species have been found to migrate from deeper water to feed and then move back (Savy, 1987). Additional study into the features of cobbles preferred by Patiriella spp. along with their diel movements is required to better understand their habitat preference and distribution.

\section{Chiton Density and Distribution}

As with Patiriella spp., no consistent difference was found between the density of chitons within Wellington Harbour and the South Coast, either at the scale of unit area or per cobble. There were however, more cobbles occupied by chitons in the Harbour in August than at other times or regions. The reason for these differences, seen in both Patiriella spp. and chiton densities per cobble in August in the Harbour, is unknown. Weather is one possible explanation. On all days during the August survey the weather was calm, except for the days preceding the survey at Moa Point on the South Coast, when there were high winds and rain. Weather during November surveys was relatively calm, but with more rain than during August. Increased wave exposure caused by wind or changes in salinity caused by rain may have caused chitons and Patiriella spp. to seek refuge in deeper waters, leading to lower intertidal abundances for surveys during these times. More frequent surveys during periods of variable environmental conditions are required to determine whether weather events, such as heavy rain, can affect the movement and abundance of mobile intertidal grazers.

Chitons were more than twice as abundant as Patiriella spp., both within Wellington Harbour and on the South Coast. As with Patiriella spp. the distribution of chitons appears to be patchy, with only about $20 \%$ of cobbles 
occupied by chitons in either region, and most cobbles only had 1-2 chitons on them. Abundances of chitons were also found to be variable in New South Wales, Australia, where $74-99 \%$ of cobbles were unoccupied (Grayson \& Chapman, 2004). However, Grayson and Chapman (2004) also found that all but one species of chiton aggregated, with large numbers clustered on few cobbles (Grayson \& Chapman, 2004). This is in contradiction to the 1-2 chitons seen on most cobbles in this study. Grayson and Chapman (2004) also found that this distribution of chitons was not explained by cobble size, the presence of algae, or the presence of sessile or other mobile animals. Other studies on species assemblages under cobbles have found that cobble size, substrate and the presence of sessile organisms do not appear to affect colonization and therefore species diversity (Chapman, 2002b; 2005). Physical features of cobbles seem to be the most likely explanation for the distribution and diversity of species on cobbles (McGuiness \& Underwood, 1986; Smoothey \& Chapman, 2007).

This study found a negative correlation between the presence of Patiriella spp. and chitons on intertidal cobbles. As chiton distribution is not explained by the presence of other mobile species (Grayson \& Chapman, 2004) it is unlikely that chitons actively avoid Patiriella spp. However it is possible that Patiriella spp. actively avoid chitons. Branch and Branch (1980) found that $P$. exigua had less Cellana tramoserica (limpet) neighbours than expected and more conspecific neighbours, with $P$. exigua retreating from $C$. tramoserica. This suggests aggregation of $P$. exigua and avoidance of $C$. tramoserica. A similar relationship is possible between Patiriella spp. and chitons. Both species are abundant in intertidal cobble fields and both potentially compete for micro-algae (Crump, 1971; Burgett, 1982; Creese, 1988). Therefore, the negative correlation between these animals could be due to interference or exploitation competition. However, microhabitat may also be a determining factor in distribution, with Patiriella spp. and chitons having different preference for cobble features (Le Hir \& Hily, 2005).

Both Sypharochiton pelliserpentis and Chiton glaucus were the most abundant chitons within Wellington Harbour and the South Coast. Burgett 
(1982) also found that these species spanned the same regions as $P$. regularis. S. pelliserpentis was the most common chiton at South Coast sites, with $C$. glaucus the most common within the Harbour. The South Coast is an exposed region and therefore appears to be ideal for $S$. pelliserpentis which are abundant on exposed mainland shores (Creese, 1988). This species of chiton grazes on diatoms, cyanobacteria and other microalgae, while the largest component of $C$. glaucus' diet is coralline algae (Creese, 1988).

\section{Patiriella spp. Feeding}

Nearly half of Patiriella spp. on the Wellington South Coast were feeding, compared with only quarter feeding within Wellington Harbour. Therefore the hypothesis that more Patiriella spp. would be feeding within the Harbour, due to increased organic matter, must be rejected. Some seastars feed faster when in the presence of conspecifics (Gaymer et al., 2002); however, as densities are similar between the South Coast and the Harbour, this phenomenon does not explain the increased feeding frequency on the South Coast. Higher temperatures may also increase seastar frequency of feeding (Sanford, 1999), but water temperatures on the South Coast are generally lower than those within the Harbour (Helson et al., 2007). Food resources may be the likely cause of differences in feeding frequency. There may be lower quality food available to Patiriella spp. on the South Coast, and therefore they may need to feed more. Or increased wave exposure may increase the accessibility of food resources on the South Coast. Alternatively, food resources may be the same in both regions, but chemodetection may be better on the South Coast. The South Coast is more exposed and therefore has greater wave action, which has been shown to increase the movement of seastars towards their prey (Gagnon et al., 2003).

Patiriella spp. feed by everting their stomach through their mouth to cover the substrate and any organic material trapped beneath is digested by enzymes (Crump, 1971). Previous studies have examined the substrate upon which Patiriella spp. have their stomachs everted as an indication of their food items. These food items include micro-organisms, such as detritus and 
diatom films, and macro-organisms, such as carcasses and fragments of crabs, fish (Crump, 1971), mussels (Witman \& Grange, 1998) and crustose coralline algae (Burgett, 1982). The findings of this study support the observations that the seastars consume macro-organisms, as individuals on crustose coralline algae or other visible substrate were observed to be feeding more frequently than those on apparently bare rock. A few Patiriella spp. were also observed feeding upon crab legs and dead abalone (Haliotis iris). This is consistent with Crump's (1971) conclusion that $P$. regularis feed mostly on algae and micro-organisms, but require supplementation of carrion for growth. It is still unknown whether Patiriella spp. feed on crustose coralline algae or micro-organisms within the algae. Patiriella exigua have been found to have a large effect on the temporal and spatial distribution of micro-algal food (Jackson et al., 2009). Feeding in Patiriella spp. appears to be similar and could therefore potentially affect other grazers, such as chitons, which have overlapping diets.

\section{Patiriella spp. Size}

Patiriella spp. at South Coast sites were marginally larger than Patiriella spp. at Harbour sites. Crump (1971) suggested that higher densities in an area may result in greater competition for food and therefore cause smaller sizes. However, the hypothesis that reduced sizes are due to increased densities in the Harbour cannot be accepted, as densities were not significantly different between the regions. As feeding is also more frequent on the South Coast, it is possible that feeding rate and size are related. Perhaps greater abundance of food or better chemodetection of food on the South Coast, due to wave action (Gagnon et al., 2003), increases feeding and leads to greater sizes of individuals. Alternatively, size distributions may be related to recruitment, size-selective mortality, movement or a combination of these.

The majority of Patiriella spp. surveyed at all sites in this study were small, with an average of $23 \mathrm{~mm}$ on the South Coast and an average of only $14 \mathrm{~mm}$ in the Harbour. Burgett (1982) observed that $P$. regularis smaller than $29 \mathrm{~mm}$ did not have gonads and therefore assumed individuals smaller than this 
were sexually immature juveniles. However, Crump (1971) found that in areas with small $P$. regularis, individuals attained mature gonads after being supplemented on abundant food supplies and concluded that they were nutritionally deprived adults and not immature juveniles. It is not clear whether the Patiriella spp. in this study were adults or juveniles, but larger individuals have been seen in deeper water outside of the intertidal area of this study (S. Palmer, pers. obs.). At Leigh Marine Reserve, individuals from metamorphosis up to $15 \mathrm{~mm}$ were observed under cobbles in the intertidal, with larger 15-60mm Patiriella spp. seen on the open reef flat (Burgett, 1982). Other animals also show partitioned size classes, with small individuals high in the intertidal and successively larger individuals further down the shore and into the sublittoral fringe (Creese, 1988).

\section{Summary}

Patiriella spp. densities, as well as chiton densities, were similar between Wellington Harbour and Wellington South Coast, although patterns were dependent upon the sampling month. Spatial scale of observation appears to be important, with a trend towards more Patiriella spp. per cobble in the Harbour in August, compared with per area. A longer study is required to determine whether there is a significant density pattern among months of the year and among years. Both Patiriella spp. and chiton populations are patchy, with many unoccupied cobbles. The distribution of Patiriella spp. and chitons on cobbles in cobble fields is most likely to be caused by either the availability of food resources, shelter from environmental conditions, interference competition or predation.

Patiriella spp. and chitons are both abundant at all sites surveyed and are therefore likely to be common throughout the Wellington region. There was a negative correlation between these two taxa at the level of cobbles and this could indicate avoidance of chitons by Patiriella spp., due to interference competition. However, a preference for differing microhabitats would also explain this segregation. 
Patiriella spp. on the Wellington South Coast tended to be larger than those within the Harbour and were also feeding more frequently. This could be a sign of differences in food resources between the two regions, or better detection of food by Patiriella spp. due to increased wave action. Limited food resources have been found to decrease growth, but not affect mortality (Crump, 1971) and so could explain why there were constant densities between regions, but differences in feeding and size. Patiriella spp. individuals within the surveyed intertidal cobble fields were relatively small, suggesting individuals possibly migrate to deeper waters as they grow.

Patiriella spp. appear to feed mostly on micro-organisms, but supplement their diet with carrion. They may have an effect on community dynamics by decreasing coralline algal cover, although it is still unclear whether this is a targeted food source or just an artefact of feeding on micro-organisms within the algae. 


\section{3}

\section{Scavenging Behaviour of Patiriella spp.}

\section{Abstract}

Scavengers feed on an unpredictable carrion supply and therefore must often survive for long periods without food. Detection of carrion varies among species and is affected by strength and direction of current, preferred prey species, freshness of carrion and tidal flow. Hunger levels affect how scavengers respond to detected carrion. The aim of this chapter was to investigate behaviour of the seastar Patiriella spp. when carrion is available. Field experiments used mussel carrion bait to observe the numbers and species of scavengers that move towards bait within Wellington Harbour and on the Wellington South Coast. Laboratory experiments examined the response of Patiriella spp. to mussel carrion at varying states of hunger and also compared the responses between Patiriella spp. collected from Wellington Harbour and the Wellington South Coast. Four species were consistently attracted to bait in the field: two species of whelk (Cominella virgata and Cominella maculosa); Patiriella spp.; and a crab (Heterozius rotundifrons). In laboratory experiments, prior food supply had a significant effect on the responses of Patiriella spp. to carrion bait, with previously unfed seastars moving towards carrion and feeding more than those previously supplemented with carrion. Both field and laboratory experiments found no significant differences between the responses of Patiriella spp. from Wellington Harbour and responses of Patiriella spp. from the South Coast. The responses of Patiriella spp. from both regions were similar to the 
responses of unfed seastars, which suggests that carrion supply is limiting in both the Wellington Harbour and on the Wellington South Coast.

\subsection{Introduction}

Scavengers are relatively common, with representatives in most major phyla (Britton \& Morton, 1994). Due to the unpredictable and infrequent supply of carrion (Britton \& Morton, 1994) most scavengers are facultative, capable of obtaining nutrition from alternative sources. The irregular occurrence of dead organic matter and its difficulty to locate means scavengers must often survive long periods without food (Stenton-Dozey et al., 1995). For example, some scavenging marine gastropods (e.g., Babylonia lutosa, Nassarius obsoletus, Nassarius festivus and Hemifuscus tuba) can survive more than 100 days of starvation (Curtis \& Hurd, 1979; Morton, 1986; 1990). Most previous studies examining marine scavengers have concentrated on the role and behaviours of marine gastropod scavengers (Morton \& Britton, 2003; Morton \& Jones, 2003; Morton, 2006).

Detection of, and attraction to, carrion is required for animals to be effective scavengers. Marine scavengers have varying abilities to detect carrion, which is often dependent upon: 1) strength and direction of current (Rochette et al., 1994), with upstream carrion detected more easily than downstream carrion due to the transport of odours towards the scavenger (Morton \& Britton, 2003); 2) preferred prey species; 3) whether carrion is fresh or decayed (Stenton-Dozey et al., 1995); and 4) tidal flow, with reduced water flow at low tide decreasing the distribution of chemical cues which indicate the presence of dead or decaying matter (Morton \& Britton, 2003). Seastars have an ideal shape for chemosensory perception, as they are able to receive chemical cues from all directions equally (Brewer \& Konar, 2005). Some seastars use concentration differences as measured at the tip of different arms to guide orientation to food (Rochette et al., 1994). Seastars, such as Pycnopodia helianthoides (Rochette et al., 1994) and Asterias forbesi (Moore \& Lepper, 1997) have been found to use chemoreceptive abilities to quickly find and consume dead or damaged prey items. Movement towards prey or carrion is 
dependent on hunger levels, with well-fed seastars often showing erratic movement, but starved individuals moving predictably towards a possible food source (Rochette et al., 1994).

Although the seastar Patiriella regularis feeds primarily on algal or microbial biofilms (Burgett, 1982), they also gain a large portion of their nutrition by scavenging on dead and decaying matter (Crump, 1971). In laboratory experiments, $P$. regularis gained more weight when fed a diet of crab carrion than when fed a diet of fish carrion (Crump, 1971). Patiriella regularis have also been known to feed on live mussels, but it takes at least 15 hours for 3-8 seastars to consume the viscera (Witman \& Grange, 1998). Therefore, consumption of dead or decaying animals would require less time and energy, but potentially provide the same nutrition, as live animals. In addition to the identity of carrion, the presence of conspecifics or heterospecifics may influence the attraction and feeding of seastars on carrion. For example, intraspecific interactions in the seastars Asterias vulgaris and Leptasterias polaris increased feeding rates, while interspecific interactions between these species decreased feeding rates (Gaymer et al., 2002). The presence of heterospecifics and conspecifics may also trigger either an avoidance or aggregative response (Gaymer et al., 2002). Avoidance may result in dispersion (Sloan \& Northway, 1982) which reduces competitive effects, but feeding often takes precedence over conspecific avoidance. The ability to arrive first at a carrion food source is also likely to be an advantage (Morton \& Britton, 2003). The feeding of Patiriella can be affected by interspecific competition. Patiriella exigua caged with the limpet Cellana tramoserica had decreased growth in grazing field experiments (Arrontes \& Underwood, 1991). However, the effect of the presence of conspecifics and heterospecifics on scavenger feeding by Patiriella spp. is currently unknown.

Many marine scavengers appear to feed to satiation before leaving the food source, which suggests that time spent feeding can be used as an indication of an individual's hunger, but this response varies among species. StentonDozey et al. (1995) found that the scavenging gastropod Bullia digitalis fed for longer after starvation, due to a decreased gut threshold, even though its 
meal size remained the same. Morton (2006) found that Ergalatax contractus reached satiation faster after starvation in order to quickly restore its energy balance. Starvation can also have other consequences, with hunger affecting whether or not the gastropod Nassarius siquijorensis seeks food (Morton \& Chan, 1999) and increasing the speed of arrival at bait for starved Ergalatax contractus (Morton, 2006). Hunger of some scavengers can also override the risk of predation. Nassarius spp. were found to avoid carrion that included crushed conspecifics, unless they were starved for an extended length of time (Morton \& Chan, 1999; Morton \& Britton, 2003). Morton and Chan (1999) found that well-fed scavengers were more active than starved animals. The availability of carrion also significantly affects growth, with supplemental carrion resulting in faster growth and larger individuals of the intertidal scavenger Nassarius dorsatus in field experiments (McKillup \& McKillup, 1997).

The first aim of this chapter is to identify whether Patiriella spp. are attracted to carrion in the field and to also identify what other species are attracted to, and possible competitors for, the same carrion. I hypothesised that, as scavengers, Patiriella spp. would be attracted to bait. I also predicted that there would be a negative correlation between the number of Patiriella spp. and the number of the other most dominant scavenger species at carrion food, due to the avoidance of heterospecifics.

The second aim was to investigate the effect of diet on the response of Patiriella spp. to carrion. I hypothesised that starved Patiriella spp. would move faster towards carrion and would remain feeding on it for longer, than well-fed Patiriella spp. It was also hypothesised that Patiriella spp. from Wellington South Coast populations would show greater responses to carrion than Harbour populations. This is due to the smaller quantity of organic matter available on the South Coast (Helson et al., 2007), which may result in a poorer diet for Patiriella spp. on Wellington's South Coast. 


\subsection{Methods}

\section{Scavenger Field Experiments}

During January and February 2009, experiments were conducted to investigate the attraction of Patiriella spp. to carrion bait stations at six sites: three sites within Wellington Harbour (Point Howard, Shelly Bay and Point Halswell); and three sites on the South Coast (Moa Point, Island Bay and the Quarry; see Figure 2.1). At each site, there were rocky outcrops interspersed with patches of cobbles and the study was conducted within the cobble areas only. The experiment was carried out on a different day for each site, but sites were assigned randomly to days, and alternated between the South Coast and Harbour.

On each day, 50 blue mussels (Mytilus galloprovincialis) were collected to be used as bait. Mussels were approximately $60 \mathrm{~mm}$ in length and were always collected from the same site (Shelly Bay) to minimise bias due to possible differences in the mussels among sites. Collections were always made immediately prior to each experiment.

At the field site, five bait stations were placed just below the waterline and monitored every 30 minutes for two hours. Each bait station was created by placing 10 mussels inside a white cotton sock, tying a knot in the sock and then crushing the mussels. Each bait station was created immediately prior to placement in the water and the time of placement was recorded. Bait stations were positioned at least five metres away from each other along the shoreline and were secured with cobbles (Figure 3.1).

Each bait station was removed from the water approximately 30 minutes after placement and all animals attached to the baited sock were placed in an empty container. Any animals that fell off the baited sock during the removal were also recovered and placed in the container. The baited sock was then returned to the water. Where possible the bait station was placed in the same position, but in some cases it had to be moved up or down the shore to 
ensure that it remained submerged in shallow water as the tide came in and then receded. All experiments were commenced approximately one hour before low tide and completed approximately one hour after low tide.

All animals removed from a bait station were counted and identified to species where possible. All Patiriella spp. individuals removed from bait stations were measured from the tip of the longest arm to the opposite armpit (see Figure 2.4). The time at which animals were removed from the bait station was recorded, along with the bait station number. Once counted, all animals were held in a container of seawater to prevent them from being included in subsequent counts.

The removal of bait stations, recording of animals and replacement of bait stations was repeated every 30 minutes, for a total of four times for each station. The experiment was carried out in parallel for all bait stations at a site. At the conclusion of the experiment all baited socks were removed and all animals were returned to the sea. New socks were used at each site.

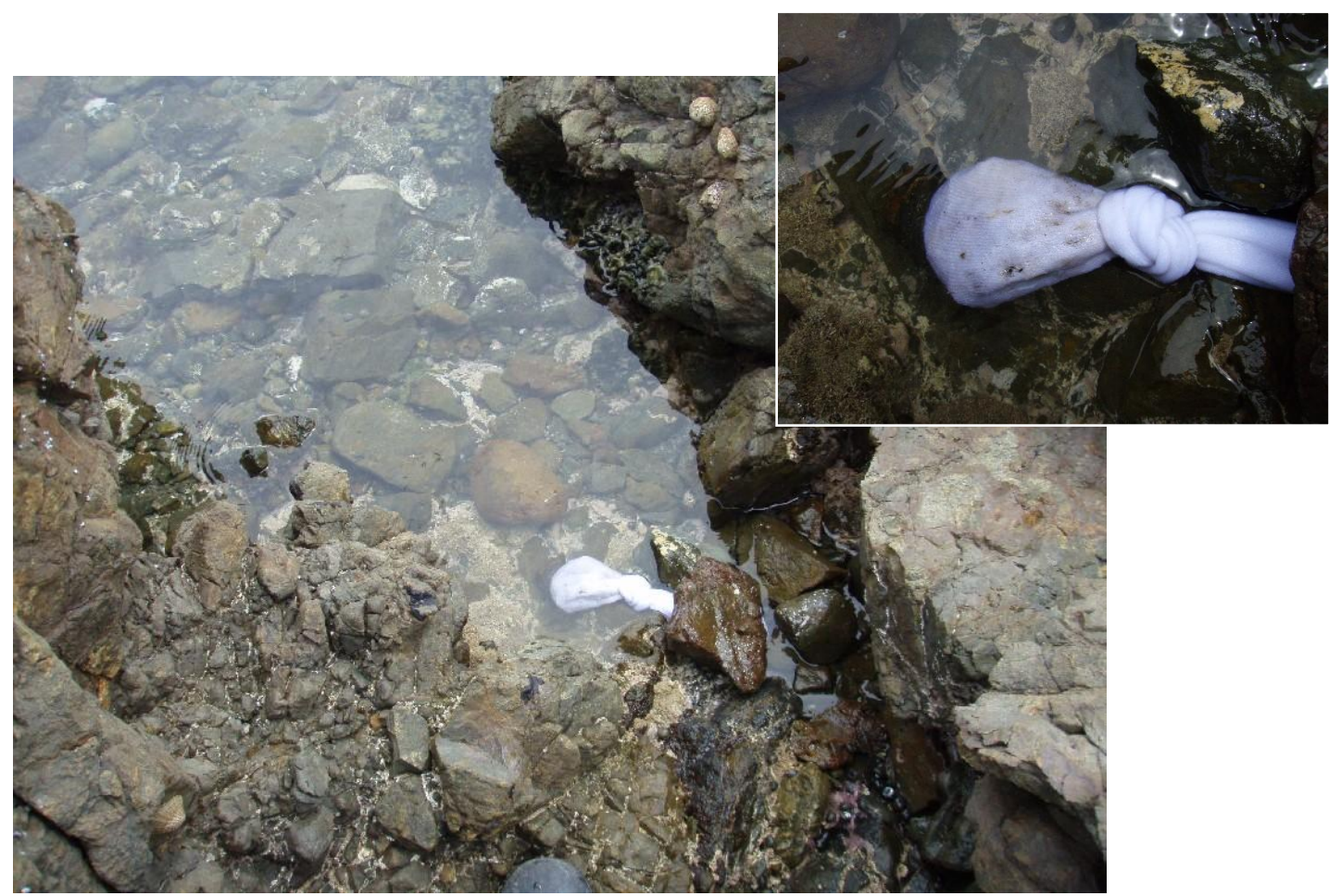

Figure 3.1: Bait station placed below waterline at Moa Point 


\section{Scavenger Laboratory Experiments}

Two sets of laboratory experiments were conducted to investigate the response of Patiriella spp. to supplemental carrion food. The first experiment was used to determine whether starved Patiriella spp. had a greater attraction to supplemental food than well-fed Patiriella spp. The second experiment examined if there was a difference in the attraction to carrion by Patiriella spp. from Wellington Harbour versus Wellington South Coast.

\section{Unfed Versus Supplemented}

Patiriella spp. were collected from Shelly Bay (sizes ranged from 15-40mm) and randomly assigned to one of three food treatments: 1) supplemented;2) non-supplemented; and 3) unfed (Figure 3.2). Three 50-litre aquaria were used for each treatment to allow for variation among aquaria. Each of the aquaria was randomly assigned a position on a seatable and had a constant flow of unfiltered seawater, which was independently piped into each aquarium. Each aquarium contained 10 Patiriella spp. individuals $(n=30$ individuals for each treatment). The supplemented and non-supplemented treatments contained cobbles, collected from Wellington Harbour, that were at least partly covered with crustose coralline algae and cobbles were also a possible source of micro-organisms. Crushed mussels were added to supplemented treatments every two days. Initially, two mussels were placed in supplemented treatments and then subsequent supplementation consisted of removing the oldest mussel, which was replaced with a freshly crushed mussel. Mussels were never depleted in the supplemented treatment and therefore there was an unlimited supply of food for these Patiriella spp. The non-supplemented treatment did not receive any form of supplementation and only the crustose coralline algae and micro-organisms were available as a possible food source. The unfed treatment did not contain any cobbles or supplementation, but as unfiltered seawater constantly flowed through the aquaria, this is likely to have supplied some micro-organisms.

Prior to placement in aquaria, each Patiriella spp. was weighed and measured. Measurements were done from the tip of the longest arm to the 
opposite armpit (see Figure 2.4). Wet weights of individuals were taken after first blotting excess water from each seastar. Patiriella spp. were then held in the treatments for one week and again weighed and measured prior to testing for attraction to supplemental food (see below).
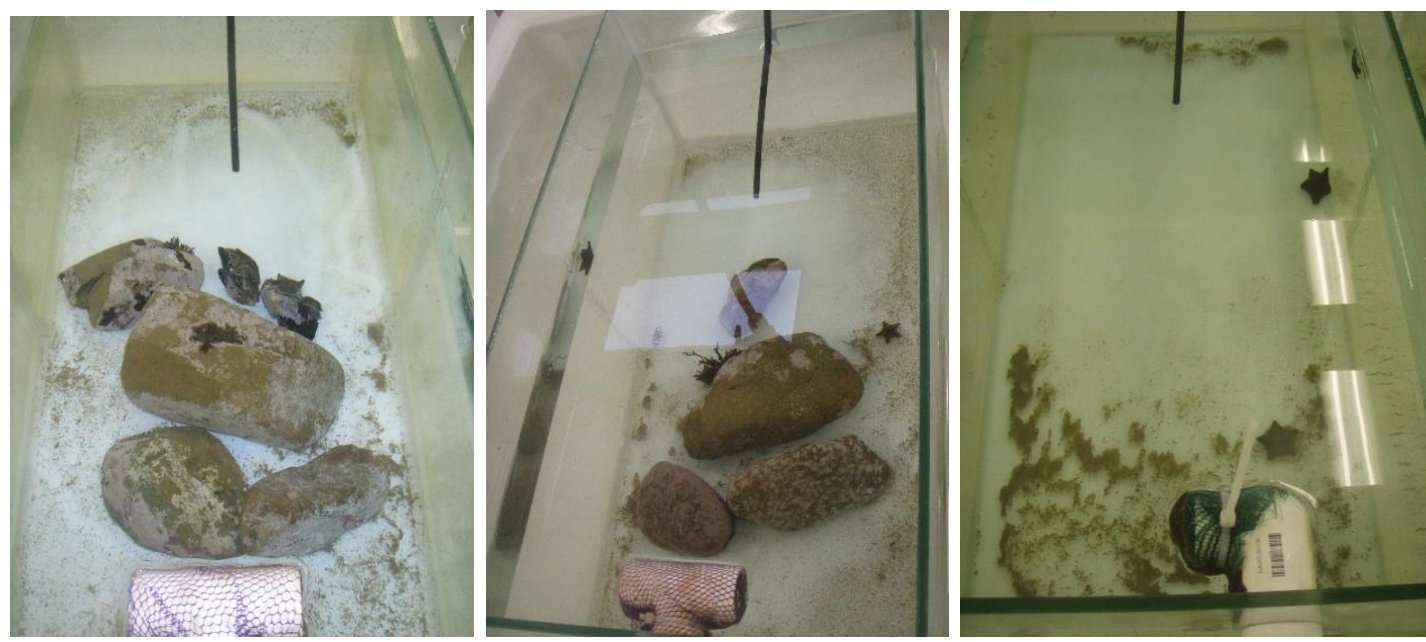

Figure 3.2: Aquaria holding supplemented, non-supplemented and unfed treatments (from left to right) prior to testing for supplemental food attraction.

To test for attraction to carrion, a single Patiriella spp. was placed in a 50 litre aquarium containing only flowing unfiltered seawater. After allowing the seastar to acclimatise for 10 minutes a crushed mussel was added to the aquarium at a distance of $30 \mathrm{~cm}$ from the seastar. The time taken for the Patiriella spp. to make first contact with the mussel was recorded, as well as the time that the Patiriella spp. stayed in contact with the mussel. The seastar was left for 60 minutes and if no contact was made with the mussel during this time it was removed. If the seastar contacted the mussel it was left for a further 30 minutes and was recorded as feeding if its stomach was extruded at the end of this time. This was repeated for all 30 Patiriella spp., which were selected in random order, with at least one replicate from each treatment run simultaneously.

\section{Harbour Versus South Coast}

Twelve Patiriella spp. were collected from each of the three Wellington Harbour sites and three Wellington South Coast sites. All collections were done from within intertidal cobble fields during low tide (sizes were 15$40 \mathrm{~mm}$ ). Each seastar was subjected to the same experimental design as 
described above, to test for differential attraction to carrion between the regions. Testing was done over three days with Patiriella spp. from one Harbour site and one South Coast site collected and tested each day. This was done to minimise the possible effect of collection day on the comparison between Harbour and South Coast animals. Tests were done on the same day as the individual was collected to minimise the effect of being held in the laboratory. Again, time to first contact was recorded, along with the time feeding on the mussel and whether the stomach was extruded at the end. Three replicates from each of the Harbour and South Coast sites were run simultaneously.

\section{Statistical Analysis}

Data were analysed using JMP 4.0.4. ANOVAs were run using the EMS, but these results were compared to REML procedure and in all cases results were qualitatively the same. All figures show untransformed data for ease of interpretation. Levene's test was used to test the assumption of homogeneity of variance and Shapiro-Wilk's was used to test for normality.

\section{Field Data}

The numbers of Patiriella spp. attracted to bait stations on the Wellington South Coast was not normally distributed, but distribution was normal for Wellington Harbour and whelk data. Variances were equal for all field data and so analysis continued with untransformed data as ANOVA is robust to deviations from normality.

Mixed model ANOVA was performed to compare the number of Patiriella spp. attracted to bait stations between the regions of Wellington Harbour and the Wellington South Coast. The full model consisted of one factor of region (fixed, 2 levels) and one factor of site nested within region (random, 3 levels per region).

An ANCOVA was run to test for an effect of whelk numbers on Patiriella spp. numbers at each bait station, each time organisms were removed. Region 
was used as a factor (fixed, 2 levels). All whelk species were analysed together as a single variable.

\section{Laboratory Data}

The average weight gain of Patiriella spp. in feeding treatments was calculated per tank after one week. The weight gain of individuals could not be determined as individual seastars were not identifiable. Weight gain data was normally distributed with equal variances. One-way ANOVA was used to compare the weight gain among treatments. The model was run with one factor of treatment (fixed, 3 levels). A Tukey post-hoc test was run to determine which treatments were significantly different. A matched pairs Ttest was also run to assess whether there was significant weight gain or loss in each of the feeding treatments.

Chi-square analysis was used to determine whether there was a difference among treatments for the number of Patiriella spp. actively feeding and the number of Patiriella spp. that contacted a mussel.

One-way ANOVA was performed on the time for Patiriella spp. to make first contact with the mussel and also on the time that Patiriella spp. stayed in contact with the mussel. Patiriella spp. that did not contact the mussel at all were excluded. This analysis was run for the starved versus well-fed experimental data, with one factor of treatment (fixed, 3 levels) and also for the Harbour versus South Coast data, with one factor of region (fixed, 2 levels).

Data on the time spent in contact with the mussel, for different feeding regimes, was not normally distributed and neither was the time taken to first contact the mussel, for regional treatments. However, variances were equal for these data sets and so ANOVA was performed on untransformed data. The data of time spent on the mussel for Patiriella spp. from different regions, was not normally distributed and did not have equal variances and therefore the nonparametric Kruskal-Wallis test was used for analysis of this. Data for 
time taken for Patiriella spp. to contact the mussel and time spent in contact with the mussel excludes Patiriella spp. that did not contact the mussel at all and therefore sample sizes are small and these results should only be taken as an indication.

\subsection{Results}

\section{Scavenger Field Experiments}

\section{Harbour Versus South Coast}

There was no significant difference in numbers of Patiriella spp. attracted to bait stations in Wellington Harbour compared with bait stations on the Wellington South Coast $\left(F_{1,24}=0.53, p=0.51\right)$, although there was a slight trend towards more Patiriella spp. in Wellington Harbour (Figure 3.3). No Patiriella spp. arrived at five of the 30 bait stations.

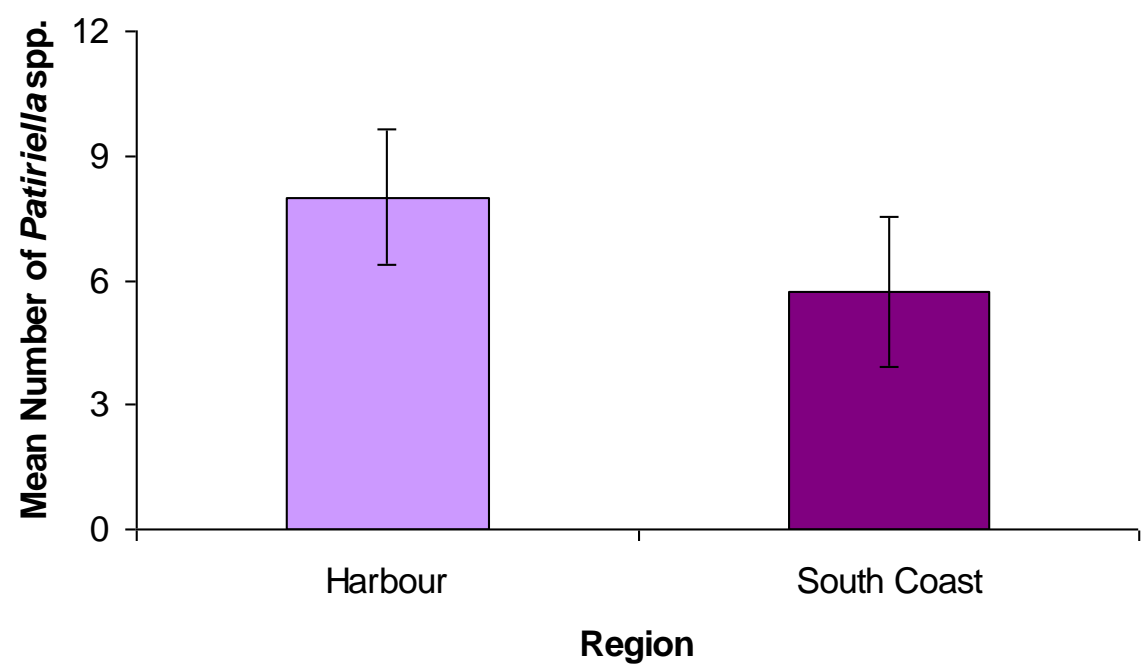

Figure 3.3: Mean number of Patiriella spp. arriving at bait stations ( \pm SE) over a two-hour period at Wellington Harbour sites and at Wellington South Coast sites.

\section{Other Species}

The species found on bait stations were dominated by four species: two whelks (Cominella virgata and Cominella maculosa), Patiriella spp. and a crab (Heterozius rotundifrons; Table 3.1). A few other seastar and crab species were present, but their total numbers were low. 
Table 3.1: Average number of the most abundant species ( $\pm S E$ ) per bait station in Wellington Harbour and on the Wellington South Coast.

\begin{tabular}{llcc}
\hline Species & Common Name & Harbour & South Coast \\
\hline Cominella virgata & Red-mouthed whelk & $118( \pm 20)$ & $76( \pm 18)$ \\
Cominella maculosa & Spotted whelk & $7( \pm 2)$ & $11( \pm 2)$ \\
Patiriella spp. & Cushion star & $7( \pm 2)$ & $6( \pm 2)$ \\
Heterozius rotundifrons & Big-handed crab & $3( \pm 1)$ & $5( \pm 2)$ \\
\hline
\end{tabular}

The number of whelks did not have a significant effect on the number of Patiriella spp. at each bait station $\left(\mathrm{F}_{1,117}=0.01, \mathrm{p}=0.91\right)$ and the number of Patiriella spp. was not significantly different between regions $\left(F_{1,117}=0.77\right.$, $p=0.38$; Figure 3.4).

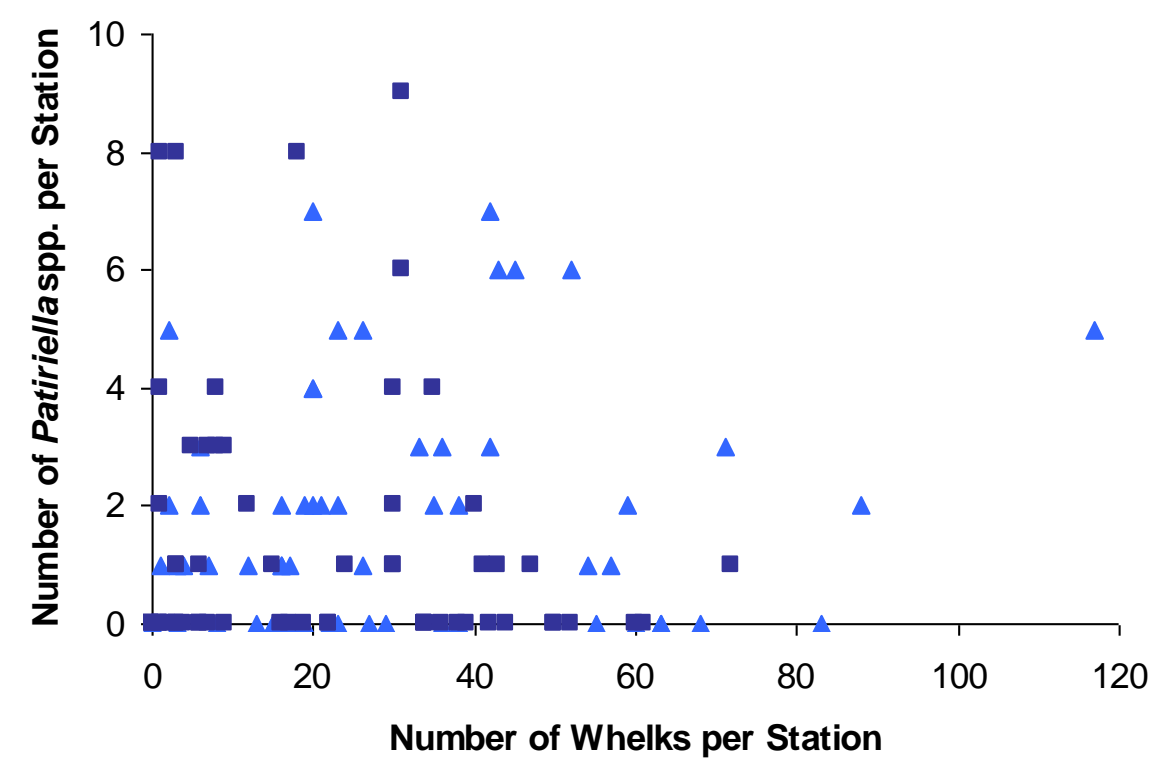

Figure 3.4: Relationship between the number of Patiriella spp. and the number of whelks ( $C$. virgata and $C$. maculosa combined) on each bait station, at each observation/removal period, in Wellington Harbour (light triangles) and on the Wellington South Coast (dark squares).

\section{Scavenger Laboratory Experiments}

\section{Unfed Versus Supplemented}

There was a significant difference in the average weight gain of seastars, per aquarium, among treatments $\left(\mathrm{F}_{2,7}=24.53, \mathrm{p}<0.01\right)$. Seastars in the supplemented treatments showed significantly more weight gain after one week than seastars in either the unfed or non-supplemented treatments 
(Figure 3.5). The end weight of seastars in supplemented treatments, averaged per aquarium, was significantly greater than their average start weights $(t=5.80, p=0.03)$, while there were no significant differences between the start and end weights of seastars in the unfed ( $t=-2.91, p=0.10)$ or nonsupplemented ( $\mathrm{t}=-1.79, \mathrm{p}=0.22)$ treatments. There was a trend towards weight loss in both unfed and non-supplemented treatments (Figure 3.5).

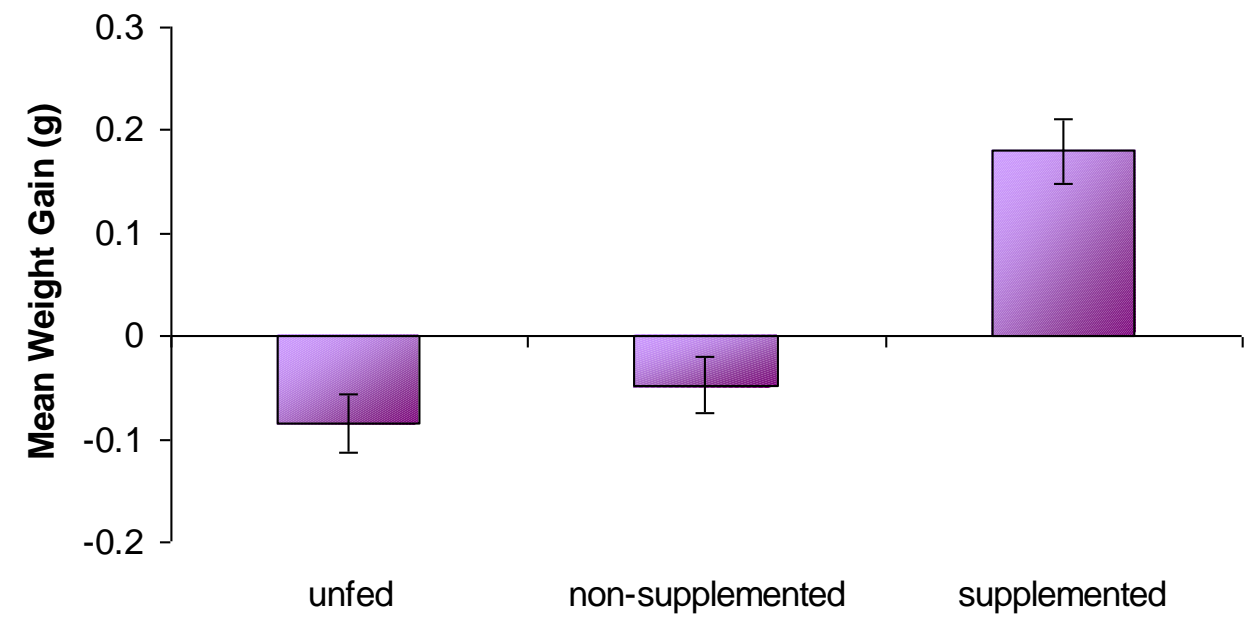

Feeding Treatment

Figure 3.5: Mean weight gain of Patiriella spp. ( $\pm S E)$ after one week in unfed, nonsupplemented and supplemented treatments.

Seastars from the three treatments responded differently when offered carrion. Fewer seastars from the supplemented treatments were feeding at the end of experiments, compared with seastars from either the unfed or nonsupplemented treatments $\left(\chi^{2}=7.92, n=90, p=0.02\right.$; Figure 3.6). Of those feeding, all were in contact with the mussel, except for two Patiriella spp. from the unfed treatment, which were feeding even though not on the mussel.

Fewer seastars from the supplemented treatment contacted the mussel than from either the unfed or non-supplemented treatments $\left(\chi^{2}=9.26, n=90\right.$, $p=0.01$; Figure 3.7). 


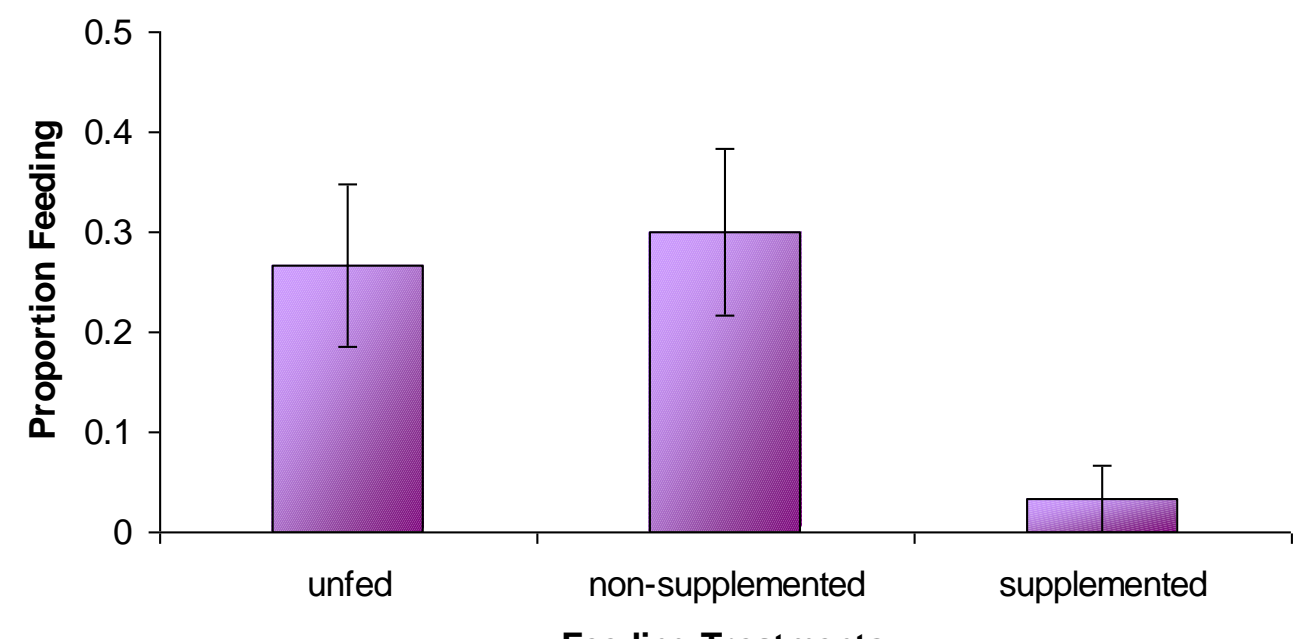

Feeding Treatments

Figure 3.6: Proportion of Patiriella spp. ( $\pm S E)$, from different week-long feeding regimes, which were feeding after being offered carrion.

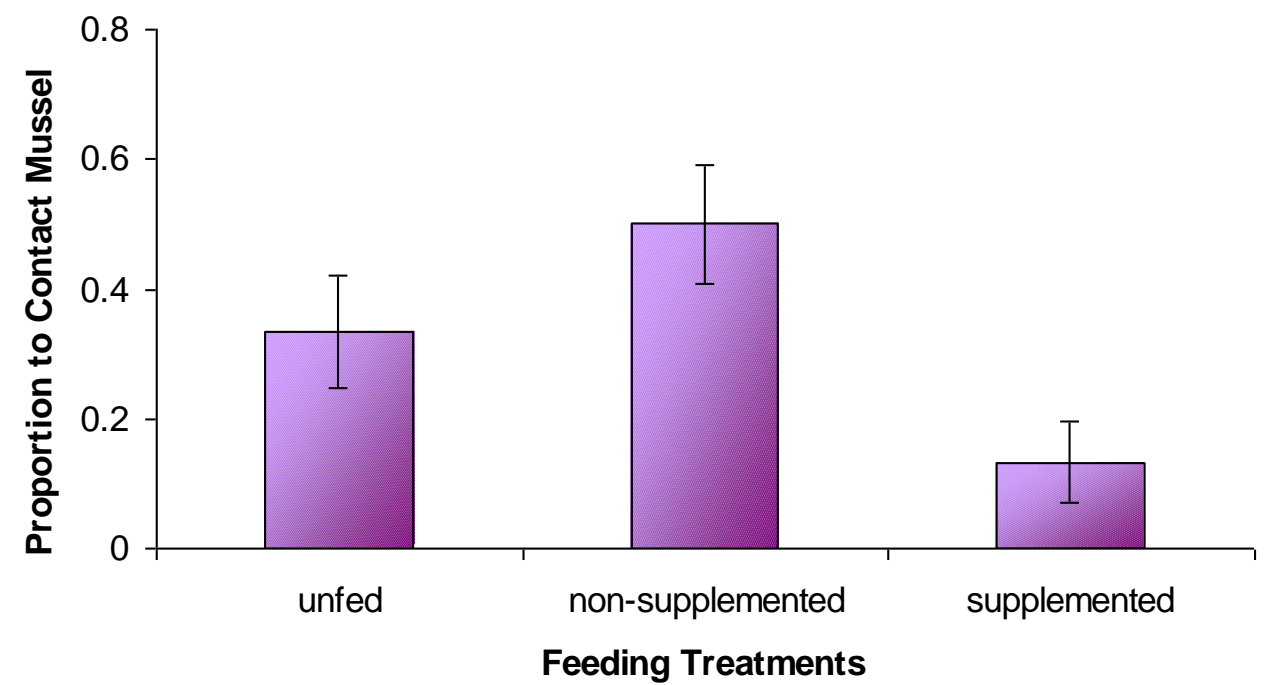

Figure 3.7: Proportion of Patiriella spp. ( $\pm \mathrm{SE})$, from different week-long feeding regimes, which came into contact with a mussel.

Of those Patiriella spp. that did contact the mussel, there was no significant difference among treatments on the time it took for seastars to come into first contact with the mussel $\left(\mathrm{F}_{2,26}=0.32, \mathrm{p}=0.72\right)$. For all treatments the average contact time was more than 30 minutes.

Of all Patiriella spp. that contacted the offered mussel, an average of $80 \%$ $(\mathrm{SE}=13)$ of unfed seastars, $60 \%(\mathrm{SE}=13)$ of non-supplemented seastars and 
$33 \%(\mathrm{SE}=23)$ of supplemented seastars stayed in contact with the mussel for the maximum time of 30 minutes. There was no significant difference among treatments in the time that Patiriella spp. stayed in contact with the mussel $\left(F_{2,26}=2.03, p=0.15\right)$; although there was a trend for unfed seastars to stay on the mussel the longest, followed by non-supplemented seastars, with supplemented seastars staying on the offered mussel for the shortest time (Figure 3.8).

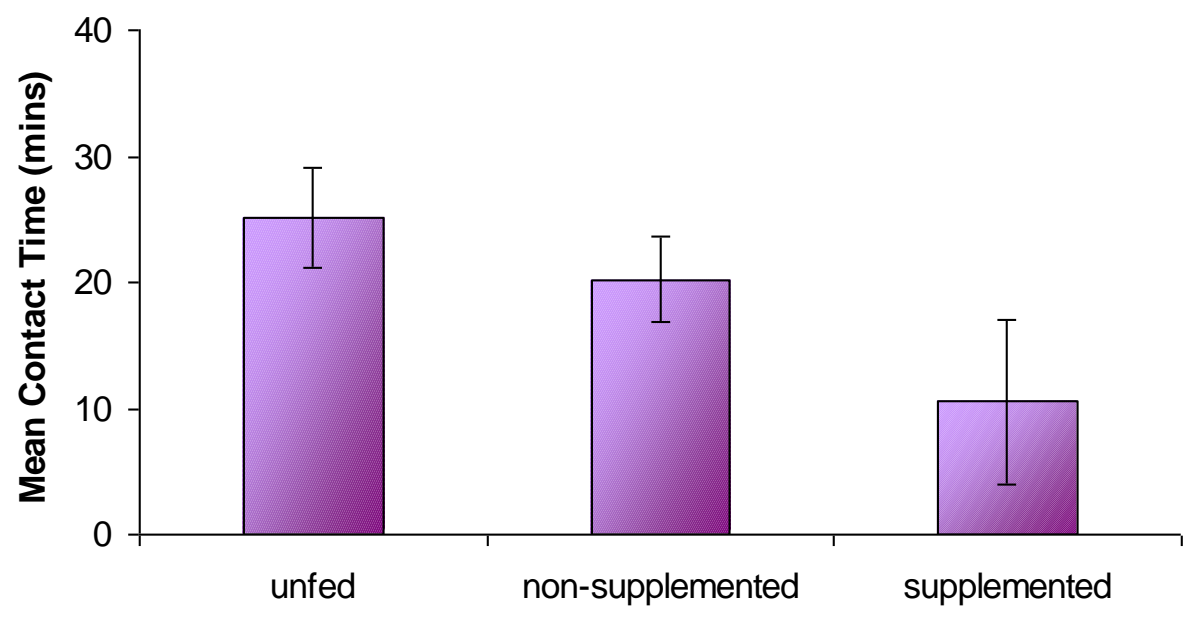

Feeding Treatment

Figure 3.8: Mean time that Patiriella spp. stayed in contact with a mussel after first contact $( \pm \mathrm{SE})$ for varying week-long feeding regimes. Excludes Patiriella spp. that did not contact a mussel at all.

\section{Harbour Versus South Coast}

The originating region had no effect on the proportion of Patiriella spp. feeding at the end of experiments $\left(\chi^{2}=0.00, n=72, p=1.00\right)$. Exactly the same number of Patiriella spp. from Wellington Harbour were feeding as those feeding from the Wellington South Coast (Figure 3.9).

There was no significant effect of region on the proportion of Patiriella spp. to come into contact with the offered mussel $\left(\chi^{2}=2.36, n=72, p=0.13\right)$, although there was a trend towards more contact by Patiriella spp. from the Wellington Harbour, compared with the Wellington South Coast (Figure 3.10). 


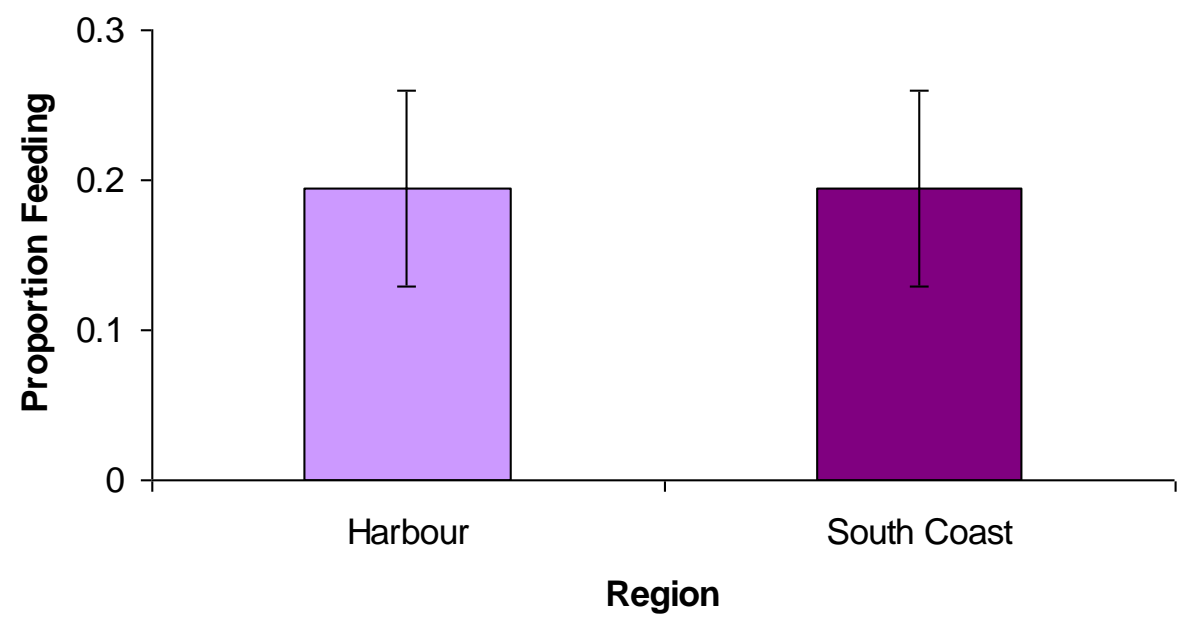

Figure 3.9: Proportion of Patiriella spp. $( \pm S E)$, from the Wellington Harbour and the Wellington South Coast, which were feeding at the end of the experiments.

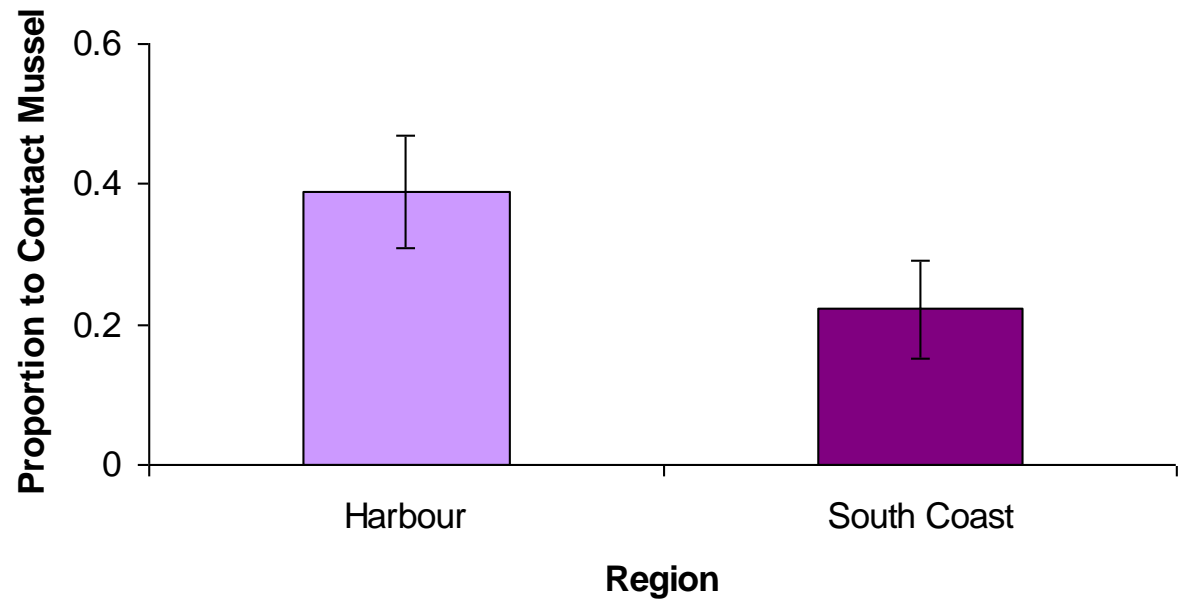

Figure 3.10: Proportion of Patiriella spp. $( \pm S E)$, from the Wellington Harbour and the South Coast, which came into contact with the mussel.

Of those Patiriella spp. that did contact the offered mussel, there was no significant difference in time taken to do so between Patiriella spp. from Wellington Harbour and Patiriella spp. from the Wellington South Coast $\left(F_{1,20}=0.06, p=0.82\right)$. The average time to contact was less than 30 minutes for both regions.

There was a non-significant difference in the time spent on the offered mussel between Patiriella spp. from Wellington Harbour and Patiriella spp. from the Wellington South Coast $(\mathrm{z}=1.81, \mathrm{p}=0.07)$. There was, however, a 
trend towards Patiriella spp. from the Harbour spending less time on the mussel than those from the South Coast (Figure 3.11).

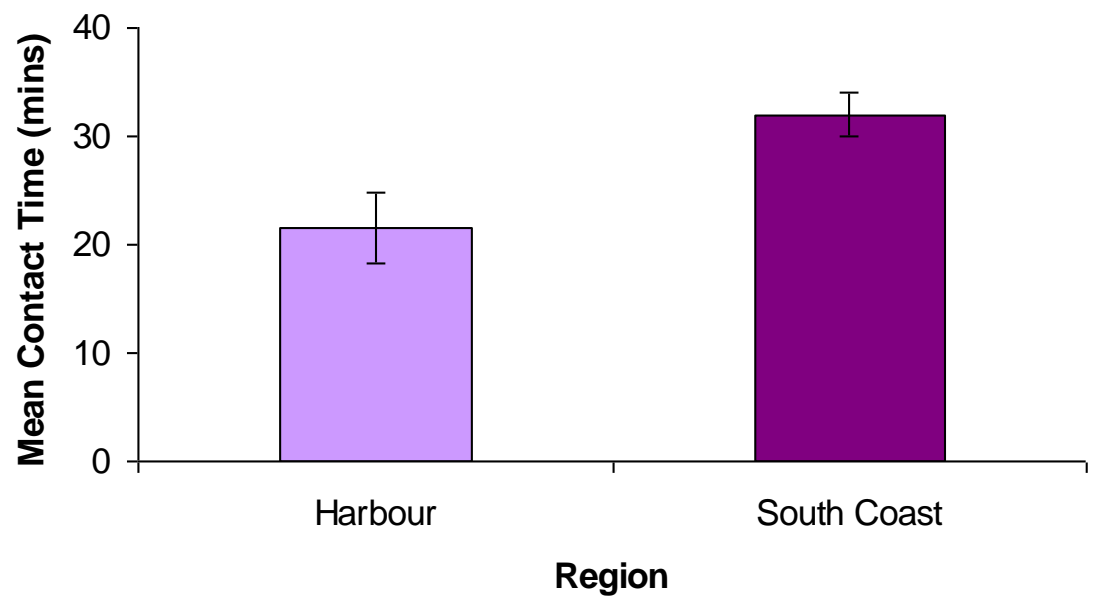

Figure 3.11: Mean time that Patiriella spp. from Wellington Harbour and Wellington South Coast stayed in contact with the mussel after first contact $( \pm S E)$. Excludes Patiriella spp. that did not contact the mussel at all.

\subsection{Discussion}

\section{Scavengers in the Field}

Patiriella spp. were attracted to mussel carrion, with multiple individuals found at the majority of bait stations, both in Wellington Harbour and on Wellington South Coast. This suggests that Patiriella spp. use distance chemoreception to detect and move towards carrion. Previous studies have debated the use of chemoreception by asteroids, possibly due to their behavioural variability (Sloan \& Northway, 1982). However, there is plenty of evidence for chemodetection in multiple seastar species (Sloan \& Northway, 1982; Rochette et al., 1994; Brewer \& Konar, 2005) and this appears to play an important role in their distribution and movement (Savy, 1987; Kurihara, 1999). The distribution of Patiriella spp. could be related to food resources, as they detect and move towards odours given off by carrion. However, there were bait stations that attracted none, or very few Patiriella spp., which suggests their distance for chemodetection is limited.

Previous studies have shown that the distance over which animals can detect odour is considerably variable among species. For example, the gastropod 
Nassarius dorsatus detects and responds to odours from a distance of $26 \mathrm{~m}$ (Morton \& Britton, 2003), whereas the smaller Ergalatax contractus can only detect odours from a maximum distance of $80 \mathrm{~cm}$ (Morton, 2006). Generally, the distance for chemodetection by intertidal and subtidal species is not as great as that for deep-sea scavengers (Sainte-Marie \& Hargrave, 1987). Scavengers that are able to detect carrion at a greater distance have the advantage, as they are more likely to reach a food source (Brewer \& Konar, 2005). Some of the bait stations that had few or no Patiriella spp. arrive also had low numbers of arriving whelks. Therefore, the low numbers of Patiriella spp. at these stations could have been caused by a limited or obstructed water flow, which reduced the spread of odour. However, limited distance chemodetection cannot be ruled out and arrival at the bait station is likely to be affected by a combination of odour spread and chemodetection abilities.

There was no significant difference between the numbers of Patiriella spp. attracted to bait in Wellington Harbour compared with those attracted on the Wellington South Coast, with an average of seven seastars per bait station in the Harbour and six on the South Coast. The most abundant species attracted to the bait was the red-mouthed whelk Cominella virgata, with an average density per bait station of 118 in the Harbour and 76 on the South Coast. The spotted whelk Cominella maculosa had similar densities to Patiriella spp. and the only other species that was consistently present at all bait stations was the big-handed crab Heterozius rotundifrons. There was no relationship between the numbers of whelks and the numbers of Patiriella spp. at bait stations. Therefore, there is no evidence of interspecific avoidance between these taxa. There could however, be competition for carrion food resources because it is likely to be in limited supply. Persson (1985) theorised that larger animals were likely to be superior at interference competition, but that smaller animals would be superior at exploitative competition, as they require fewer resources. In this study, the whelks present were the smaller competitor, but were also numerically dominant and so were likely to be superior at exploitation competition, due to their overall mass as well as their smaller individual requirements for energy resources. Similarly, the gastropod Nassarius festivus was found to be a competitively 
dominant scavenger over the hermit crab Diogenes edwardsii, due to its numerical superiority, as well as its ability for long distance chemodetection (Morton \& Yuen, 2000).

\section{Unfed Versus Supplemented}

The week-long feeding regime did have an effect on the weight gain of Patiriella spp., with supplemented seastars gaining significantly more weight than those that were either unfed or supplied only with cobbles. Patiriella spp. in both unfed and non-supplemented regimes lost weight. This is consistent with a 44-week study by Crump (1971), which found that Patiriella regularis supplemented with crabs or fish gained significant weight, while those deprived of macroscopic food lost weight. The gastropod Nassarius dorsatus also showed significant weight gain when fed on a supplemented diet (McKillup \& McKillup, 1997).

Significantly fewer of the supplemented Patiriella spp. came into contact with the mussel carrion offered, compared with those in unfed and nonsupplemented treatments. Patiriella spp. in unfed and non-supplemented treatments were also more likely to be feeding on the mussel than those previously supplemented with carrion. These results suggest that seastars that are hungrier are more likely to arrive at carrion and also more likely to feed on it. Fed asteroids often behave erratically, but starved individuals move more predictably in search of food (Rochette et al., 1994). This could be because the state of hunger of scavengers may affect their decision on whether or not to seek food (Morton \& Chan, 1999); therefore, numbers attracted to bait may be an indication of the hunger level of seastars as appears to be confirmed by these results. Other factors, such as population size, current direction (Morton \& Britton, 2003) and prey preference (Moore \& Lepper, 1997) may also affect the arrival of scavengers at bait, but these factors were all controlled within this experiment. Feeding in some seastars increases in the presence of conspecifics (Gaymer et al., 2002) and therefore overall numbers feeding may have been greater if multiple Patiriella spp. were tested at a time. 
The time taken for Patiriella spp. to arrive at the offered mussel and the length of time that the Patiriella spp. remained in contact was not significantly different among week-long feeding regimes. This suggests that while the hunger of an individual affects the decision to move towards and feed on carrion, it does not affect the speed of arrival or the length of time that the seastar remains on the carrion. This is consistent with a study by Morton and Chan (1999), that showed hunger affected whether or not the gastropod Nassarius siquijorensis arrived at food, but not the speed of arrival. Therefore, time taken for a Patiriella spp. to arrive is unlikely to be a good indication of an individual's hunger. The effect of starvation on the decision to arrive and the time to arrival appears to differ among species. Starvation affects the decision to move to carrion for $N$. siquijorensis (Morton \& Chan, 1999) and possibly Patiriella spp. Yet, movement towards carrion is not dependent on starvation for $E$. contractus, but instead the time this gastropod takes to arrive at the carrion is affected (Morton, 2006). However, conclusions taken from arrival time and contact time in this study are limited as the actual numbers arriving were low, leading to small sample sizes. There was a trend towards a longer arrival time and shorter contact time in previously supplemented seastars. However, further testing is required to conclusively determine whether previously starved individuals stay on prey longer due to reach satiation. In contrast, Morton found that starved gastropods often reach satiation faster (Morton, 2006). Alternatively, unfed seastars may require a longer feeding time as ingestion is slowed due to physiological damage (Stenton-Dozey et al., 1995), although this is unlikely to be the case here as Patiriella spp. were only starved for one week.

\section{Harbour Versus South Coast}

There was no significant difference in the likelihood of arrival to carrion, or the proportion of individuals feeding between Patiriella spp. from Wellington Harbour and Wellington South Coast. Feeding in the field prior to collection was not recorded, and this may have had an impact on the responses of Patiriella spp. to carrion. However, these results do indicate a contrast with 
week-long feeding regimes, in which arrival at bait and feeding appeared to be significant indicators of starvation. This suggests that there is no difference in the hunger levels of Patiriella spp. between regions and perhaps food resources are similar. It is interesting to note that, while not significant, there was a trend towards less Patiriella spp. from the South Coast arriving at the offered mussel. It is possible that Patiriella spp. from the South Coast had a lesser response to the carrion as mussels are not present on Wellington's South Coast (Gardner, 2000; Helson \& Gardner, 2004) and may not therefore be an easily recognised food resource. However, field experiments showed that Patiriella spp. were as readily attracted to mussel bait on Wellington's South Coast as they were in the Harbour. The proportions of Patiriella spp. from both the Harbour and the South Coast that were feeding, were similar to the proportions of unfed individuals feeding from the weeklong feeding experiment. Therefore, Patiriella spp. from the Wellington Harbour and the Wellington South Coast may both have a limited supply of carrion, affecting the hunger of animals from both regions. This is in agreement with the common view that carrion is a finite resource and therefore affects community structure (Morton, 2006).

Patiriella spp. from the Wellington Harbour and Wellington South Coast had similar arrival times at the mussel bait. This is consistent with results from the week-long feeding experiments, which showed the time to arrival was not significantly different at varying levels of hunger. The arrival time at bait is variable and ranges from approximately 5 to 60 minutes across all treatments. Therefore arrival time is not indicative of starvation or hunger in Patiriella spp.

The only significant result in comparison of Patiriella spp. from Wellington Harbour and Wellington South Coast, in relation to carrion attraction, was in the length of time that the seastars stayed in contact with the offered mussel. Those from the South Coast remained on the mussel bait for longer than individuals from the Harbour. This suggests that Patiriella spp. from the South Coast are hungrier, which appears to support our hypothesis that there is less food on the South Coast due to lower seston quality (Helson et al., 
2007). However, before making this conclusion the results from week-long feeding experiments must be examined to determine whether contact time is indicative of starvation. Although results from feeding experiments showed a trend towards longer contact time with greater starvation, these results were not significant and therefore inconclusive. Increased feeding times, and therefore length of time spent on carrion, have been linked to starvation (Stenton-Dozey et al., 1995), but for some organisms feeding rate and therefore feeding times are shorter when starved (Morton, 2006). Therefore, it is important to provide a reliable base pattern for each species before making assumptions about that species. Comparison of contact time with those from week-long feeding regimes shows that Patiriella spp. from Wellington Harbour and Wellington South Coast remain in contact with carrion close to the same time as those from unfed treatments. This suggests that scavenging Patiriella spp. from both the Wellington Harbour and the Wellington South Coast are hungry, due to limited food resources in both regions.

Limited food resources could mean that Patiriella spp. do not gain sufficient carrion to reach maturity and therefore these intertidal areas could be sink populations (Dias, 1996). However, seastars are mobile and therefore have the ability to move to areas with greater food resources. The seastar Marthasterias glacialis is an example of this, as it moves to shallow waters for feeding raids and then moves back to deeper waters (Savy, 1987). The distribution of seastars have also been shown to be affected by food resources, with distribution linked to food abundance in the seastar Asterina pectinifera (Kurihara, 1999). Attraction of Patiriella spp. to carrion, as shown in this study, suggests that their movement is likely to be affected by food resources. Therefore any changes in carrion supply, such as from fisheries discards, could potentially change the distribution of Patiriella spp. or enhance their population size (Ramsay et al., 1997b). These and other scavengers that can utilize discards may gain a competitive advantage, which can lead to an increase in numbers (Evans et al., 1996) and changes to community composition (Bozzano \& Sardà, 2002). Supply of carrion is erratic (Stenton-Dozey et al., 1995) and therefore, Patiriella spp. abundances 
could potentially be variable. This could mean instability in the community as it is continually changing, due to competition with changing Patiriella spp. populations.

\section{Summary}

Patiriella spp. are attracted to carrion, but only in limited numbers and this may be due to an ability to only use distance chemoreception over short distances. The numerically dominant scavenger in intertidal areas of the Wellington Harbour and Wellington South Coast was the red-mouthed whelk, Cominella virgata. Competition is possible between C. virgata and Patiriella spp., but there is no evidence of avoidance between these species.

Whether or not Patiriella spp. are feeding and whether or not they arrive at carrion, may be used as indicators of the hunger of individuals. Time taken for Patiriella spp. to arrive at carrion and time spent on the carrion does not appear to be affected by hunger. There is no evidence of a difference in starvation levels between Patiriella spp. from Wellington Harbour and Wellington South Coast. This suggests that carrion resources are similar between these regions. Food resources appear to be limiting for both regions, as results are more similar to those observed for unfed Patiriella spp. in week-long feeding regimes, than for those fed on an abundance of carrion. Variations in carrion resources have the potential to lead to instability in the community, due to changes in the abundances of Patiriella spp. and other scavenger species. 


\section{4}

\section{Inter- and Intraspecific Feeding Competition in Patiriella spp.}

\section{Abstract}

Competition can play an important role in structuring communities by affecting growth, survival and habitat range and aiding in food web stabilisation. Intraspecific competition often has a greater impact than interspecific competition, but the effects of both types of competition vary with species, space and time. This chapter examines intraspecific competition within Patiriella spp. and also interspecific competition between Patiriella spp. and a locally abundant chiton (Chiton glaucus). Laboratory experiments were conducted with varying densities of Patiriella spp. and C. glaucus to assess the impact of feeding competition on growth and mortality of each species, during spring and winter. Experimental treatments were supplied with food resources of crustose coralline algae and micro-organisms, with some intraspecific treatments additionally supplemented with mussel carrion. Experiments were run for six weeks, with supplemental experiments left to run for an additional four weeks. Density and type of competition (intra- or interspecific) appear to have no effect on the growth of either Patiriella spp. or C. glaucus, when not supplemented with carrion. The only nonsupplemented treatments to gain significant weight contained Patiriella spp. at low and medium densities in spring, with an average weight gain of just $4 \%$ for each treatment. By contrast, Patiriella spp. supplemented with mussel carrion exhibited significant weight gain at all densities, with an average weight gain of $45 \%$ at low densities, $51 \%$ at medium densities and $33 \%$ at 
high densities. Therefore, supplementation and density have a significant effect on Patiriella spp. growth. Supplementation also had a significant effect on the size of pyloric caeca, but not on gonad development. Mortality was low in all treatments. There appears to be no competition for crustose coralline algae or micro-organisms for either Patiriella spp. or C. glaucus, as this food resource may not be limited. However, there does appear to be intraspecific competition for carrion for Patiriella spp. and this is likely to have an impact in the field where this food resource in naturally limited.

\subsection{Introduction}

There has been much debate about the importance of competition in structuring communities. Schoener (1983) reviewed field experiments on interspecific competition and found that competition occurred in $90 \%$ of studies and $76 \%$ of species. A similar review by Connell (1983) concluded that $40 \%$ of studies and over half of species were affected by competition. While both reviews provide strong evidence that competition is a prevalent interaction, the variability between the reviews shows that the interpretation of competition experiments can be very subjective. Birch (1957) provided four definitions of competition as used in ecology, genetics and evolution. The strictest definition proposed by Birch states that competition occurs when a number of animals utilize common, limited resources; or when animals harm one another in an effort to gain access to a resource (Birch, 1957). Similarly, the current study defines competition as occurring when there is interaction over a shared resource that leads to negative effects for one or both of the individuals sharing the resource.

Competition can affect growth (Forrester et al., 2006), survival (Bonin et al., 2009) and habitat range (Connell, 1961) of individuals and populations, which may then have cascading effects on community structure. Additionally, competition acts in concert with abiotic factors and other biotic factors, such as predation. The interaction of competition and predation can act to stabilise food webs (Vandermeer, 2006). However, it is still important to investigate competition in isolation, in order to determine the extent of its role in shaping 
communities. Early studies have focussed on the role of interspecific competition in regulating communities, but more recently intraspecific competition has been recognised as playing a vital role in the distribution, abundance and resource use of species in natural communities (Connell, 1983), and is often found to have a greater effect than interspecific competition (Peterson, 1982; Forrester et al., 2006; Svanläck et al., 2008). The strength of competition is expected to increase with the level of overlap in resource use. Thus, as individuals of the same species are likely to overlap to a greater extent than individuals of different species, it is expected that intraspecific competition will be more intense. However, the overlap in resources does not always drive the strength of competition. For example, in reef fish communities Munday et al. (2001) found that some species with a seemingly large niche overlap do not compete and Bonin et al. (2009) found that interspecific competition was more important in post-settlement survival of coral reef fish. It appears that the relative importance of interspecific and intraspecific competition can vary with species and habitats. Therefore, it is important to test for both when attempting to assess the impact of competition on a community.

It has been suggested that competition may occur less frequently in small terrestrial animals due to their increased vulnerability to predation and physical disturbance compared to larger species (Schoener, 1983). By contrast, small terrestrial invertebrates that are hardy and resistant to predation, such as bees and ants, are often highly competitive. Persson (1985) suggested that smaller animals have the ability to be competitively superior at exploitation competition as they have a greater ability to withstand a reduction in resource availability. Seastars, while relatively small, are also hardy with few predators and therefore they have the potential to be strong competitors. Furthermore, competition is found to be more prevalent in marine systems than among species in terrestrial environments (Connell, 1983).

Field observations and experiments examining competition in seastars find different results depending on the species. For example, feeding competition 
between the predatory seastars Pisaster ochraceus and Leptasterias hexactis resulted in slower growth of the latter species (Menge, 1972). However, observations of Asterias forbesi and Asterias vulgaris found no evidence of competition, despite an overlap in resources (Menge, 1979). Strength of competition can also vary within the same species. Interference competition was found between the Australian Patiriella exigua species and the limpet Cellana tramoserica, with $P$. exigua retreating from a threatening C. tramoserica (Branch \& Branch, 1980). By contrast, Arrontes and Underwood (1991) were unable to find evidence of competition between these species, proposing that $P$. exigua avoids competition by moving to unexploited areas beyond the reach of gastropods. One explanation for the variation in findings among studies is that competition can be dependent on environmental factors (Gaymer et al., 2002), which can result in variable intensities of competition through time and space.

Branch and Branch (1980) also suggested that interference competition occurs between $P$. regularis and the limpet Cellana radians in New Zealand, but these species seldom co-occur in nature. Chitons, however, are abundant in cobble fields (Chapman, 2002b) and do co-occur with Patiriella spp. Chitons are generalist feeders and their diet is unclear, but appears to be similar to the herbivorous portion of Patiriella spp. diet (Crump, 1971), consisting mostly of algae and detritus (Creese, 1988). Chiton glaucus is the most abundant chiton species in Wellington Harbour and co-occurs with Patiriella spp. (see Chapter 2) and analysis of gut contents for this species has found high quantities of crustose coralline algae (Creese, 1988). These similarities in diet and distribution suggest possible competition between $C$. glaucus and Patiriella spp. for algae and micro-organisms.

Intraspecific competition was found in $P$. exigua, with densities negatively correlated to weight increase during caging experiments in the field (Branch \& Branch, 1980). Patiriella regularis have a similar diet to $P$. exigua, with both species appearing to feed on microalgae and possibly bacteria and detritus, as well as scavenging on carrion (Crump, 1971; Arrontes \& Underwood, 
1991). Therefore, intraspecific competition could be expected in Patiriella spp. at increased densities.

For most omnivorous scavengers carrion is important, with carrion availability affecting growth and reproduction (Morton \& Chan, 1999). Field experiments have found that the snail Nassarius dorsatus shows a significant growth increase when their diet is supplemented with fish, compared with nonsupplemented treatments (McKillup \& McKillup, 1997). Crump (1971) also found an increase in weight for $P$. regularis when they were supplemented on fish or crabs. The supply of carrion is often infrequent and unpredictable (Morton, 2006) and so competition for this scarce resource is likely to be an important factor for all scavengers. Patiriella regularis may compete with herbivores for algae, but as omnivorous scavengers (Crump, 1971) they also have the potential to compete for carrion with scavengers from other species or within their own species.

The aim of this chapter is to identify whether interspecific or intraspecific competition occurs in Patiriella spp., and whether intraspecific competition is mediated by the availability of carrion. The weight gain and mortality of Patiriella spp. and Chiton glaucus were examined at varying inter- and intraspecific densities. I hypothesised that: 1) for Patiriella spp. fed solely on algae and micro-organisms, increased density would have a negative effect on weight gain; 2) there would be a greater negative effect on weight gain for intraspecific treatments over interspecific treatments, due to greater intraspecific competition; 3) Patiriella spp. only treatments would have a greater negative effect on weight gain at high densities than low densities when supplemented on carrion; 4) supplemented Patiriella spp. would gain more weight than treatments fed only on algae and micro-organisms; 5) supplemented Patiriella spp. would have larger gonads than nonsupplemented Patiriella spp. because of greater weight gain; 6) weight gain would be greater in spring than in winter, due to an increase in feeding during warmer temperatures. 


\subsection{Methods}

\section{Growth Response to Competition}

\section{Experimental Setup}

Laboratory experiments were used to test the effects of intraspecific and interspecific competition on Patiriella spp. and Chiton glaucus (Figure 4.1). Varying densities of Patiriella spp. and C. glaucus were set up in baskets that measured $230 \mathrm{~mm}$ (width) $\times 300 \mathrm{~mm}$ (length) $\times 90 \mathrm{~mm}$ (height). Each basket was made up of a plastic frame, covered with shade cloth that allowed water and micro-organisms to pass freely in and out of the baskets, but restricted the seastars and chitons to their assigned basket. Each basket was labelled with a unique number and code to identify the individual basket and the treatment to which it belonged.

Cobbles were collected from Shelly Bay in Wellington Harbour and placed in the baskets at approximately the same density as found in the field (approximately 9 cobbles per basket). All cobbles were at least partially covered by crustose coralline algae as a possible source of food for Patiriella spp. and C. glaucus.

Baskets were placed in a seatable with a constant flow of unfiltered seawater that covered most of the cobbles. Inflow of seawater was at multiple locations across the seatable to minimise the bias of location of baskets within the table. Baskets were also placed randomly within the seatable and moved weekly. The height of the seawater was restricted to approximately $15 \mathrm{~mm}$ below the top edge of the baskets, as this was found to stop animals from escaping in trials. Unfiltered seawater was used as this was considered to be closest to field conditions and provided a continual supply of micro-organisms (i.e. another potential food source for both the seastars and the chitons).

The size and weight of the focal species was measured weekly over the duration of the experiments. To measure the wet weight of Patiriella spp. and chitons, individuals were first blotted to remove excess moisture before 
weighing. Patiriella spp. sizes were measured from the longest arm tip to the opposite armpit (see Figure 2.4) using callipers, whereas for chitons the length was measured. During experiments with $C$. glaucus as the focal individuals, only the size of chitons was measured on a weekly basis, as removing chitons from cobbles to weigh them would cause too much disturbance. Therefore chiton weight was only recorded at the beginning and end of each relevant experiment.

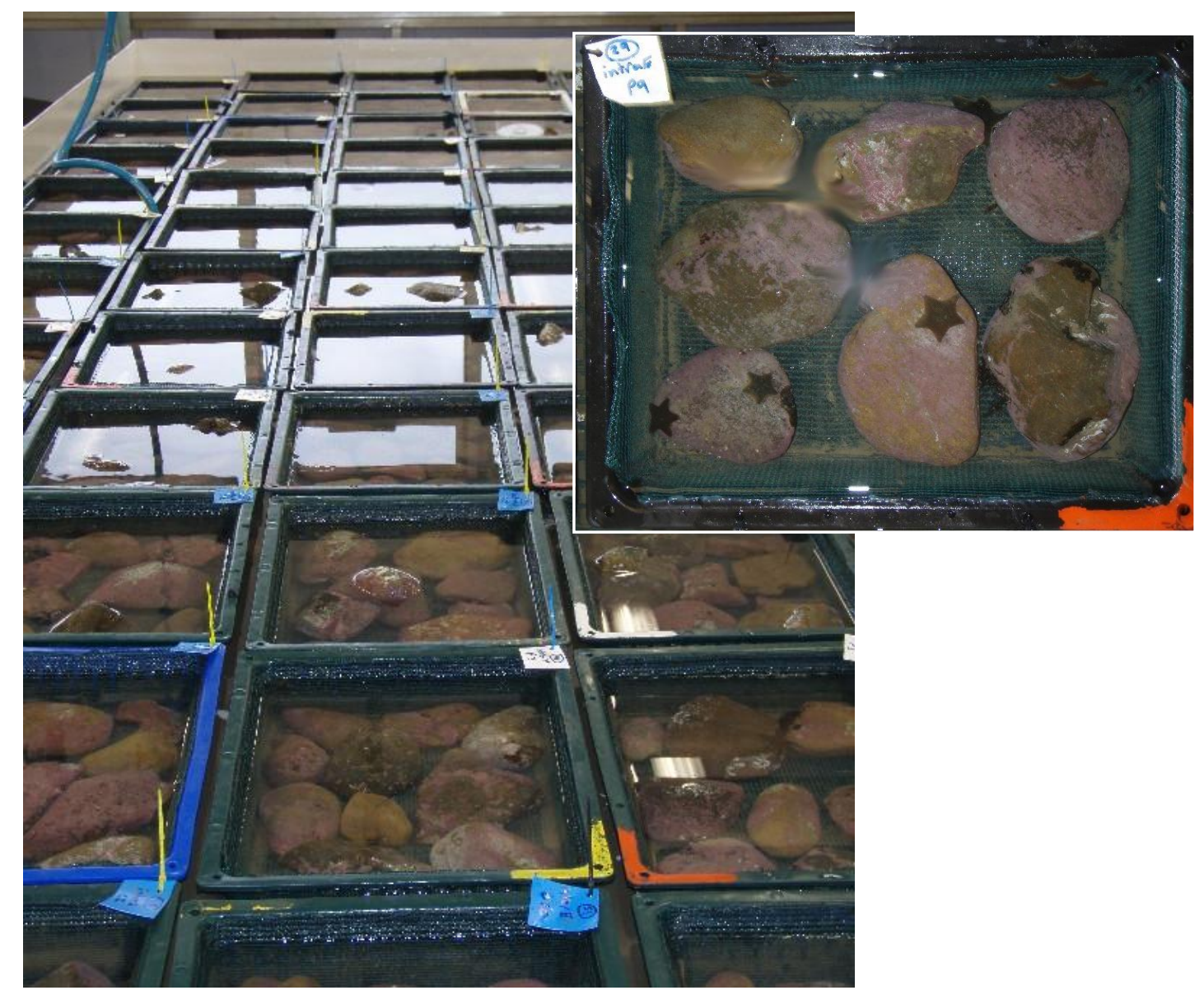

Figure 4.1: Experimental setup for growth and mortality response to competition.

\section{Treatments}

Six experiments were conducted to test whether competition affected growth or mortality (Table 4.1). At any one time two of these experiments were run concurrently. The first four experiments were run during winter, over June, July and August and each lasted six weeks. The first experiment examined the effect of intraspecific competition on Patiriella spp. by manipulating densities of this species. The second experiment (which was run 
concurrently) examined interspecific competition on Patiriella spp. in response to various densities of $C$. glaucus. These two experiments were then repeated as experiments three and four, with $C$. glaucus as the focal individual. C. glaucus growth was measured in the presence of varying densities of conspecifics and Patiriella spp. In all experiments there were ten duplicates of each treatment.

Experiments 5 and 6 were carried out over warmer months, during October, November and December, to examine the effects of temperature and food supplementation on competition and growth rates. These experiments were run for an extended period of ten weeks to examine whether growth patterns changed over time due to the seasonal reproductive cycle. Results from weeks one to six were compared with the previous experiments in winter. Experiment 5 again examined the effect of intraspecific competition on the growth of Patiriella spp. Concurrent to this, treatments were set up with the same densities of Patiriella spp., but this time they were supplemented with crushed mussels to examine the effect of supplementation on growth and intraspecific competition.

Table 4.1: Summary of treatments for competition experiments.

\begin{tabular}{cllcc}
\hline Experiment \# & Competition Type & Focal species & Supplemented & Season \\
\hline 1 & Intraspecific & Patiriella spp. & No & winter \\
2 & Interspecific & Patiriella spp. & No & winter \\
3 & Intraspecific & C. glaucus & No & winter \\
4 & Interspecific & C. glaucus & No & winter \\
5 & Intraspecific & Patiriella spp. & No & spring \\
6 & Intraspecific & Patiriella spp. & Yes & spring \\
\hline
\end{tabular}

\section{Densities}

The same low, medium and high densities were used in all experiments (Table 4.2). Low densities were based on the average densities per area, as recorded in the field, and medium densities were based on the maximum field densities. The high density of Patiriella spp. was three times higher than the maximum recorded in the field (Forrester et al., 2006) and the high density of $C$. glaucus was double the field maximum as it was not feasible to collect triple density of this species. 
Table 4.2: Summary of densities for competition experiments.

\begin{tabular}{|c|c|c|c|c|c|}
\hline Focal species & Competition type & Exp \#s & Density & Patiriella spp. \# & Chiton \# \\
\hline \multirow[t]{6}{*}{ Patiriella spp. } & Intraspecific & $1,5,6$ & Low & 1 & 0 \\
\hline & & & Medium & 3 & 0 \\
\hline & & & High & 9 & 0 \\
\hline & Interspecific & 2 & Low & 1 & 2 \\
\hline & & & Medium & 1 & 10 \\
\hline & & & High & 1 & 20 \\
\hline \multirow[t]{6}{*}{ C. glaucus } & Intraspecific & 3 & Low & 0 & 2 \\
\hline & & & Medium & 0 & 10 \\
\hline & & & High & 0 & 20 \\
\hline & Interspecific & 4 & Low & 1 & 2 \\
\hline & & & Medium & 3 & 2 \\
\hline & & & High & 9 & 2 \\
\hline
\end{tabular}

\section{Micro-organism Counts}

During six-week experiments where $C$. glaucus were the focal species (experiment 3 and 4), rough counts of micro-organisms were done to determine whether these organisms were available as a potential food source in the baskets. Every week clean microscope slides were placed in three random baskets. These always included at least one basket at each of high and low densities and at least one interspecific and one intraspecific treatment basket. At the end of each week, the microscope slides were examined at 400x magnification and all organisms were counted at three random positions on each slide.

\section{Reproductive Response to Competition}

At the conclusion of the final two experiments (experiments $5 \& 6$ ) one Patiriella spp. from each treatment was dissected to investigate reproductive provisioning. These seastars were initially frozen and then defrosted prior to dissection. Seastars were opened and the coelomic fluid was allowed to drain before the individual was blotted dry and weighed to give total body wet weight. All gonads were removed and drained of surface moisture before being weighed. The pyloric caeca were also removed and the remaining body was blotted and weighed to give the drained wet weight. The pyloric caeca were difficult to remove intact and so their weight was calculated by 
comparing the total body wet weight minus gonads and coelomic fluid to the drained wet weight.

To obtain organ indices the weight of gonads and pyloric caeca from all five rays was expressed as a percentage of the eviscerated body weight (Crump, 1971; Bos et al., 2008; Sanford et al., 2009):

(gonad weight / drained wet weight) ${ }^{*} 100$

(pyloric caeca weight / drained wet weight) ${ }^{*} 100$

Each individual was sexed according to gonad colour (Bos et al., 2008). Although variable, male gonads are generally creamy white and female gonads range from yellow to green (Crump, 1971). In some individuals the gonads were not visible or highly reduced and so sex could not be determined.

\section{Statistical Analysis}

Data were analysed using JMP 4.0.4. ANOVAs were run using EMS, but these results were compared to REML procedure and in all cases results were qualitatively the same. All figures show untransformed data for ease of interpretation. Homogeneity of variance was tested using Levene's test and Shapiro-Wilk's was used to test for normality for all ANOVA analysis.

All data from six-week feeding experiments was normally distributed. However, variances were not equal between intra- and interspecific Patiriella spp. treatments in winter. Variances were also unequal between supplemented and non-supplemented and density treatments in spring at both six and ten weeks, and among chiton density treatments. Log transformations did not correct for unequal variances and so ANOVA analysis continued with untransformed data. Non-parametric tests were not used, as they are not available for two-way ANOVA models. Gonad indices were not normally distributed, but variances were equal, so analysis was 
performed on untransformed data. Variances were unequal for pyloric caeca indices, which was corrected by log transforming the data.

Two-way ANOVAs were used to compare weight change over the course of the experiment. Separate ANOVAs were run to compare: 1) competition types in winter (inter- and intraspecific); 2) season (winter and spring); and 3) supplementation in spring (non-supplemented and supplemented). These analyses were not combined into one ANOVA as data was not available for all factors during all experiments and data would have been unbalanced. Competition type experiments with both Patiriella spp. and C. glaucus as the focal species used the following full model: competition type (fixed factor, 2 levels); density (fixed factor, 3 levels) and competition by density interaction. Season experiments with Patiriella spp. used the following model: season (fixed factor, 2 levels), density (fixed factor, 3 levels) and season by density interaction. Supplementation experiments for Patiriella spp. used the model: supplementation (fixed factor, 2 levels), density (fixed factor, 3 levels) and supplementation by density interaction. Post-hoc TUKEY tests were used to further compare treatments that had significant ANOVA results.

Paired t-tests were used to determine whether Patiriella spp. and C. glaucus had significantly different weights at the end of experiments compared to the start of experiments.

Two-way ANOVAs were also used to compare gonad indices and pyloric caeca indices, with a full model as follows: supplementation (fixed factor, 2 levels), density (fixed factor, 3 levels) and supplementation by density interaction, followed by TUKEY tests where appropriate. 


\subsection{Results}

\section{Growth Response to Competition}

\section{Non-supplemented Patiriella spp.}

There was no significant difference between intraspecific and interspecific treatments $\left(F_{1,54}=0.16, p=0.69\right)$ or densities $\left(F_{2,54}=0.22, p=0.80\right)$ on the weight change of non-supplemented Patiriella spp. during six-week feeding experiments in winter (Table 4.3; Figure 4.2). There were only four Patiriella spp. mortalities (3\%) during the six-week experiment and these all occurred in the first two weeks, across varying treatments.

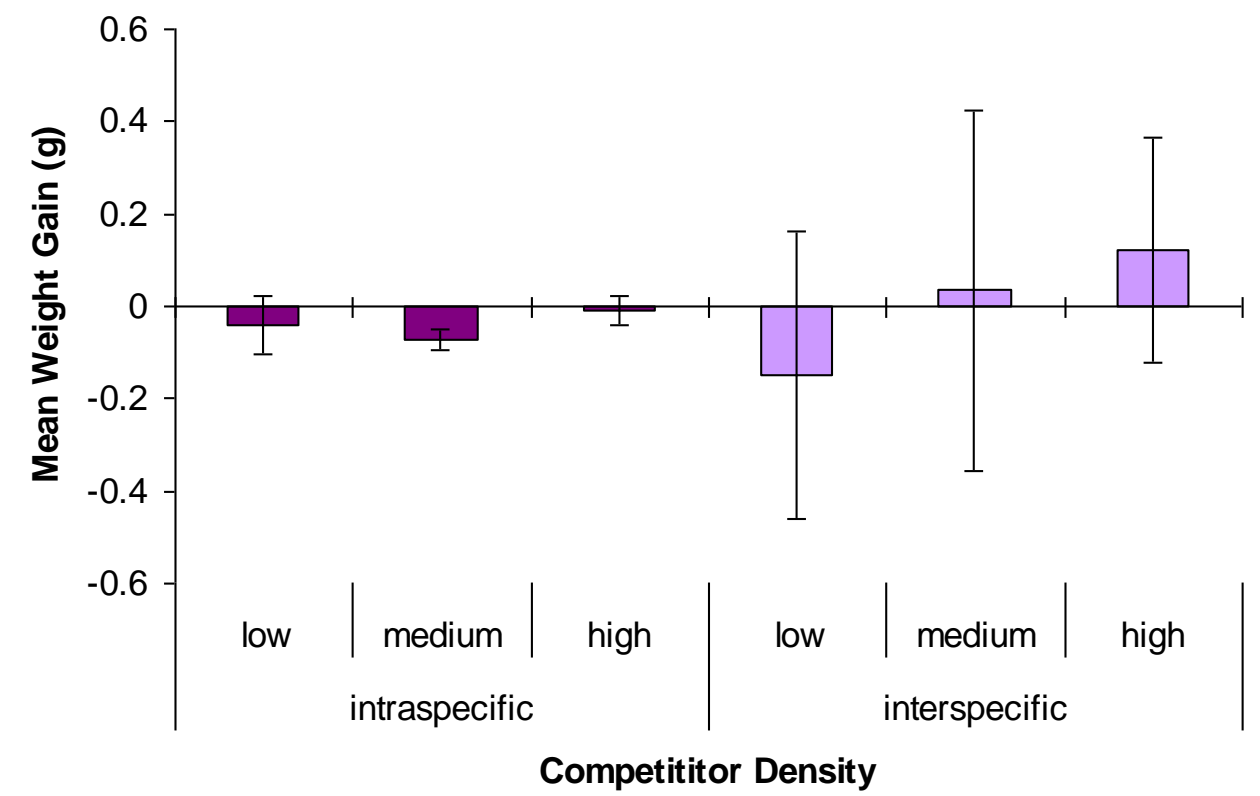

Figure 4.2: Mean weight change ( $\pm \mathrm{SE}$ ) of Patiriella spp. subjected to intra- and interspecific competition during six-week non-supplemented feeding experiments in winter.

The mean temperature of water used during winter laboratory experiments was $10^{\circ} \mathrm{C}$, with a mean water temperature of $13^{\circ} \mathrm{C}$ during spring experiments. During spring, Patiriella spp. in intraspecific treatments significantly gained weight at low $(\mathrm{t}=2.29, \mathrm{p}=0.05)$ and medium $(\mathrm{t}=2.81$, $\mathrm{p}=0.02)$ densities with an average weight gain of $0.12 \mathrm{~g}(4 \%)$ and $0.07 \mathrm{~g}(4 \%)$ respectively (Figure 4.3). During winter, however Patiriella spp. in intraspecific treatments at medium density significantly lost weight $(\mathrm{t}=-3.09$, $p=0.01$ ) with an average loss of $0.07 \mathrm{~g}(5 \%)$, and no change in weight over 
the course of the six weeks for the other two treatments. There was a significant interaction between the effects of season and density on the weight change of Patiriella spp. in intraspecific treatments $\left(F_{2,53}=3.34\right.$, $\mathrm{p}=0.04$; Table 4.3). A post-hoc TUKEY test showed that low densities in spring had a significantly greater weight change than medium densities in winter, with the latter losing weight. There was no significant difference among all other treatments. Weekly censuses of Patiriella spp. showed that an average of $29 \%$ were feeding at a single time during winter, with an average of $18 \%$ during spring.

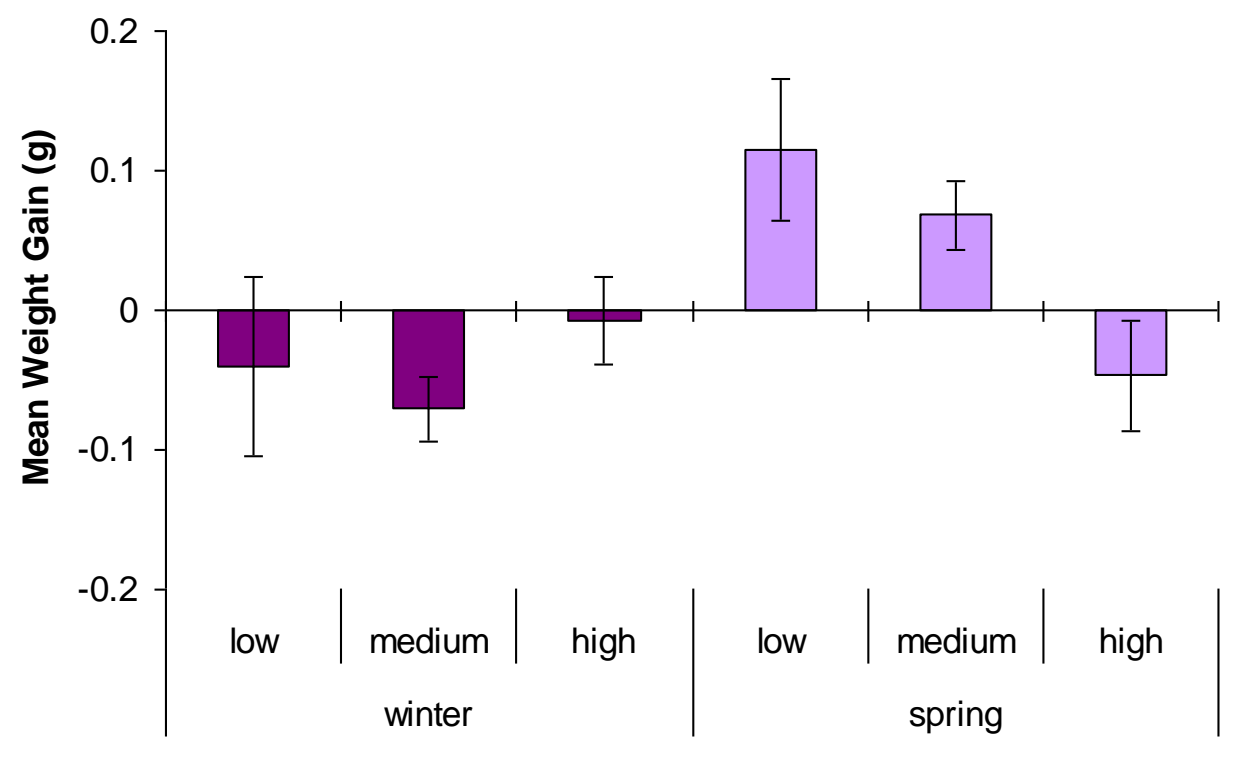

Competitor Density

Figure 4.3: Seasonal comparison of mean weight change ( $\pm \mathrm{SE})$ of Patiriella spp. during sixweek intraspecific feeding experiments where Patiriella spp. were not fed supplemental food.

\section{Supplemented Patiriella spp.}

All treatments during spring, except non-supplemented high densities, had a significantly greater end weight than start weight after six weeks (Figure 4.4). There was a significant effect of density $\left(F_{2,52}=7.88, \quad p<0.01\right)$ and supplementation $\left(F_{1,52}=25.94, p<0.0001\right)$ on weight change during spring experiments (Table 4.3). Post-hoc TUKEY analysis showed that the weight gain of supplemented seastars at all densities was significantly greater than the weight gain of non-supplemented seastars at all densities. Supplemented seastars at low densities also gained significantly more weight than supplemented seastars at both medium and high densities, with an average 
weight gain of $1.26 \mathrm{~g}(45 \%)$ at low densities, $0.94 \mathrm{~g}(51 \%)$ at medium densities and $0.69 \mathrm{~g}(33 \%)$ at high densities. Supplemented treatments at low densities almost always had mussel carrion remaining at the end of each week, whereas mussels in medium and high density treatments were often completely consumed each week. There was no significant difference in weight change between medium and high densities of supplemented seastars, nor any significant difference in weight change among any of the non-supplemented seastars. There was zero mortality of Patiriella spp. during the six-week feeding experiment during spring. Five small individuals did disappear from a single basket. This basket also contained relatively larger Patiriella spp. individuals that were present throughout the experiment, suggested possible cannibalism.

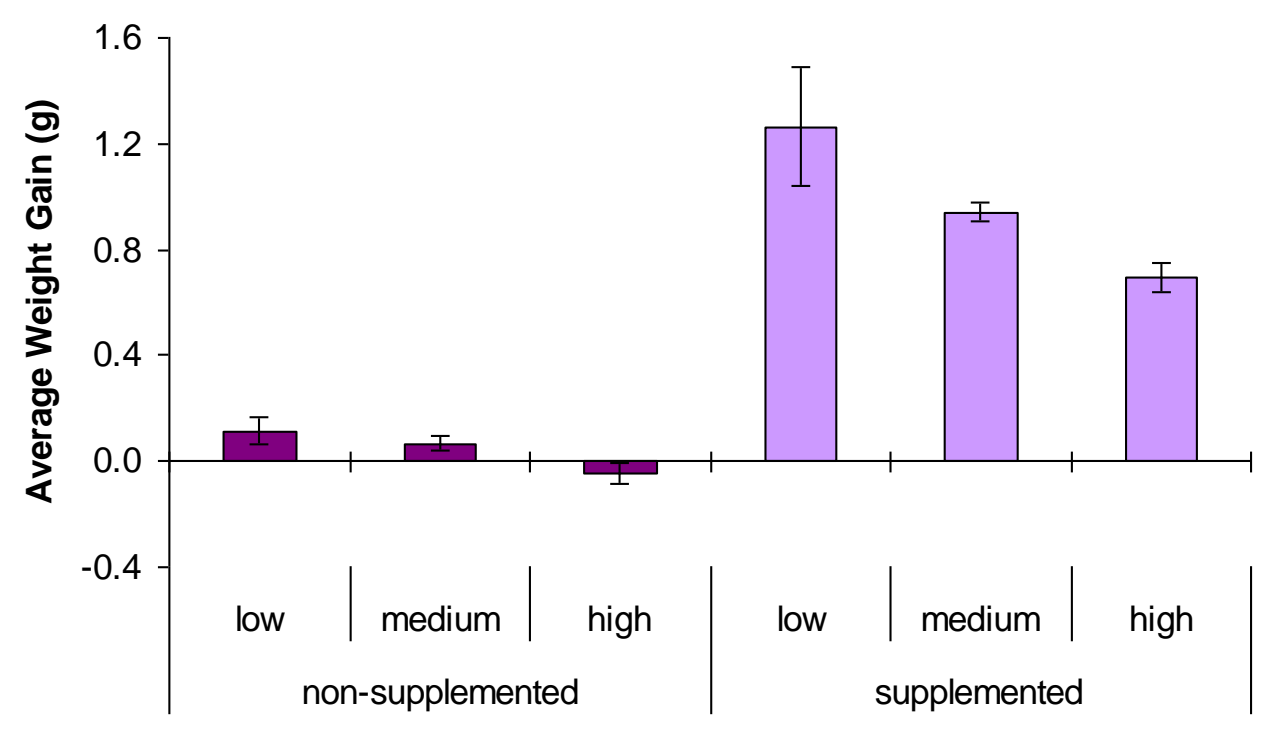

Competitor Density

Figure 4.4: Mean weight change ( \pm SE) of Patiriella spp. during six-week intraspecific feeding trials for supplemented and non-supplemented individuals in spring.

Supplemented Patiriella spp. showed a steady increase in weight during the first six weeks of the experiment, whereas the weight of non-supplemented Patiriella spp. stayed relatively stable. This pattern continued until week ten (Figure 4.5). 


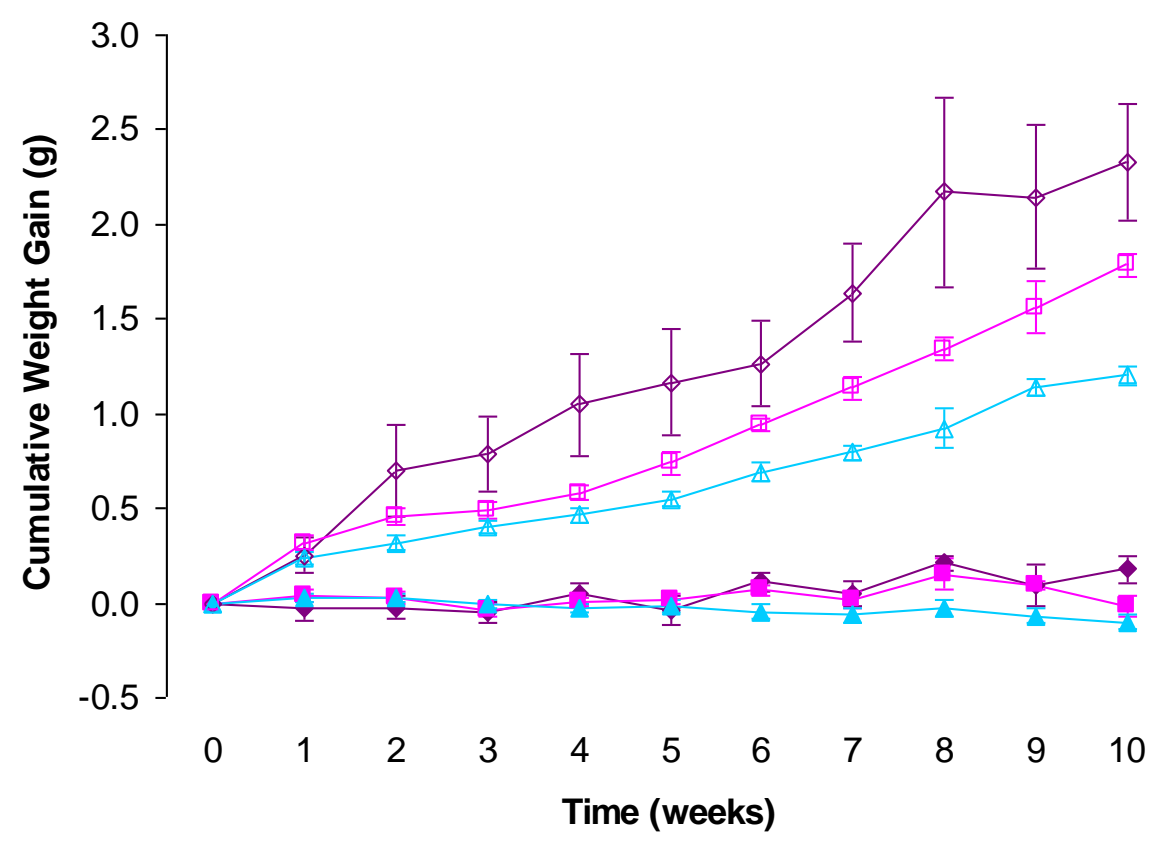

Figure 4.5: Mean cumulative weight change $( \pm S E)$ of Patiriella spp. individuals during tenweek feeding experiments in spring, showing weight change of supplemented Patiriella spp. (open symbols) and non-supplemented Patiriella spp. (solid symbols) at low densities (purple diamonds), medium densities (pink squares) and high densities (blue triangles).

Table 4.3: Results for ANOVAs investigating the change in weight of Patiriella spp.

\begin{tabular}{|c|c|c|c|c|c|}
\hline Model & Source of variation & $d f$ & MS & $\mathbf{F}$ & $p$ \\
\hline $\begin{array}{l}\text { Competition Type } \\
\text { (Non-supplemented } \\
\text { winter only) }\end{array}$ & $\begin{array}{l}\text { Competition type } \\
\text { Density } \\
\text { Competition type * density } \\
\text { Error }\end{array}$ & $\begin{array}{c}1 \\
2 \\
2 \\
54\end{array}$ & $\begin{array}{l}0.0837 \\
0.1150 \\
0.0855 \\
0.5224\end{array}$ & $\begin{array}{l}0.1602 \\
0.2202 \\
0.1638\end{array}$ & $\begin{array}{l}0.69 \\
0.80 \\
0.85\end{array}$ \\
\hline $\begin{array}{l}\text { Season } \\
\text { (Non-supplemented } \\
\text { intraspecific only) }\end{array}$ & $\begin{array}{l}\text { Season } \\
\text { Density } \\
\text { Season * density } \\
\text { Error }\end{array}$ & $\begin{array}{c}1 \\
2 \\
2 \\
53\end{array}$ & $\begin{array}{l}0.0075 \\
0.0206 \\
0.0564 \\
0.0169\end{array}$ & $\begin{array}{l}0.4472 \\
1.2206 \\
\mathbf{3 . 3 4 2 0}\end{array}$ & $\begin{array}{l}0.51 \\
0.30 \\
\mathbf{0 . 0 4}\end{array}$ \\
\hline $\begin{array}{l}\text { Supplementation } \\
\text { (Intraspecific spring } \\
\text { only) }\end{array}$ & $\begin{array}{l}\text { Supplementation } \\
\text { Density } \\
\text { Supplementation * density } \\
\text { Error }\end{array}$ & $\begin{array}{c}1 \\
2 \\
2 \\
52 \\
\end{array}$ & $\begin{array}{l}2.4709 \\
0.7503 \\
0.2959 \\
0.0953 \\
\end{array}$ & $\begin{array}{r}25.9392 \\
7.8766 \\
3.1059\end{array}$ & $\begin{array}{l}<0.0001 \\
<0.01 \\
0.05\end{array}$ \\
\hline
\end{tabular}

\section{Chiton glaucus}

Chiton glaucus in intraspecific treatments at medium ( $\mathrm{t}=-3.98, \mathrm{p}=0.01)$ and high $(\mathrm{t}=-4.74, \mathrm{p}<0.01)$ densities weighed significantly less after the six-week experiment during winter. There was no significant change in weight for all other C. glaucus treatments. There was also no significant interaction between density and type of competition (interspecific or intraspecific) in the 
weight gain of $C$. glaucus $\left(\mathrm{F}_{2,54}=0.07, \mathrm{p}=0.93\right)$, and no significant effect of competition type $\left(\mathrm{F}_{1,54}=1.43, \mathrm{p}=0.24\right)$ or density $\left(\mathrm{F}_{2,54}=2.61, \mathrm{p}=0.08\right.$; Figure 4.6). There were 16 C. glaucus mortalities (4\%) and these occurred across most treatments during the six-week experiment.

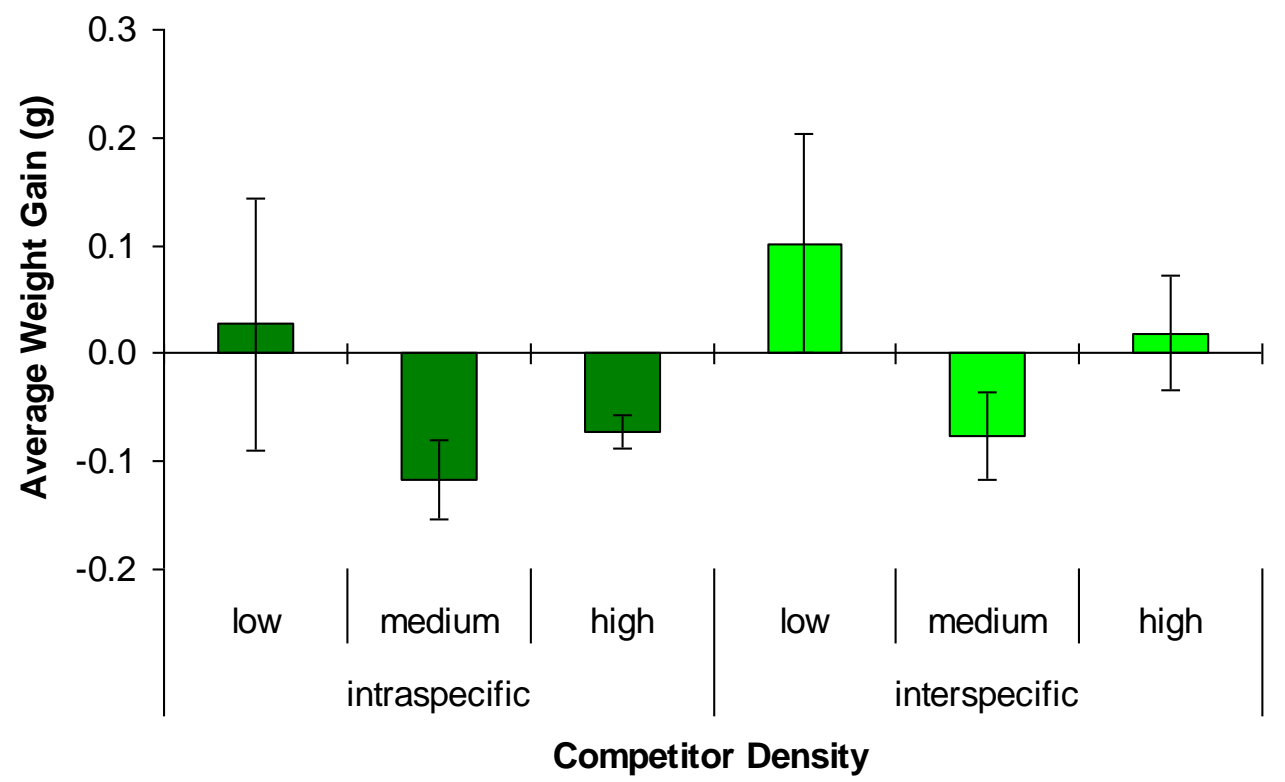

Figure 4.6: Mean weight change ( \pm SE) of Chiton glaucus subjected to intra- and interspecific competition during six-week feeding experiments.

Weekly counts of organisms settled on microscope slides showed that there was an abundance of micro-organisms present at all densities in baskets for both intra- and interspecific treatments. These organisms included various diatoms and other micro-algae, as well as occasional zooplankton. Crustose coralline algae, which was present on cobbles, was also observed as remaining alive throughout all experiments.

\section{Reproductive Response to Competition}

Each Patiriella spp. contained a pair of pyloric caeca running down each arm, which varied in colour from yellow, to orange to dark green. Pairs of gonads were positioned in the interradial space between each arm. There was no significant effect of supplementation $\left(F_{1,54}=2.02, p=0.14\right)$ or density $\left(F_{2}\right.$, ${ }_{54}=0.03, p=0.97$ ) on the gonad indices for Patiriella spp. after the ten-week experiment. Gonad indices varied considerably, with no pattern among treatments (Figure 4.7). 


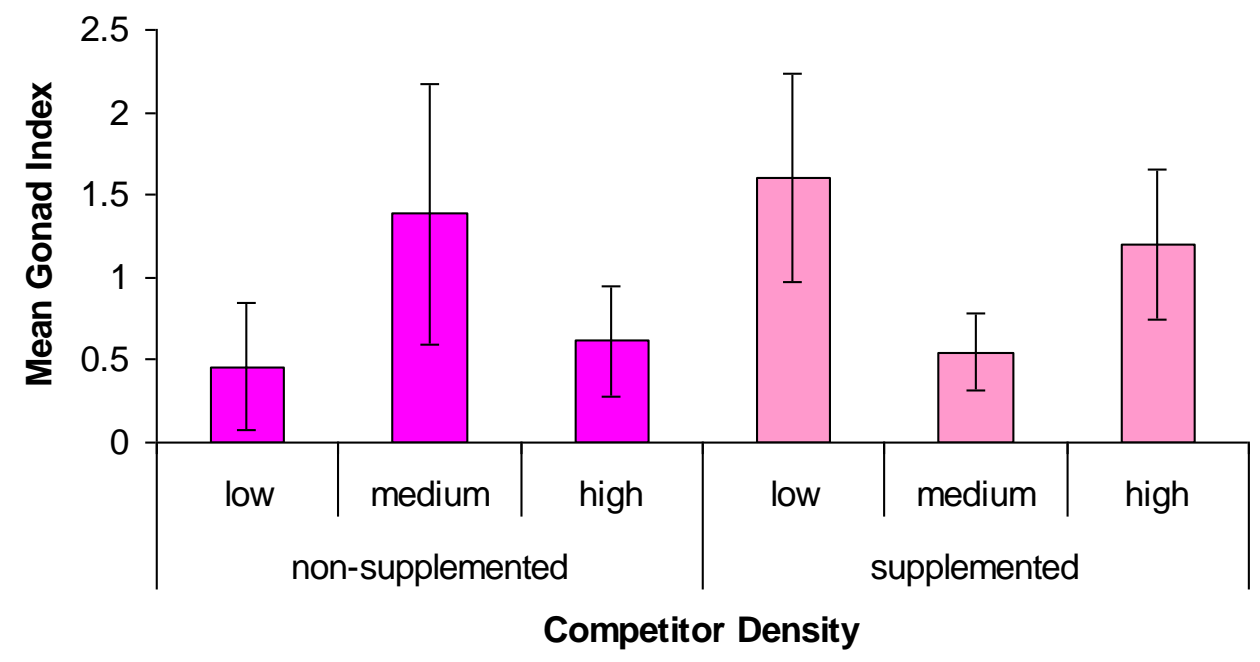

Figure 4.7: Mean gonad indices ( \pm SE) of Patiriella spp. after ten weeks at varying densities with and without supplementation.

There was a highly significant difference in the pyloric caeca indices between supplemented and non-supplemented Patiriella spp. $\left(F_{2,51}=17.84, p<0.001\right)$. Those Patiriella spp. that were supplemented on mussel had significantly larger pyloric caeca indices than those that fed only on algae and microscopic organisms (Figure 4.8). Density had no effect on pyloric caeca indices $\left(F_{2,51}=0.56, p=0.57\right)$ and there was no interaction between density and supplementation $\left(\mathrm{F}_{2,51}=0.75, \mathrm{p}=0.48\right)$.

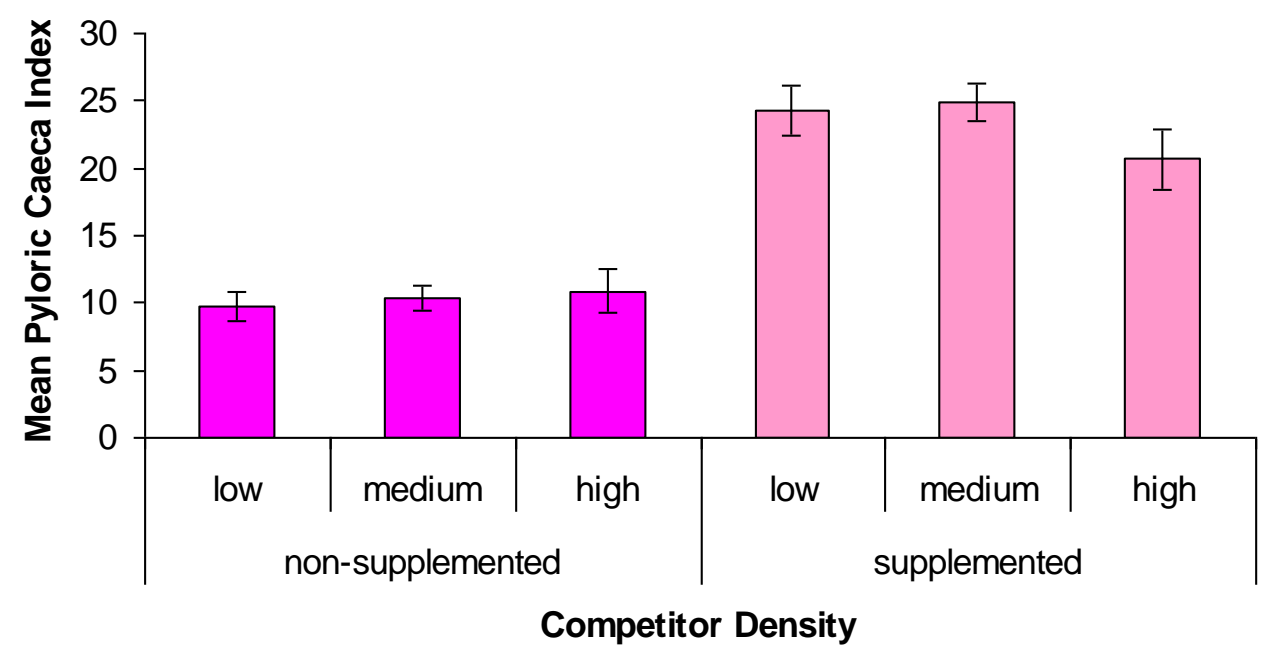

Figure 4.8: Mean pyloric caeca indices $( \pm S E)$ of Patiriella spp. after ten weeks at varying densities with and without supplementation. 


\subsection{Discussion}

\section{Growth Response of Non-supplemented Patiriella spp.}

Patiriella spp. that were only fed on crustose coralline algae and microorganisms showed very little overall change in weight for up to six weeks, with no difference among densities or between interspecific and intraspecific treatments. The growth of Patiriella spp. appears to be restricted during winter when only a diet of algae and micro-organisms is available. Competition for these resources does not appear to be the limiting factor, as growth did not occur even when only one Patiriella spp. was present and growth was not significantly different in treatments that contained greater densities of either Patiriella spp. or chitons.

There was a significant difference between growth of Patiriella spp. at medium densities in winter and low densities in spring. Low densities, which represent the average densities in the field, gained weight in spring and maximum field densities lost weight in the winter. This suggests that density and season have additive effects on growth. Feeding of other seastar species is temperature dependent. For example, in laboratory experiments, the predatory seastar Pisaster ochraceus consumed less prey at $9^{\circ} \mathrm{C}$ than at $12^{\circ} \mathrm{C}$ (Sanford, 1999). This is similar to the temperature differences in this study with winter temperatures of $10^{\circ} \mathrm{C}$ and spring temperatures of $13^{\circ} \mathrm{C}$. However, this temperature difference does not appear to have had a great effect on feeding or growth of Patiriella spp. By contrast, growth and survival of juvenile Patiriella pseudoexigua was found to vary significantly with changes in temperature and salinity (Chen \& Chen, 1993).

These results do not provide evidence of either inter- or intraspecific competition occurring for algae and micro-organisms. Given the abundance of micro-organisms and crustose coralline algae observed to be present during experiments, competition may not be occurring as these food resources are not limited. It is important to use caution when applying conclusions made from laboratory studies, but replication of these studies in the field is difficult due to problems isolating areas in three-dimensional 
cobble fields. A previous study with Patiriella exigua in the field also found problems with caging seastars (Branch \& Branch, 1980). Patiriella spp. can easily escape from cages and may also use extra surfaces provided by the side and top of a cage, which can confound results. Supply and growth of micro-organisms is likely to be even greater in the field, as water flow and conditions would increase the supply of algae and micro-organisms. Although access to algae and micro-organisms is not expected to be limited in the field, these food resources do not appear to provide sufficient nutrition to enable Patiriella spp. to gain weight. The limited weight loss and low mortality suggests that Patiriella spp., while not gaining weight, can still survive on this food source. Crump (1971) found that $P$. regularis, which were not fed macroscopic food, significantly lost weight during a 44-week laboratory study. Therefore, either the lack of weight loss in the current study is due to the shorter length of the experiment or the seastars were able to maintain their weight by feeding on crustose coralline algae. Burgett (1982) found that $P$. regularis were capable of decreasing the standing stocks of crustose coralline algae, which strongly suggests it is a food source. Algae also appear to be a major food source for other species of Patiriella. Patiriella pseudoexigua gained weight while fed a diet consisting only of benthic algae (Chen \& Chen, 1993) and escalated densities of $P$. exigua can inhibit development of algal cover (Arrontes \& Underwood, 1991). However, microorganisms cannot be excluded as a food source as $P$. regularis have been observed feeding on apparently bare rock (Crump, 1971) and the Australian species $P$. exigua leaves feeding scars in micro-algal biofilms (Jackson et al., 2009). Both crustose coralline algae and micro-organisms may be important for Patiriella spp. to survive during periods when other food resources are in short supply.

\section{Growth Response of Chiton glaucus}

Chiton glaucus maintained at average natural densities lost less weight than chitons at densities equivalent to the maximum observed in the field. Chitons held at densities representing field maxima showed significant weight loss during the six-week experiment. This suggests that competition may be 
occurring at maximum field densities and may be resulting in reduced growth when abundances are high. There was no difference between inter- and intraspecific competition on the growth of $C$. glaucus, which suggests that both forms of competition cause equivalent pressure. However, caution must be used when applying results from the laboratory to natural field populations. Two species of chiton, Acanthopleura brevispinosa and $A$. gemmata, were found to reduce interspecific competition by embarking on feeding excursions at different times (Chelazzi et al., 1983a), suggested that temporal partitioning may reduce competition. This type of avoidance was not possible for animals in the current study as they were unable to move far to feed. Therefore results seen here may be different to those that would occur in the field and should only be used as an indication of possible competition. Intraspecific competition has been observed in A. gemmata, with aggressive behaviour observed in the defence of homing indentations (Chelazzi et al., 1983b). When an individual intrudes on the homing position of another, the owner crawls over the intruder with its foot and tries to pull it out with fast back and forth movements. Where this aggression occurs, there is also depressed feeding from those chitons involved. This particular behaviour was not observed in the current study, but more than one individual often occupied the same cobble. Chitons did not appear to be homing during the laboratory experiment as the positions of chitons was variable, but $C$. glaucus have been noted as returning to the same position after night time excursions (Graham, 1941). Again this lack of homing could be an artefact of the laboratory experiments, as individuals no longer have access to what may have been their original homing positions.

Even at low densities, which contained only two chitons, there was no significant weight gain. Perhaps chiton growth is too slow to measure over six weeks and a longer experiment is required to effectively compare growth at varying densities. There are limited studies on the diet of chitons, but analysis of gut contents of $C$. glaucus found coralline algae, as well as diatoms, bacteria and cyanobacteria (Creese, 1988). It is unclear how much of this matter is digested and what passes through the gut undigested. However, at least some of these resources must form part of the chiton's 
diet. These resources were abundant during experiments and so lack of growth, especially at low densities cannot be attributed to food shortage. Alternatively chitons could be affected by laboratory conditions and stress, which led to restricted growth. Studies on other chiton species have found that they feed only during nocturnal low tides (Chelazzi et al., 1983a; Chelazzi et al., 1983b). All except a few large cobbles were submerged for the duration of laboratory experiments. Therefore, low tide was not simulated and chiton feeding may have been depressed, leading to their lack of growth. Growth rate may also decline with age (Otway, 1994) and therefore the expected growth is dependent on the age of chitons used for the experiment. The minimum longevity for chitons appears to be between six and eight years (Crozier, 1918; Otway, 1994). Age of chitons was not determined during this study and therefore it is not possible to determine if the lack of growth witnessed was similar to that which could be expected in the field.

\section{Growth Response of Supplemented Patiriella spp.}

Supplementing Patiriella spp. with carrion, in the form of a crushed mussel, significantly increased their weight gain. When carrion was present all density treatments steadily gained weight. Weight gain was significantly greater than the change in weight of non-supplemented treatments. This is consistent with a study by Crump (1971), which found that carrion supplementation was required for weight gain in $P$. regularis. The scavenger snail Nassarius dorsatus was also found to grow significantly faster with diet supplementation (McKillup \& McKillup, 1997).

Supplemented Patiriella spp. at high and medium densities gained less weight than those at low densities. Densities are representative of field densities, with medium densities similar to field maximums and low densities similar to average field density. For competition to occur the resource must be limiting (Birch, 1957). In laboratory experiments the provided mussel was completely consumed in the majority of medium and high-density treatments, implying that this resource was limited. Carrion is also a limited resource in the field (Morton, 2006) and is rarely naturally available in the intertidal 
(Britton \& Morton, 1994). Therefore intraspecific competition for carrion among Patiriella spp. in intertidal cobble fields is likely to occur, with a greater effect at maximum densities as shown by this study. In a study of scavenging hermit crabs Pagurus bernhardus, the intensity of competition increased when the density of competitors increased and the density of food resource decreased (Ramsay et al., 1997a). Morton and Yuen (2000) found that the hermit crab Diogenes edwardsii, which is a facultative scavenger, was less competitive than the obligate scavenger gastropod Nassarius festivus. It has therefore been suggested that obligate scavengers will be superior competitors (Morton \& Yuen, 2000) as they rely on an unpredictable carrion food resource (Britton \& Morton, 1994). Patiriella spp. have been observed feeding more frequently on algae and micro-organisms than on carrion (Crump, 1971), but this study shows that they also require carrion for growth and are therefore expected to be effective competitors for carrion.

In one treatment of this study all small Patiriella spp. disappeared within 5 weeks, and only two very large Patiriella spp. remained in the basket. There was no evidence of these missing individuals and escape was unlikely, as they were not found elsewhere. This treatment contained a high density of Patiriella spp. and was not supplemented with mussel carrion and therefore food was in short supply and seastars were likely to be hungry. Food deficiency in scavengers and opportunistic carnivores can often lead to cannibalism (Morton, 2006). The red land crab Gecarcinus lateralis preys on conspecific juveniles when food supply is poor quality (Wolcott \& Wolcott, 1984). A study of five marine scavengers found that likelihood of feeding on related species was increased by hunger (Moore \& Howarth, 1996). It is therefore possible that the large seastars consumed the small individuals, due to a shortage of food. However, it is unknown whether the small seastars were injured or dead prior to consumption.

\section{Reproductive Response of Patiriella spp.}

There was no significant effect of density or feeding treatment on the gonad indices of the Patiriella spp. However, the pyloric caeca of seastars 
supplemented on mussels were significantly larger than for those that were not supplemented. This is similar to the results for weight gain, which was also greater in supplemented seastars. However, the pyloric caeca indices were not significantly different among densities, which contrasts with body weight gain, which was greater at lower densities.

Gonad sizes were small overall, with only two individuals having gonad indices greater than 0.5. Gonad indices for the seastar Patiria miniata have been found to be poor indicators of reproductive state when they do not exceed 0.5 (Davis, 1985). In this study gonad size was not mediated by density or food availability, but these results may be affected by the overall small sizes of gonads. Crump (1971) found that after spawning the gonad indices of spent $P$. regularis were around 0.5 and that gonad indices were closer to 3.0 prior to spawning. A decrease in gonad size has been found to coincide with spawning events in various other species of seastars (Franz, 1986; Bos et al., 2008). Therefore, seastars in the current study may have already spawned. Conversely, gonads may be small because they have not yet become ripe. Spawning of $P$. regularis populations in Otago were observed to occur between December and February (Crump, 1971) and this study was concluded in early December. Therefore, it is likely that Patiriella spp. in this study had not yet spawned and that gonads may have become larger if experiments had continued through January and February.

Supplementation of Patiriella spp. appears to significantly increase the size of the pyloric caeca. The pyloric caeca indices of supplemented seastars were comparable to the indices previously recorded for laboratory fed $P$. regularis (Crump, 1971). The pyloric caeca of Patiriella spp. supplied with only microorganisms and crustose coralline algae were intermediate in size between those in a previous study that were starved and fed. Studies on other seastars have also found that pyloric caeca is related to food availability (Barker \& Xu, 1991; Barker \& Scheibling, 2008). This supports the previous findings in this chapter, that Patiriella spp. feed on micro-organisms and or algae, but they require carrion for growth. 
The pyloric caeca accumulates reserves during a sexual rest period, which are later utilised by the gonads during gametogenesis (Barker \& Xu, 1991). However, these reserves are also required for somatic maintenance and growth. It is expected a trade-off must exist between maintenance and reproduction when food resource are low (Grange et al., 2007). If this occurs then energy is concentrated on growth instead of gonad development (Harrold \& Pearse, 1980). Non-supplemented seastars had smaller pyloric caeca and showed no growth during ten-weeks in the laboratory. This suggests that they were receiving insufficient nutrition for growth, but perhaps enough for somatic maintenance. Therefore, it is unlikely that any energy would be focused on reproduction for these individuals if this food shortage continued. By contrast, supplemented seastars had relatively large pyloric caeca and steady growth, suggesting that the supplied mussel provided sufficient nutrition for maintenance and growth and perhaps also for reproduction during spawning periods. Repeated study of seastars at different reproductive stages throughout the year is required to make any definite conclusions about reproduction.

Pyloric caeca indices did not vary with density of supplemented Patiriella spp., which potentially indicates that they are at their maximum capacity for these individuals. When food is not limiting pyloric caeca sizes have been found to remain stable throughout the year (Menge, 1986; Grange et al., 2007). However, pyloric caeca indices have also been found to fluctuate inversely with gonad indices (Barker \& Xu, 1991; Byrne, 1992), due to transfer of energy to gonads prior to spawning (Crump, 1971). This inverse relationship varies with species (Byrne, 1992), time of year (Franz, 1986) and site (Menge, 1986).

\section{Summary}

Patiriella spp. supplied with only micro-organisms and crustose coralline algae in the laboratory were able to maintain weight and had low levels of mortality, but exhibited very little growth. Temperature and density appear to have a very small additive effect on growth of these non-supplemented 
seastars, but growth is still minimal even during spring at low densities. Therefore, nutrition supplied by micro-organisms and algae appears to be insufficient for Patiriella spp. growth and intraspecific and interspecific competition are not occurring for this food resource.

By contrast, Patiriella spp. supplemented with mussel carrion showed significant weight gain at all densities, with significantly more growth at lower densities. This indicates that Patiriella spp. require carrion for growth and that intraspecific competition is occurring for this food resource. As carrion is naturally limiting it is also likely that competition is occurring in the field. However, growth was still observed at all densities and so the implication of this competitive effect is unclear. Further research is required to determine the extent of competition in the field, which may depend on carrion availability.

Chiton glaucus individuals lost significant weight at maximum densities of conspecifics, but did not significantly lose weight at lower densities or at any density of Patiriella spp. This suggests that intraspecific competition may be occurring, resulting in reduced growth. However, these changes in weight were small and no significant weight gain was recorded at any density. Laboratory conditions and length of the study could have affected growth of C. glaucus and this requires further investigation.

Conclusions about reproductive potential are limited as gonad sizes were small for all treatments, possibly due to Patiriella spp. not being within their spawning period. Pyloric caeca indices were large for Patiriella spp. supplemented on mussel carrion. These pyloric caeca indices were constant across all densities suggesting the pyloric caeca were at their maximum size. Therefore, energy reserves are likely to be sufficient for gonad development during the spawning season for all densities of supplemented Patiriella spp. However, the pyloric caeca of non-supplemented Patiriella spp. were shrunken at all densities. This combined with the lack of growth implies that carrion supplementation is required for growth and reproduction of Patiriella spp. 


\section{5}

\section{General Discussion}

\subsection{Summary of Findings}

Patiriella spp. are abundant in intertidal cobble fields in New Zealand, yet little is know about their abundance, habits or ecological role, within these environments. The aim of this study was to increase the general knowledge of this seastar and to shed some light on its role and potential impact on the stability of the intertidal cobble community.

Field surveys provided baseline information on Patiriella spp. in their natural habitat among intertidal cobbles. Patiriella spp. were abundant within cobble fields both within Wellington Harbour and on the Wellington South Coast. Patiriella spp. appeared to be well dispersed, with most occupied cobbles containing only one individual. This is in contrast to studies which found that intertidal cobble inhabitants are generally over-dispersed, with many individuals on one cobble (Chapman, 2002b; 2005). Yet many cobbles contained no Patiriella spp. which, in-part, does fit the patterns found by Chapman (2002b; 2005). The reason for the distribution of animals within intertidal cobble fields is still unclear (McGuiness \& Underwood, 1986; Grayson \& Chapman, 2004; Chapman, 2005). Shade is an important cue for Patiriella regularis settlement (Byrne \& Barker, 1991), but this does not explain the distribution of mobile adults.

The distribution of adult Patiriella spp. could be related to carrion food availability. Only a small number of Patiriella spp. were found to be feeding on carrion in the field, but this could be related to its limited availability. Field 
and laboratory experiments showed that Patiriella spp. did actively move towards, and feed on, mussel carrion when available. Also, growth and storage only occurred for seastars that were fed carrion. A few individuals in the field were also seen feeding on parts of crab and abalone (Haliotis iris) carrion. Therefore, carrion is important to the diet of Patiriella spp. These findings are consistent with a study in Otago, which found that $P$. regularis feed predominantly on crustose coralline algae, but obtain the majority of their nutrition from occasional carrion (Crump, 1971). Due to the importance of carrion to the growth of Patiriella spp. and the active movement of the seastars towards provided mussel carrion, distribution is likely to be affected by this infrequent resource. Individuals may aggregate on carrion when it is available and then disperse when the resource is depleted.

Aggregation on carrion, which is limited in the field (Britton \& Morton, 1994), is likely to lead to intraspecific competition in Patiriella spp. Laboratory experiments showed that increasing the density of Patiriella spp. decreased the average weight gain of the seastars, when provided with mussel carrion. Growth was still observed at all densities, but this may not be the case in the field, where the carrion supply would not be as abundant. It appears that individuals that are unable to access carrion will not increase in size, will be unable to store excess energy in their pyloric caeca and are therefore unlikely to have enough reserves to reproduce. This suggests that intraspecific competition for carrion may play an important role in limiting the size and abundance of Patiriella spp.

No intraspecific response was observed when the seastars were only provided crustose coralline algae and micro-organisms. However, these food resources do not appear to be limiting, nor are they the main nutritional food source, as shown by this study and by Crump (1971).

Interspecific competition was proposed as another factor that may affect the distribution and abundance of Patiriella spp. within cobble fields. Chitons were observed in abundance in intertidal cobble habitats at all surveyed sites within Wellington Harbour and on the Wellington South Coast. A negative 
correlation was also found between the distribution of Patiriella spp. and chitons on cobbles. Chitons and Patiriella spp. appear to both feed on crustose coralline algae and/or micro-organisms (Creese, 1988) and so have the potential to compete for this resource. However, in laboratory experiments, no competition was found between the most common harbour chiton, Chiton glaucus, and Patiriella spp at all densities. Again this may be due to the abundance of the shared algal/micro-organism food resource.

The density of Patiriella spp. were similar between Wellington Harbour and the Wellington South Coast. However, there were marginal differences in Patiriella spp. sizes between region, with larger seastars on the South Coast compared with the Harbour. Patiriella spp were also feeding more frequently on the Wellington South Coast, than within the Harbour. The reasons for these differences are still unclear, but could be related to increased carrion availability or detection on the South Coast, or decreased competition for this resource. The South Coast has greater wave action, which may increase chemodetection (Gagnon et al., 2003), allowing Patiriella spp. to more easily detect and move towards carrion food resources. Carrion may also be more readily available in this region, due to deaths caused by wave action. The availability of carrion in both regions appears to be limited, with seastars from both regions reacting similarly to starved individuals, when presented with carrion in the laboratory. This suggests that intraspecific competition for carrion may be important in affecting the differences in sizes between the regions. As shown by controlled laboratory experiments, growth can be affected by intraspecific competition for carrion. This may be more pronounced in the field, where carrion is severely limiting (Britton \& Morton, 1994) and interspecific competition for carrion may also be occurring.

\subsection{Implications}

\section{Implications for Patiriella spp.}

Patiriella spp. growth was shown to be unaffected by the supply of crustose coralline algae or micro-organisms and the supply of this resource does not appear to be limiting. There was also no evidence of intraspecific competition 
for this food resource. Therefore the abundance of algae and microorganisms is unlikely to have a great effect on Patiriella spp. populations, assuming the supply remains unlimited.

Conversely, the supply of carrion did affect the growth of Patiriella spp. and there was intraspecific competition for this resource in laboratory experiments. Caution must be used when applying these laboratory results to natural populations in the field, but a correlation between density and size in the field can suggest that competition is occurring (Fletcher, 1984). Within this study, population sizes were similar at all sites and sizes varied, so there was no correlation. However, Crump (1971) found a negative correlation between density and size of $P$. regularis at sites in Otago. Therefore intraspecific competition may occur in the field.

Due to the effect of carrion on growth, the supply of carrion is likely to have an effect on Patiriella spp. field populations. An increase in the availability of carrion would increase the growth of individuals, which may lead to increased fecundity (Fletcher, 1984) and population size (Forrester et al., 2006). The population size of Patiriella spp. may therefore be regulated by carrion supply, as a subsequent decrease in carrion, would increase competition for the resource, restrict growth and eventually cause a decrease in population size.

Patiriella spp. appear to have few natural predators. Russell (1983) found a single $P$. regularis in the stomach of Porae (Nemadactylus douglasi), but suggested that $P$. regularis was an unimportant food source. Therefore bottom-up pressure, from carrion availability, is likely to be more important than top-down pressure from predation, in regulating population size.

\section{Implications for Direct Competitors}

This study found no evidence of feeding competition between Patiriella spp. and Chiton glaucus for crustose coralline algae and micro-organisms. Therefore Patiriella spp. are unlikely to have a direct effect on the growth and 
populations size of chitons in intertidal cobble fields and may also have little effect on other grazers in this habitat.

However, a change in the population size of Patiriella spp. may affect the local supply of crustose coralline algae, with flow on effects to intertidal grazers. There appeared to be no suppression of crustose coralline algae growth by Patiriella spp. in laboratory experiments. However, a study by Burgett (1982) found that increased densities of $P$. regularis were able to inhibit the growth of crustose coralline algae. The Australian species $P$. exigua has also been found to restrict the growth of crustose coralline algae (Arrontes \& Underwood, 1991; Jackson et al., 2009). By contrast, it has been suggested that $P$. exigua may enhance algal cover by only cropping, and not destroying, crustose coralline algae (Branch \& Branch, 1980). Grazing can enhance the growth of crustose coralline algae, as seen in grazing by the sea urchin Anthocidaris crassispina (Wai \& Williams, 2005). Therefore, grazing by Patiriella spp. in New Zealand may also have the same beneficial effect on crustose coralline algae. This may benefit other grazers in two ways: firstly, by increasing the abundance of crustose coralline algae; secondly, by keeping the algae cropped, as some grazers are only able to feed on cropped algae. Different grazers benefit at different levels of resource densities, due to their different feeding structures (Schmitt, 1996).

Intraspecific competition for carrion was observed for Patiriella spp. in the laboratory where the supplied mussel carrion was relatively plentiful, compared with the scarcity of carrion in the field (Britton \& Morton, 1994). Therefore, there may also be competition for this resource in the field between Patiriella spp. and other scavengers. This possible interspecific competition for carrion may have an effect on scavenger population dynamics.

\section{Implications for the Community}

Patiriella spp. feed most commonly on crustose coralline algae, but are thought to also consume newly settled invertebrates and are known to 
scavenge on carrion. Therefore, they may be considered omnivores with a strong potential to influence community dynamics. Omnivory stabilizes food webs by adding to complexity and dampening fluctuations (McCann \& Hastings, 1997; Coll \& Guershon, 2002). Patiriella spp. may therefore be important in structuring community dynamics. However, the impact of an omnivore on a food web is dependent on the strength of the omnivory (McCann \& Hastings, 1997). Patiriella spp. do feed at more than one trophic level. However, there is little evidence that they prey on live adult animals, except one report of them feeding on live mussels (Witman \& Grange, 1998). Therefore, their impact as omnivores on food web complexity may be weak.

As scavengers, Patiriella spp. play an important role in food webs by enhancing nutrient recycling (Selva \& Fortuna, 2007). However, changes in the ratio of scavenging to grazing may also affect trophic interactions (Petraitis, 2002). The greatest threat to community stability in relation to Patiriella spp. may be changes to carrion availability, such as discard of fishery bycatch (Ramsay et al., 1997b; Bozzano \& Sardà, 2002). Fishery bycatch can cause repeated or infrequent addition of carrion to the community, which may cause the density of scavengers, such as Patiriella spp. to increase or fluctuate. An increase in Patiriella spp. is then likely to increase interactions with other algal grazers and scavengers, leading to changes in food web stability and community dynamics.

\subsection{Future Directions}

Due to the limited time of this study, field surveys were only repeated twice within one year. While this information was useful in providing background for the current study, it can only accurately show details of Patiriella spp. populations in the Wellington Harbour and the Wellington South Coast at the time of the surveys. For a more complete understanding of the abundances, feeding habits and sizes of Patiriella spp. further surveys should be conducted. Repeated surveys at varying times of the year, across multiple years, would provide useful information about seasonal and yearly patterns and fluctuations. In this study month appeared to affect densities, but 
repeated monthly or seasonal surveys are required to make accurate predictions about this pattern. Weather conditions, and not time of year, may be a more important factor in affecting Patiriella spp. densities. Therefore, also repeating surveys during different weather conditions would be useful. Some of the patterns seen in this study varied from those seen by Crump (1971) during a study of Patiriella regularis in Otago. This could be due to differences in location, and so repeating surveys at different locations would also expand the knowledge of this understudied seastar.

Laboratory competition experiments were not repeated in the field due to difficulties with caging seastars and defining distinct areas within the threedimensional cobble habitat. If a method was found to accurately repeat experiments in the field, these results could more confidently be applied to natural populations. Tagging of Patiriella spp. was also not possible in this study. Successful tagging methods for this species would be useful in recording movements in the field. Further fieldwork to supplement this study may also include quantification of carrion supply within intertidal cobble fields in the Wellington Harbour and Wellington South Coast. While carrion is known to be a limiting resource (Britton \& Morton, 1994), an estimate of the actual carrion available within the study areas would improve understanding of the extent of this limitation and its effect on competitive interactions.

This study found an effect of intraspecific competition for carrion on the growth of Patiriella spp. However, interspecific competition was not tested for this food resource, but may be occurring in the field. Whelks were the most dominant taxa found on mussel bait in this study and so are possible competitors for carrion with Patiriella spp. Conducting interspecific competition experiments between Patiriella spp. and whelks may therefore be valuable. It is important to test for intra- and interspecific competition at the same time for an accurate comparison (Inouye, 2001). Therefore, intraspecific competition experiments for carrion should be repeated alongside the interspecific experiments. 
While Patiriella spp. have been observed feeding on crustose coralline algae, they also feed on what appears to be bare rock. It is still unclear whether they feed on algae, micro-organisms within the algae and on the bare rock, or a combination of these. Studies to date, including this one, have made assumptions about the diet of Patiriella spp. from the substrate upon which they are feeding. This has given us a good indication of their diet, but more thorough investigation is required. Previous studies have assumed either consumption of crustose coralline algae (Burgett, 1982) or micro-organisms (Crump, 1971). However, neither of these resources have been pinpointed as the actual source of the seastars nutrition, when not feeding on carrion. There is also debate about whether Patiriella spp. is a predator. Following field studies, Crump (1971) suggested that Patiriella spp. do not prey on live animals. However, Witman and Grange (1998) discussed the feeding of Patiriella spp. on live mussels. Clarification of Patiriella spp. diet is required to make further conclusions regarding their impact and ecological role.

\subsection{Conclusions}

Patiriella spp. are abundant in intertidal cobble fields in Wellington Harbour and on the Wellington South Coast. These seastars are seen feeding on crustose coralline algae and micro-organisms, but clarification of this diet is required. They also supplement their diet by feeding on carrion when it is available and require carrion for growth and possibly also reproduction.

Population sizes of Patiriella spp. were similar between Wellington Harbour and the Wellington South Coast. However, sizes of individuals were larger and feeding was more frequent on the South Coast than within the Harbour. This contradicts the initial hypothesis that sizes would be larger within the Wellington Harbour due to increased particulate food supply (Helson et al., 2007). However, these patterns could still be related to food supply, as carrion availability may be greater on the Wellington South Coast. Further investigation is required to determine the levels of carrion supply in both regions. Other factors such as preferred habitat or differences in larval supply or juvenile mortality may also be the cause of these size differences. 
Differences between Harbour and South Coast populations may also be related to vertical movement. For example, smaller Patiriella spp. may move to deeper waters on the South Coast to avoid harsher environmental conditions.

There is no evidence of either intra- or interspecific competition for crustose coralline algae or micro-organisms and this food resource does not appear to be limiting. Another study found that Patiriella regularis and the related species $P$. exigua had a negative impact on crustose coralline growth (Burgett, 1982; Arrontes \& Underwood, 1991) and others have reported positive impacts from other echinoderm grazers (Branch \& Branch, 1980; Wai \& Williams, 2005), but no such impacts were found by this study.

The supply of carrion appears to be important to the growth and reproduction of Patiriella spp., which has implications for size and abundance. The availability of carrion significantly increased the growth of individuals and the size of their pyloric caeca. Intraspecific competition for this resource was also observed, with reduced growth at increased densities. Carrion is know to be a scarce resource (Britton \& Morton, 1994) and Patiriella spp. from both Wellington Harbour and the Wellington South Coast behaved similarly to starved individuals when presented with mussel carrion in the laboratory. Also, very few Patiriella spp. were actually seen feeding on carrion in the field. However, when it is available Patiriella spp. actively move towards mussel carrion. This suggests that changes or fluctuations in carrion in the field, such as those caused by discarded bycatch, may have a significant impact on the distribution and population sizes of Patiriella spp. This may then impact other algal grazers and scavengers, leading to changes in food web stability and community dynamics. 


\section{References}

Arrontes, J. and Underwood, A. J. (1991) Experimental studies on some aspects of the feeding ecology of the intertidal starfish Patiriella exigua. Journal of Experimental Marine Biology and Ecology 148: 255269

Barker, M. F. and Russell, M. P. (2008) The distribution and behaviour of Patiriella mortenseni and $P$. regularis in the extreme hyposaline conditions of the Southern New Zealand Fiords. Journal of Experimental Marine Biology and Ecology 355: 76-84

Barker, M. F. and Xu, R. A. (1991) Population differences in gonad and pyloric caeca cycles of the New Zealand seastar Sclerasterias mollis (Echinodermata: Asteroidea). Marine Biology 108: 97-103

Barker, M. P. and Scheibling, R. E. (2008) Rates of fission, somatic growth and gonadal development of a fissiparous sea star, Allostichaster insignis, in New Zealand. Marine Biology 153: 815-824

Bertness, M. D. and Leonard, G. H. (1997) The role of positive interactions in communities: lessons from intertidal habitats. Ecology 78: 1976-1989

Bertness, M. D., Leonard, G. H., Levine, J. M., Schmidt, P. R. and Ingraham, A. O. (1999) Testing the relative contribution of positive and negative interaction in rocky intertidal communities. Ecology 80: 2711-2726

Birch, L. C. (1957) The meanings of competition. The American Naturalist 91: 5-18

Bonin, M. C., Srinivasan, M., Almany, G. R. and Jones, G. P. (2009) Interactive effects of interspecific competition and microhabitat on early post-settlement survival in a coral reef fish. Coral Reefs 28: 265274

Booth, J. D. (1975) Seasonal and tidal variations in the hydrology of Wellington Harbour. New Zealand Journal of Marine and Freshwater Research 9: 333-54

Bos, A. R., Gunamao, G. S., Alipooyo, J. C. E. and Cardona, L. T. (2008) Population dynamics, reproduction and growth of the Indo-Pacific horned sea star, Protereaster nodosus (Echinodermata; Asteroidea). Marine Biology 156: 55-63 
Bowman, M. J., Kibblewhite, A. C., Chiswell, S. M. and Murtagh, R. A. (1983) Shelf fronts and tidal stirring in greater Cook Strait, New Zealand. Oceanologica acta 6: 119-129

Bozzano, A. and Sardà, F. (2002) Fishery discard consumption rate and scavenging activity in the northwestern Mediterranean Sea. Journal of Marine Science 59: 15-28

Branch, G. M. and Branch, M. L. (1980) Competition between Cellana tramoserica (Sowerby) (Gastropoda) and Patiriella exigua (Lamarck) (Asteroidea) and their influence on algal standing stocks. Journal of Experimental Marine Biology and Ecology 48: 35-49

Brewer, R. and Konar, B. (2005) Chemosensory responses and foraging behavior of the seastar Pycnopodia helianthoides. Marine Biology 147: 789-795

Britton, J. C. and Morton, B. (1994) Marine carrion and scavengers. Oceanography and Marine Biology: An Annual Review 32: 369-434

Burgett, J. M. (1982). The feeding ecology of Patiriella regularis (Verrill) in the rocky intertidal. Zoology. Auckland, University of Auckland. MSc: 135.

Byrne, M. (1992) Reproduction of sympatric populations of Patiriella gunii, $P$. calcar and $P$. exigua in New South Wales, asterinid seastars with direct development. Marine Biology 114: 297-316

Byrne, M. (1996) Viviparity and intragonadal cannibalism in the diminutive sea stars Patiriella vivipara and P. parvivipara (family Asterinidae). Marine Biology 125: 551

Byrne, M. and Anderson, M. J. (1994) Hybridization of sympatric Patiriella species (Echinodermata: Asteroidea) in New South Wales. Evolution 48: $564-576$

Byrne, M. and Barker, M. F. (1991) Embryogenesis and larval development of the asteroid Patiriella regularis viewed by light and scanning electron microscopy. Biological Bulletin 180: 332-345

Byrne, M., Cisternas, P. and Koop, D. (2001) Evolution of larval form in the sea star genus Patiriella: conservation and change in the larval nervous system. Development, Growth and Differentiation 43: 459-468

Camus, P. A., Daroch, K. and Opazo, L. F. (2008) Potential for omnivory and apparent intraguild predation in rocky intertidal herbivore assemblages from northern Chile. Marine Ecology Progress Series 361: 35-45

Chapman, M. G. (2002a) Early colonization of shallow subtidal boulders in two habitats. Journal of Experimental Marine Biology and Ecology 275: 95-116 
Chapman, M. G. (2002b) Patterns of spatial and temporal variation of macrofauna under boulders in a sheltered boulder field. Austral Ecology 27: 211-228

Chapman, M. G. (2005) Molluscs and echinoderms under boulders: tests of generality of patterns of occurrence. Journal of Experimental Marine Biology and Ecology 325: 65-83

Chelazzi, G., Focaldi, S. and Deneubourg, J. L. (1983a) A comparative study on the movement of two sympatric tropical chitons (Mollusca: Polyplacophora). Marine Biology 74: 115-125

Chelazzi, G., Focardi, S., Deniubourg, J. L. and Innocenti, R. (1983b) Competition for the home and aggressive behaviour in the chiton Acanthopleura gemmata (Blainville) (Mollusca: Polyplacophora). Behavioural Ecology and Sociobiology 14: 15-20

Chen, B.-Y. and Chen, C.-P. (1992) Reproductive cycle, larval development, juvenile growth and population dynamics of Patiriella pseudoexigua (Echinodermata: Asteroidea) in Taiwan. Marine Biology 113: 271-280

Chen, C.-P. and Chen, B.-Y. (1993) The effect of temperature-salinity combinations on survival and growth of juvenile Patiriella pseudoexigua (Echinodermata: Asteroidea). Marine Biology 115: 119122

Coll, M. and Guershon, M. (2002) Omnivory in terrestrial arthropods: mixing plant and prey diets. Annual Review of Entomology 47: 267-297

Connell, J. H. (1961) The influence of interspecific competition and other factors on the distribution of the barnacle Chthamalus stellatus. Ecology 42: 710-723

Connell, J. H. (1972) Community interactions on marine rocky intertidal shores. Annual Review of Ecology and Systematics 3: 169-192

Connell, J. H. (1983) On the prevalence and relative importance of interspecific competition: evidence from field experiments. The American Naturalist 122: 661-696

Connolly, S. R. and Roughgarden, J. (1998) A latitudinal gradient in Northeast Pacific intertidal community strucutre: evidence for an oceanographically based synthesis of marine community theory. The American Naturalist 151: 311-325

Connolly, S. R. and Roughgarden, J. (1999) Theory of marine communities: competition, predation, and recruitment-dependent interaction strength. Ecological Monographs 69: 277-296 
Creese, R. G. (1988) Ecology of molluscan grazers and their interactions with marine algae in north-eastern New Zealand: a review. New Zealand Journal of Marine and Freshwater Research 22: 427-444

Crozier, W. J. (1918) Growth and duration of life of Chiton tuberculatus. Proceedings of the National Academy of Sciences 4: 322-325

Crump, R. G. (1971) Annual reproductive cycles in three geographically separated populations of Patiriella regularis (Verrill), a common New Zealand asteroid. Journal of Experimental Marine Biology and Ecology 7: $137-162$

Cruz-Motta, J. J. (2005) Diel and tidal variations of benthic assemblages in sediments associated with boulder fields. Marine Ecology Progress Series 290: 97-107

Curtis, L. A. and Hurd, L. E. (1979) On the broad nutritional requirements of the mud snail, Ilyanassa (Nassarius) obsoleta (Say), and its polytrophic role in the food web. Journal of Experimental Marine Biology and Ecology 41: 289-297

Davidson, I. C., Crook, A. C. and Barnes, D. K. A. (2004) Quantifying spatial patterns of intertidal biodiversity: Is movement Important? Marine Ecology 25: 15-34

Davis, K. K. (1985) DNA synthesis and the annual spermatogenic cycle in individuals of the sea star Patiria miniata. Biological Bulletin 169: 313327

Dayton, P. K. (1971) Competition, disturbance, and community organization: the provision and subsequent utilization of space in a rocky intertidal community. Ecological Monographs 41: 351-389

Demello, R. (2007). Processes influencing intertidal barnacle populations in the Wellington region. Wellington, Victoria University of Wellington. MSc: 114 .

Deygout, C., Gault, A., Sarrazin, F. and Bessa-Gomes, C. (2009) Modelling the impact of feeding stations on vulture scavenging service efficiency. Ecological Modelling 220: 1826-1835

Dias, P. C. (1996) Sources and sinks in population biology. Trends in Ecology and Evolution 11: 326-330

Evans, P. L., Kaiser, M. J. and Hughes, R. N. (1996) Behaviour and energetics of whelks, Buccinum undatum (L.), feeding on animals killed by beam trawling. Journal of Experimental Marine Biology and Ecology 197: 51-62 
Fletcher, W. J. (1984) Intraspecific variation in the population dynamics and growth of the limpet, Cellana tramoserica. Oecologia 63: 110-121

Forrester, G. E., Evans, B., Steele, M. A. and Vance, R. R. (2006) Assessing the magnitude of intra- and interspecific competition in two coral reef fishes. Oecologia 148: 632-640

Franz, D. R. (1986) Seasonal changes in pyloric caecum and gonad indices during the annual reporductive cycle in the seastar Asterias forbesi. Marine Biology 91: 553-560

Furness, R. W., Edwards, A. E. and Oro, D. (2007) Influence of management practices and of scavenging seabirds on availability of fisheries discards to benthic scavengers. Marine Ecology Progress Series 350: 235-244

Gagnon, P., Wagner, G. and Himmelman, J. H. (2003) Use of wave tank to study the effects of water motion and algal movement on the displacement of the sea star Asterias vulgaris towards prey. Marine Ecology Progress Series 258: 125-132

Gardner, J. P. A. (2000) Where are the mussels on Cook Strait (New Zealand) shores? Low seston quality as a possible factor limiting multi-species distributions. Marine Ecology Progress Series 194: 123132

Gaymer, C. F., Himmelman, J. H. and Johnson, L. E. (2002) Effect of intraand interspecific interactions on the feeding behaviour of two subtidal sea stars. Marine Ecology Progress Series 232: 149-162

Graham, D. H. (1941) Breeding habits of twenty-two species of marine mollusca. Transactions of the Royal Society of New Zealand 71: 152159

Grange, L. J., Tyler, P. A. and Peck, L. S. (2007) Multi-year observations on the gametogenic ecology of the Antarctic seastar Odontaster validus. Marine Biology 153: 15-23

Grayson, J. E. and Chapman, M. G. (2004) Patterns of distribution and abundance of chitons of the genus Ischnochiton in intertidal boulder fields. Animal Ecology 29: 363-373

Hairston, N. G., Smith, F. E. and Slobodkin, L. B. (1960) Community structure, population control, and competition. The American Naturalist 94: $421-425$

Harrold, C. and Pearse, J. S. (1980) Allocation of pyloric caecum reserves in red and starved sea stars, Pisaster giganteus (Stimpson): somatic maintenance comes before reproduction. Journal of Experimental Marine Biology and Ecology 48: 169-183 
Heath, R. A. (1971) Hydrology and circulation in central and southern Cook Strait, New Zealand. New Zealand Journal of Marine and Freshwater Research 5: 178-199

Heath, R. A. (1977) Circulation and hydrology of Wellington Harbour. New Zealand Oceanographic Summary 12:

Heath, R. A. (1985) A review of the physical oceanography of the seas around New Zealand - 1982. New Zealand Journal of Marine and Freshwater Research 19: 79-124

Heinrich, B. (1988) Winter foraging at carcasses by three sympatric corvids, with emphasis on recruitment by the raven, Corvus corax. Behavioural Ecology and Sociobiology 23: 141-156

Helson, J. G. and Gardner, J. P. A. (2004) Contrasting patterns of mussel abundance at neighbouring sites: does recruitment limitation explain the absence of mussels on Cook Strait (New Zealand) shores? Journal of Experimental Marine Biology and Ecology 312: 285-298

Helson, J. G., Pledger, S. and Gardner, J. P. A. (2007) Does differential particulate food supply explain the presence of mussels in Wellington Harbour (New Zealand) and their absence on neighbouring Cook Strait shores? Estuarine, Coastal and Shelf Science 72: 223-234

HilleRisLambers, R., Koppel, J. v. d. and Herman, P. M. J. (2006) Persistence despite omnivory: benthic communities and the discrepancy between theory and observation. Oikos 113: 23-32

Inouye, B. D. (2001) Response surface experimental designs for investigating interspecific competition. Ecology 82: 2696-2706

Jackson, A. C., Murphy, R. J. and Underwood, A. J. (2009) Patiriella exigua: grazing by a starfish in an overgrazed intertidal system. Marine Ecology Progress Series 376: 153-163

Keough, M. J. and Butler, A. J. (1979) The role of asteroid predators in the organization of a sessile community on pier pilings. Marine Biology 51: 167-177

Kurihara, T. (1999) Effects of sediment type and food abundance on the vertical distribution of the starfish Asterina pectinifera. Marine Ecology Progress Series 181: 269-277

Le Hir, M. and Hily, C. (2005) Macrofaunal diversity and habitat structure in intertidal boulder fields. Biodiversity and Conservation 14: 233-250

Maxwell, B. E. (1956) Hydrobiological observations for Wellington Harbour. Transactions of the Royal Society of New Zealand 83: 493-503 
McCann, K. and Hastings, A. (1997) Re-evaluating the omnivory-stability relationship in food webs. Proceedings of the Royal Society of London 264: $1249-1254$

McClintock, J. B., Angus, R. A., Ho, C., Amsler, C. D. and Baker, B. J. (2008) A laboratory study of behavioural interactions of the Antarctic keystone sea star Odontaster validus with three sympatric predatory sea stars. Marine Biology 154: 1077-1084

McClintock, J. B., Angus, R. A. and McClintock, F. E. (2007) Abundance, diversity and fidelity of macroinvertibrates sheltering beneath rocks during tidal emersion in an intertidal cobble field: Does the intermediate disturbance hypothesis hold for less exposed shores with smaller rocks? Journal of Experimental Marine Biology and Ecology 352: $351-360$

McGuiness, K. A. and Underwood, A. J. (1986) Habitat structure and the nature of communities on intertidal boulders. Journal of Experimental Marine Biology and Ecology 104: 97-123

McKillup, S. C. and McKillup, R. V. (1997) Effect of food supplementation on the growth of an intertidal scavenger. Marine Ecology Progress Series 148: $109-114$

Menge, B. A. (1972) Competition for food between two intertidal starfish species and its effect on body size and feeding. Ecology 53: 635

Menge, B. A. (1979) Coexistence between the seastars Asterias vulgaris and A. forbesi in a heterogenous environment: a non-equilibrium explanation. Oecologia 41: 245-272

Menge, B. A. (1986) A preliminary study of the reproductive ecology of the seastars Asterias vulgaris and A. forbesi in New England. Bulletin of Marine Science 39: 467-476

Menge, B. A. (1999) Top-down and bottom-up regulation of New Zealand rocky intertidal communities. Ecological Monographs 69: 297-330

Moore, P. A. and Lepper, D. M. E. (1997) Role of chemical signals in the orientation behavior of the sea star Asterias forbesi. Biological Bulletin 192: $410-417$

Moore, P. G. and Howarth, J. (1996) Foraging by marine scavengers: effects of relatedness, bait damage and hunger. Journal of Sea Research 36 : 267-273

Morton, B. (1986) Reproduction, juvenile growth, consumption and the effects of starvation upon the South China Sea whelk Hemisfusus tuba 
(Gmelin) (Prosobranchiata: Melonfenidae). Journal of Experimental Marine Biology and Ecology 102: 257-288

Morton, B. (1990) The physiology and feeding behaviour of two marine scavenging gastropods in Hong Kong: the subtidal Babylonia lutosa (Lamarck) and the intertidal Nassarius festivus (Powys). Journal of Molluscan Studies 56: 275-288

Morton, B. (2006) Scavenging behaviour by Ergalatax contraxtus (Gastropoda: Muricidae) and interactions with Nassarius nodifer (Gastropoda: Nassariidae) in the Cape d'Aguilar Marine Reserve, Hong Kong. Journal of the Marine Biological Association of the United Kingdom 86: 141-152

Morton, B. and Britton, J. C. (2003). The behaviour and feeding ecology of a suite of gastropod scavengers at Watering Cove, Burrup Peninsula, Western Australia. The Marine Flora and Fauna of Dampier, Western Australia. Western Australian Museum, Perth: 147-172.

Morton, B. and Chan, K. (1999) Hunger rapidly overrides the risk of predation in the subtidal scavenger Nassarius siquijorensis (Gastropoda: Nassariidae): and energy budget and a comparison with the intertidal Nassarius festivus in Hong Kong. Journal of Experimental Marine Biology and Ecology 240: 213-228

Morton, B. and Jones, D. S. (2003) The dietary preferences of a suite of carrion-scavenging gastropods (Nassariidae, Buccinidae) in Princess Royal Harbour, Albany, Western Australia. The Journal of Molluscan Studies 69: 151-156

Morton, B. and Yuen, W. Y. (2000) The feeding behaviour and competition for carrion between two sympatric scavengers on a sandy shore in Hong Kong: the gastropod, Nassarius festivus (Powys) and the hermit crab, Diogenes edwardsii (De Haan). Journal of Experimental Marine Biology and Ecology 246: 1-29

Morton, J. and Miller, M. (1968). The New Zealand Sea Shore. London, UK, Collins.

Munday, P. L., Jones, G. P. and Caley, M. J. (2001) Interspecific competition and coexistence in a guild of coral-dwelling fishes. Ecology 82: 21772189

Naylor, J. R. and McShane, P. E. (2001) Mortality of post-settlement abalone Haliotis iris caused by conspecific adults and wave exposure. New Zealand Journal of Marine and Freshwater Research 35: 363-369

O'Loughlin, P. M. and Waters, J. M. (2004) A molecular and morphological revision of genera of Asterinidae (Echinodermata: Asteroidea). Memoirs of Museum Victoria 61: 1-40 
O'Loughlin, P. M., Waters, J. M. and Roy, M. S. (2002) Description of a new species of Patiriella from New Zealand, and review of Patiriella regularis (Echinodermata, Asteroidea) based on morphological and molecular data. Journal of The Royal Society of New Zealand 32: 697711

Otway, N. M. (1994) Population ecology of the low-shore chitons Onithochiton quercinus and Plaxiphora albida. Marine Biology 121: 105-116

Paine, R. T. (1966) Food web complexity and species diversity. The American Naturalist 100: 65-75

Paine, R. T. (1971) A short-term experimental investigation of resource partitioning in a New Zealand rocky intertidal habitat. Ecology 52: 1096-1106

Paine, R. T. (1980) Food webs: linkages, interaction strength and community infrastructure. Journal of Animal Ecology 49: 667-685

Paine, R. T. and Vadas, R. L. (1969) The effects of grazing by sea urchins, Strongylocentrotus spp., on benthic algal populatioons. Limnology and Oceanography 14: 710-719

Palumbi, S. R. (1997) A star is born. Nature 390: 556-557

Persson, L. (1985) Asymmetrical competition: Are larger animals competitively superior. American Naturalist 126: 261-266

Peterson, C. H. (1982) The importance of predation and intra-and interspecific competition in the population biology of two infaunal suspension-feeding bivalves, Protothaca staminea and Chione undatella. Ecological Monographs 52: 437-475

Petraitis, P. S. (2002) Effects of intraspecific competition and scavenging on growth of the periwinkle Littorina littorea. Marine Ecology Progress Series 236: 179-187

Phillips, N. E. and Hutchison, E. (2008) Grazer effects on algal assemblages and mussel recruitment in two different mid-intertidal communities in the Cook Strait, New Zealand. New Zealand Journal of Marine and Freshwater Research 42: 297-306

Pimm, S. L. and Lawton, J. H. (1978) On feeding on more than one trophic level. Nature 275: $542-544$

Polis, G. A. and Strong, D. R. (1996) Food web complexity and community dynamics. The American Naturalist 147: 813-846 
Ramsay, K., Kaiser, M. J. and Hughes, R. N. (1997a) A field study of intraspecific competition for food in hermit crabs (Pagurus bernhardus). Estuarine, Coastal and Shelf Science 44: 213-220

Ramsay, K., Kaiser, M. J., Moore, P. G. and Hughes, R. N. (1997b) Consumption of fisheries discards by benthic scavengers: utilization of energy subsidies in different marine habitats. Journal of Animal Ecology 66: 884-896

Rochette, R., Hamel, J.-F. and Himmelman, J. H. (1994) Foraging strategy of the asteroid Leptasterias polaris: role of prey odors, current and feeding status. Marine Ecology Progress Series 106: 93-100

Russell, B. C. (1983) The food and feeding habits of rocky reef fish of northeastern New Zealand. New Zealand Journal of Marine and Freshwater Research 17: 121-145

Sainte-Marie, B. and Hargrave, B. T. (1987) Estimation of scavenger abundance and distance of attraction to bait. Marine Biology 94: 431443

San, E. D. L., Malongwe, N., Fike, B., Somers, M. and Walters, M. (2009) A diverse autumn diet without dominant prey for opportunistic blackbacked jackals Canis mesomelas. Wildlife Biology in Practise 5: 96103

Sanford, E. (1999) Regulation of keystone predation by small changes in ocean temperature. Science 283: 2095-2096

Sanford, E., Wood, M. E. and Nielson, D. J. (2009) A non-lethal method for estimation of gonad and pyloric caecum indices in sea stars. Invertebrate Biology 128: 372-380

Savy, S. (1987) Activity pattern of the sea-star Marthaserias glacialis, in PortCros Bay (France, Mediterranean Coast). Marine Ecology 8: 97-106

Schmitt, R. J. (1996) Exploitation competition in mobile grazers: trade-offs in use of a limited resource. Ecology 77: 408-425

Schoener, T. W. (1983) Field experiments on interspecific competition. The American Naturalist 122: 240-285

Selva, N. and Fortuna, M. A. (2007) The nested structure of a scavenger community. Proceedings of the Royal Society 274: 1101-1108

Shima, J. S. and Swearer, S. E. (2009a) Larval quality is shaped by matrix effects: implications for connectivity in a marine metapopulation. Ecology 90: 1255-1267 
Shima, J. S. and Swearer, S. E. (2009b) Spatially variable larval histories may shape recruitment of a temperate reef fish. Marine Ecology Progress Series 394: 223-229

Sloan, N. A. and Northway, S. M. (1982) Chemoreception by the asteroid Crossaster papposus (L.). Journal of Experimental Marine Biology and Ecology 61: 85-98

Smoothey, A. F. and Chapman, M. G. (2007) Small-scale variability in the dispersion of the sea urchin Heliocidaris erythrogramma among boulders. Marine Ecology Progress Series 340: 89-99

Sousa, W. P. (1979) Disturbance in marine intertidal boulder fields: the nonequilibrium maintenance of species diversity. Ecology 60: 12251239

Stenton-Dozey, J. M. E., Brown, A. C. and O'Riain, J. (1995) Effects of diet and starvation on feeding in the scavenging neogastropod Bullia digitalis (Dillwyn). Journal of Experimental Marine Biology and Ecology 186: $117-132$

Stevenson, J. P. (1992) A possible modification of the distribution of the intertidal seastar Patiriella exigua (Lamarck) (Echinodermata : Asteroidea) by Patiriella calcar (Lamarck). Journal of Experimental Marine Biology and Ecology 155: 41-54

Svanläck, R., Eklöv, P., Fransson, R. and Holmgren, K. (2008) Intraspecific competition drives multiple species resource polymorphism in fish communities. Oikos 117: 114-124

Turkington, R. (2009) Top-down and bottom-up forces in mammalian herbivore - vegetation systems: an essay review. Botany 87: 723-739

Underwood, A. J. (2000) Experimental ecology of rocky intertidal habitats: what are we learning? Journal of Experimental Marine Biology and Ecology 250: 51-76

Underwood, A. J., Chapman, M. G. and Connell, S. D. (2000) Observations in ecology: you can't make progress on processes without understanding the patterns. Journal of Experimental Marine Biology and Ecology 250: 97-115

Vandermeer, J. (2006) Omnivory and the stability of food webs. Journal of Theoretical Biology 238: 497-504

Wai, T.-C. and Williams, G. A. (2005) The relative importance of herbivoreinduced effects on productivity of crustose coralline algae: sea urchin grazing and nitrogen excretion. Journal of Experimental Marine Biology and Ecology 324: 141-156 
Waters, J. M. and Roy, M. S. (2004) Phylogeography of a high-dispersal New Zealand sea-star: does upwelling block gene-flow? Molecular Ecology 13: $2797-2806$

Wentworth, C. K. (1922) A scale of grade and class terms for clastic sediments. The Journal of Geology 30: 377-392

Witman, J. D. and Grange, K. R. (1998) Links between rain, salinity, and predation in a rocky subtidal community. Ecology 79: 2429-2447

Wolcott, D. L. and Wolcott, T. G. (1984) Food quality and cannibalism in the red land crab, Gecarcinus lateralis. Physiological Zoology 57: 318-324 